University of Redlands

\title{
Identifying and Viewing Rooftop Solar Potential: A Case Study for the City of Redlands, California
}

A Major Individual Project submitted in partial satisfaction of the requirements

for the degree of Master of Science in Geographic Information Systems

by

Taira Nakanishi

Fang Ren, Ph.D., Committee Chair

Mark Kumler, Ph.D.

August 2010 
Identifying and Viewing Rooftop Solar Potential:

A Case Study for the City of Redlands, California

Copyright (C) 2010

by

Taira Nakanishi 
The report of Taira Nakanishi is approved.

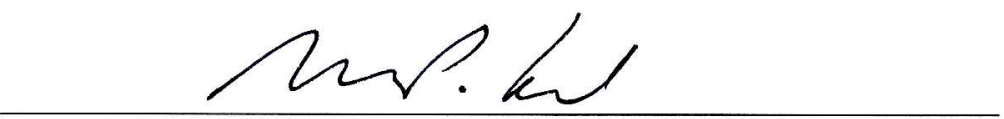

Mark Kumler, Ph.D.

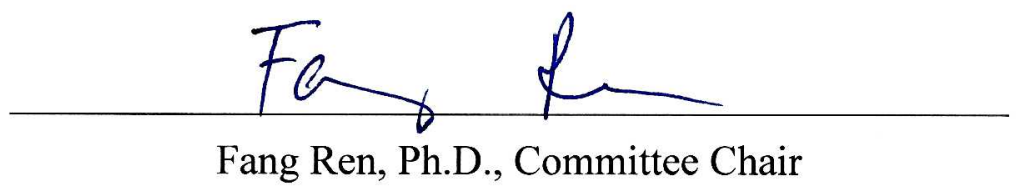

August 2010 



\section{Acknowledgements}

I would like to thank my second advisory committee member, Dr. Mark Kumler, for his addressing solar energy matters with their own spatial aspects. I would also like to thank Dr. Douglas Flewelling for his advice about conceptual approaches toward the solar energy matters. I am grateful to my client, Phillip Mielke, for his introducing an idea about taking advantage of LiDAR data in a solar energy mapping project. I am also grateful to Lynn Flewelling for her providing detailed edits of my report, and Stephen Daugherty for his technical advice. Of course, I would like to thank fellow students and staff in MS GIS Program for their encouragements and ideas. Lastly, I would like to give special thanks to my advisor, Dr. Fang Ren, for her insightful guidance and enthusiastic support of this project.

I would like to extend my deep appreciation to all people who have assisted me in spending this meaningful year in MS GIS Program. 



\begin{abstract}
Identifying and Viewing Rooftop Solar Potential:

A Case Study for the City of Redlands, California

by

Taira Nakanishi
\end{abstract}

Increasingly scarce resources pose new challenges to human development in the twentyfirst century. As a result of the shifting focus to renewable energy in order to meet the different needs of sustainable development, human society will inevitably experience numerous transformations on a variety of scales. Solar radiation plays a key role in achieving the objectives of sustainable development. Due to the potential variation over space and time, efficient utilization of solar energy requires that people understand the diverse spatial and temporal patterns of incoming solar radiation. This project was aimed at developing a Web-based solar map for the City of Redlands using DEMs generated from high resolution LiDAR data. This online solar map will provide the means to inform people of the latest updates regarding solar radiation, and also support their decisionmaking process in identifying ideal locations of solar panels for maximizing their benefits. 



\section{Table of Contents}

Chapter 1 - Introduction ............................................................................................... 1

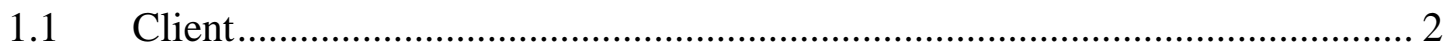

$1.2 \quad$ Problem Statement ............................................................................... 2

$1.3 \quad$ Proposed Solution .................................................................................. 2

1.3.1 Goals and Objectives ............................................................................ 2

1.3 .2 Scope

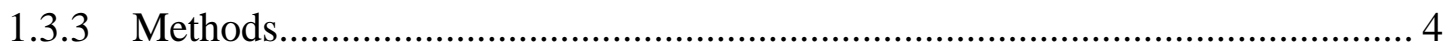

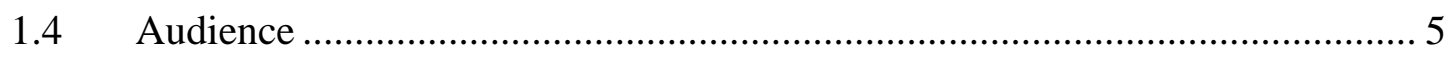

1.5 Overview of the Rest of this Report ............................................................ 5

Chapter 2 - Background and Literature Review .............................................................. 7

2.1 LiDAR Data for Solar Radiation Analysis ..................................................... 7

2.2 Web-based GIS Applications in Solar Energy Projects.................................... 8

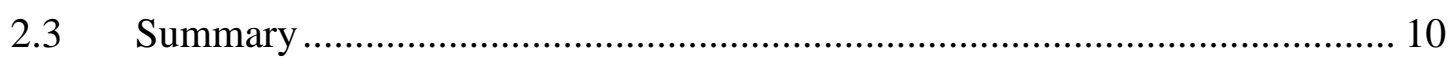

Chapter 3 - Systems Analysis and Design.................................................................... 11

$3.1 \quad$ Problem Statement .................................................................................... 11

$3.2 \quad$ Requirements Analysis ........................................................................ 11

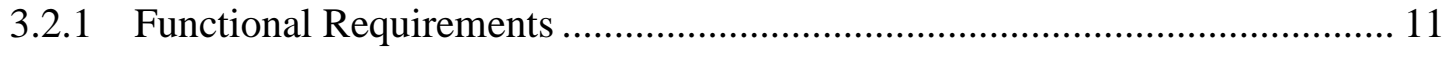

3.2.2 Non-functional Requirements .................................................................... 13

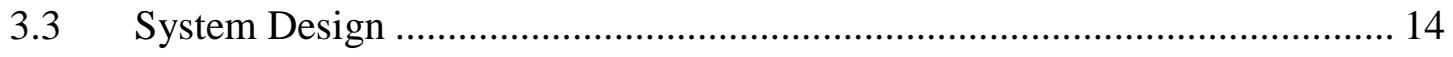

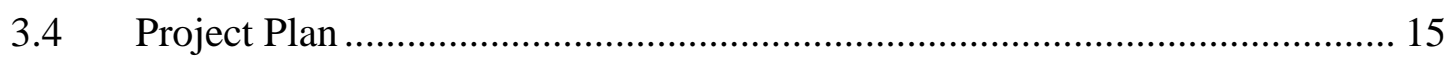

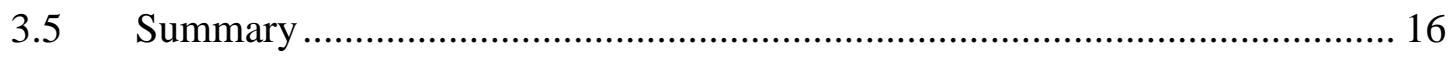


Chapter 4 - Database Design................................................................................................. 17

4.1 Conceptual Data Model ............................................................................. 17

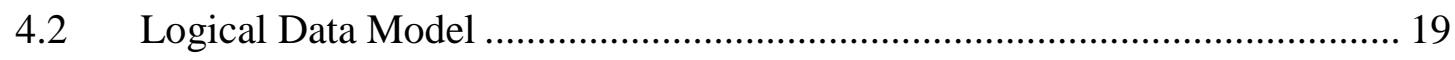

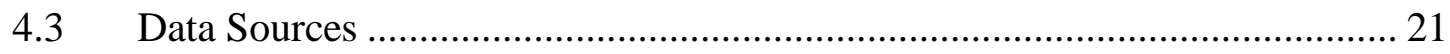

$4.4 \quad$ Data Scrubbing and Loading ....................................................................... 21

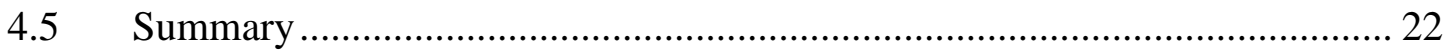

Chapter 5 - Implementation......................................................................................... 23

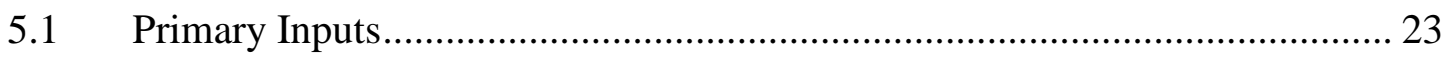

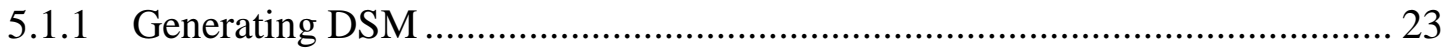

5.1.2 Generating Point Feature Class.................................................................... 25

$5.2 \quad$ Solar Radiation Analysis............................................................................... 27

5.2.1 Generating Point Feature Class................................................................... 28

5.2.2 Interpolating Solar Potential ....................................................................... 30

$5.3 \quad$ Web Mapping Application............................................................................ 31

5.3.1 Publishing a Map Service Using ArcCatalog ……………………………..... 31

5.3.2 Developing a Geoprocessing Service with ArcGIS Server Web ADF ............ 36

5.3.3 Developing a Web Application Using JavaScript API ..................................... 40

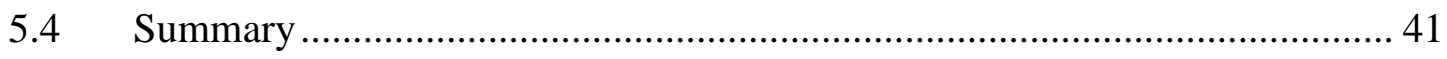

Chapter 6 - Results and Analysis................................................................................ 43

6.1 Primary Inputs Generated from LiDAR Point Clouds..................................... 43

6.2 Points Solar Radiation Analysis...................................................................... 45

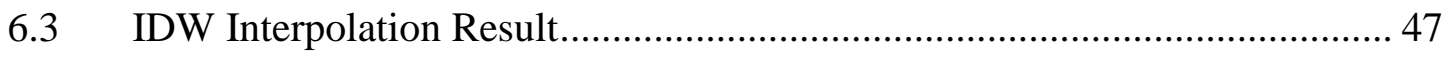

6.4 ArcGIS Server Web ADF Mapping Applications ........................................... 48 
6.5 ArcGIS API for JavaScript Mapping Applications ................................... 50

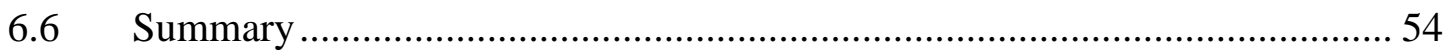

Chapter 7 - Conclusions and Future Work ............................................................. 55

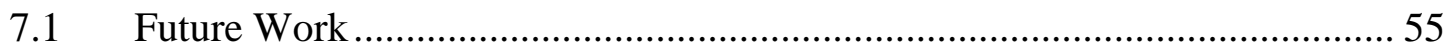

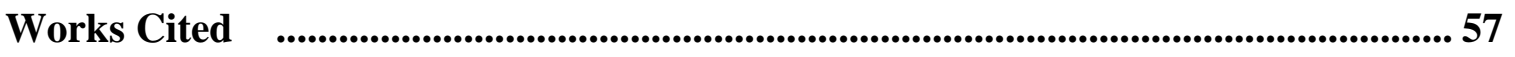

Appendix A. Web GIS Application Code ..................................................................... 61 



\section{Table of Figures}

Figure 1-1: Building Rooftops in the Study Area ............................................... 4

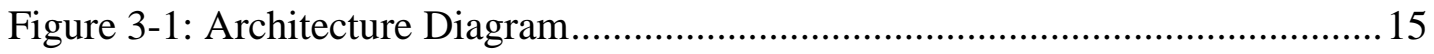

Figure 4-1: Solar Radiation Conceptual Data Model .......................................... 17

Figure 4-2: Classified Potential Obstructions ..................................................... 18

Figure 4-3: Solar Potential Installation Sites ..................................................... 19

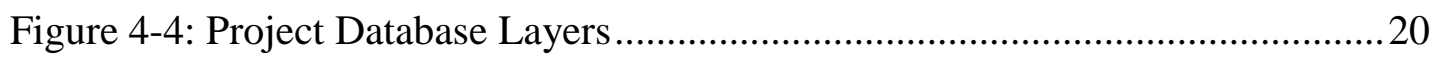

Figure 4-5: Point Spacing Information for Each LAS File...................................... 21

Figure 5-1: Overall Workflow for Generating Primary Inputs ...............................23

Figure 5-2: LAS to Multipoint Tool Interface for DSM.........................................24

Figure 5-3: Feature to Raster Tool Interface and Key Parameters ............................ 25

Figure 5-4: LAS to Multipoint Tool Interface for Sample Points ............................26

Figure 5-5: Exploding Multipart Feature (Top) to Singlepart Feature (Bottom).......27

Figure 5-6: Overall Workflow for Calculating Solar Radiation ...............................27

Figure 5-7: Points Solar Radiation Tool Interface and Key Parameters....................29

Figure 5-8: Calculate Field Tool Interface and Expression .................................... 30

Figure 5-9: IDW Tool with 3m Search Radius ................................................... 31

Figure 5-10: 2009GlobalSolarRadiation ArcMap Document Overview ....................32

Figure 5-11: 2009MonthlySolarlPotentials ArcMap Document Overview...............33

Figure 5-12: 2009GlobalSolarRadiation Map Service Information ......................... 34

Figure 5-13: 2009MonthlySolarPotentials Map Service Information ....................... 35

Figure 5-14: Selecting a Tiling Scheme for 2009GlobalSolarRadiation Map............ 36

Figure 5-15: Simplified Model to Publish as Geoprocessing Service ....................... 37 
Figure 5-16: Selecting a Synchronous Execution Type ...................................... 38

Figure 5-17: Selecting Map Services in ArcGIS Server Manager ........................... 39

Figure 5-18: Selecting a Geoprocessing Service in ArcGIS Server Manager .......... 40

Figure 6-1: 2m DSM (Top) and 30m DEM (Bottom) for the Study Area ................ 44

Figure 6-2: Sample Points on Building Rooftop Surfaces ................................... 45

Figure 6-3: Local Solar Radiation Pattern on South (Red, ID: 35517) and North

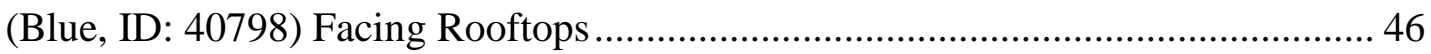

Figure 6-4: Seasonal Solar Radiation Pattern on South (Red) and North (Blue)

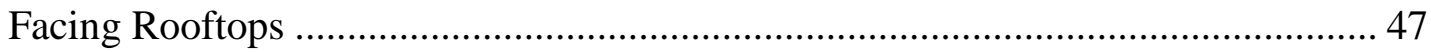

Figure 6-5: Continuous Surface Map of Solar Potential for January 2009 ............... 48

Figure 6-6: Display Monthly Solar Potential on Building Rooftops........................ 49

Figure 6-7: Calculate Solar Potential for Given Location On-the-Fly ..................... 50

Figure 6-8: University of Redlands Rooftop Solar Potential Viewer Interface ........ 51

Figure 6-9: Graduated Symbol for Monthly Solar Potential of December 2009 ...... 51

Figure 6-10: Solar Potential for Each Selected Samples in Table Tab .................... 52

Figure 6-11: Building Attribute Data in Buildings Tab ...................................... 53

Figure 6-12: Definition of Average Solar Potential Calculation in Definition Tab.. 53

Figure 6-13: Mean Solar Potential of Selected Samples in Chart Tab...................... 54 


\section{List of Tables}

Table 3-1. Functional Requirements for the Solar Radiation Analysis Model .......... 12

Table 3-2. Functional Requirements for the Web Application ................................. 12

Table 3-3. Non-functional Requirements for the Web-based Solar Map................... 13 



\section{List of Acronyms and Definitions}

$\begin{array}{ll}\text { ADF } & \text { Application Developer Framework } \\ \text { API } & \text { Application Programming Interface } \\ \text { ARRA } & \text { America Recovery and Reinvestment Act } \\ \text { ASCII } & \text { American Standard Code for Information Interchange } \\ \text { ASPRS } & \text { American Society for Photogrammetry and Remote Sensing } \\ \text { DEM } & \text { Digital Elevation Model } \\ \text { DOE } & \text { U.S. Department of Energy } \\ \text { DSM } & \text { Digital Surface Model } \\ \text { DTM } & \text { Digital Terrain Model } \\ \text { EECBG } & \text { Energy Efficiency and Conservation Block Grant } \\ \text { GIS } & \text { Geographic Information Systems } \\ \text { IDW } & \text { Inverse Distance Weighted } \\ \text { IT } & \text { Information Technology } \\ \text { LiDAR } & \text { Light Detection and Ranging } \\ \text { NAD } & \text { North American Datum } \\ \text { NAVD } & \text { North American Vertical Datum } \\ \text { REST } & \text { Representational State Transfer } \\ \text { SQL } & \text { Structured Query Language } \\ \text { UTM } & \text { Universal Transverse Mercator }\end{array}$





\section{Chapter 1 - Introduction}

Since the mid-twentieth century, the human population has been rapidly increasing. Although the total world population in 1950 was approximately 2.5 billion, the population quickly and steadily grew, reaching 6.5 billion by 2005 (United Nations, 2009). It is also estimated that the total world population will continue to increase and reach 9 billion by 2050 (United Nations, 2009). Due to the dramatic population increase, human activities have significantly altered many aspects of the Earth's environment (Bolstad, 2008a). Today, the term "sustainable development" is used in many settings of human society. Campagna (2006) states, "this concept [of sustainable development] implies that both technological and social settings should be organized so that human activities would not overload the capacity of the biosphere to absorb their impacts” (p. 3). Since people and the environment intersect with each other over space (Campagna, 2006), it is crucial for human society to address sustainable development problems with their own spatial aspect.

One important approach to achieving sustainable development is to take advantage of solar energy. The sun generates most of the energy required for all physical, biological, and industrial processes on the Earth (Dubayah \& Rich, 1996; Fu \& Rich, 1999; Podobnikar, Oštir, \& Zakšek, 2006; Ruiz-Arias, Pescador, Vázquez, \& Alsamamra, 2009). Because of the Earth's rotation and revolution about the sun, the amount of incoming solar radiation can vary depending on the latitude at various geographic locations (Fu \& Rich, 1999). At the same time topography such as elevation, surface orientation, and obstruction by neighboring surface features can also influence the distributions of incoming solar radiation (Fu \& Rich, 2002). Therefore, understanding this potential variation of incoming solar radiation across space can allow people to better use the sun as an energy source (Ruiz-Arias et al., 2009). Unlike oil, which has been vital for the economy for so long, solar radiation is so renewable that substituting solar energy for oil can most likely decrease the negative impacts on the environment of the Earth caused by the overuse of non-renewable energy sources (Podobnikar et al., 2006).

To date various new policies have encouraged local governments to make a number of significant changes in order to allow for the efficient utilization of solar energy. For instance, a grant program administered by the U.S. Department of Energy (DOE) enabled the City of Redlands to launch a new project for promoting photovoltaic installations. Through the Energy Efficiency and Conservation Block Grant (EECBG) Program, which was funded for the first time under the America Recovery and Reinvestment Act (ARRA) of 2009, the DOE awarded the city $\$ 678,000$. This grant allowed the Quality of Life Department to organize a project team that intended to promote photovoltaic installations and improve the energy efficiency. This project focused on developing new tools to analyze solar energy variation at different geographic locations and communicate the results with policy makers in a user-friendly manner. 


\subsection{Client}

The client for this project was Mr. Philip Mielke of the City of Redlands, California. In 2007, the City of Redlands established the Information Technology (IT) department which consists of the MIS division, which mainly deals with network and application issues, and the Geographic Information Systems (GIS) division, which manages GISrelated matters. Mr. Mielke is the GIS Administrator of the GIS division. He has been involved in various projects such as crime analysis and economic development research in the city, and numerous tasks including GIS data archiving and map production. The GIS division makes spatial data and information available to citizens and business personnel in the city.

\subsection{Problem Statement}

In order to assist in the decision-making process of the City of Redlands on the installation of solar panels, the client recognized a need to develop an interactive tool that can analyze and display the latest updates of relevant information about solar energy. More importantly, due to unique surface features of each rooftop, it is critical to reveal variations in the amount of solar radiation and identify appropriate parts of buildings' rooftops for the installation of solar panels.

\subsection{Proposed Solution}

Surface analysis for determining the amount of incoming solar radiation is complex. Not only do a variety of geographic factors including elevation and orientation, such as slope and aspect, influence the solar energy input, but also the changing sun's azimuth and altitude. By using high resolution LiDAR data as primary inputs, however, GIS can assist in conducting complex surface analysis and providing crucial spatial information on the solar radiation. At the same time, a Web-based GIS application can be very useful in order to increase accessibility for a wide range of audiences. In general, the proposed solution to the project was:

- To create a model that produces raster datasets of varying incoming solar radiation across surfaces of each building rooftop.

- To develop a Web-based GIS application using ArcGIS API for JavaScript (JavaScript API) and distribute the analysis results to various groups of people.

\subsubsection{Goals and Objectives}

The main goal of this project was to develop a Web-based solar map that displays local and seasonal patterns of solar potential that were calculated using digital elevation models (DEMs) as primary inputs. The roles of this online solar map are twofold: to identify suitable locations for mounting solar panels on building rooftops, and to inform users of the latest updates of incoming solar radiation on each rooftop on a monthly basis. 


\subsubsection{Scope}

This project intended to produce two major deliverables: a model that runs a sequence of algorithms to calculate the incoming solar radiation on building rooftops, and a Web application that displays the analysis results in a user-friendly manner. This study originally intended to use high resolution LiDAR data that were captured in the fall of 2009 and building footprint data for the entire City of Redlands. However, because the LiDAR data were not provided on time, the scope of the project was scaled down to using the LiDAR data for the University of Redlands campus.

The University of Redlands is located in the northeastern part of the City of Redlands, California and is approximately 160 -acres with a gentle slope ranging from 439 to $451 \mathrm{~m}$ above sea level. Of all the facilities in this study area, 114 building rooftops were examined in order to reveal varying incoming solar radiation on a monthly basis (Figure 1-1). Because the city owns the same types of primary inputs, including LiDAR data and building footprint data, the model and Web application for this study area can be applicable to the entire city. 


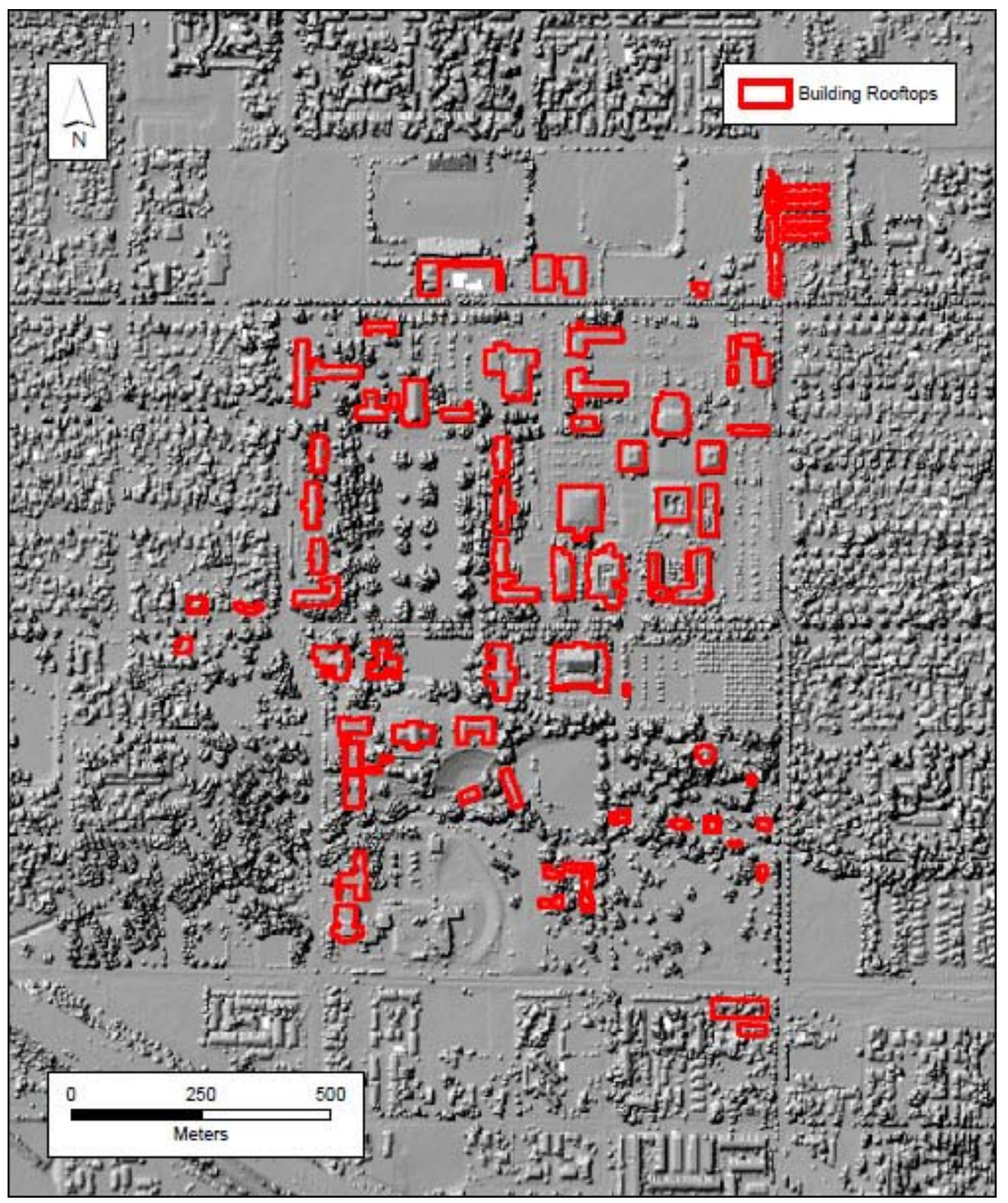

Figure 1-1: Building Rooftops in the Study Area

\subsubsection{Methods}

This project comprised two major tasks: developing the Solar Radiation Analysis model and a Web-based GIS application. Solar radiation analysis was performed by a sequence of algorithms created using the ArcGIS ModelBuilder. The most critical part of the model was the Points Solar Radiation tool in the Spatial Analyst toolbox. Prior to running the Points Solar Radiation tool, this model first converted LiDAR data to a digital surface model (DSM) and a multipoint feature class masked by outlines of the buildings, which was further converted to a singlepart point feature class. After running the Points Solar Radiation tool using these primary inputs, this model further interpolated the output point 
features to raster datasets to reveal varying incoming solar radiation across the entire surfaces of building rooftops.

The Web-based solar map employed JavaScript API as the development platform. To begin, the raster datasets that were created by the Solar Radiation Analysis model were added to ArcMap documents. The maps were saved as mxd files and published to ArcGIS Server 9.3. This online solar map consists of three main Representational State Transfer (REST) functionalities. Fielding (2000) defines REST as "an abstraction of the architectural elements within a distributed hypermedia system.” The three proposed REST functionalities included: identify map service in order to select a target building's rooftop, execute a task in order to calculate the amount of incoming solar radiation on a selected building rooftop, and illustrate a map service for the temporal variation of solar radiation on the rooftop in chart and table form.

\subsection{Audience}

This report is first of all intended for the City of Redlands' GIS Division in the IT Department, the Quality of Life Department, the City Council members, and a group of people who work on solar energy matters in the city. The University of Redlands, including the Redlands Institute and the office of Marketing and Strategic Communications, can be another potential audience of this report. This report uses common GIS terminology in order to describe technical requirements and functionalities. However, this report does not necessarily require audiences to have a high-level understanding of GIS technology.

\subsection{Overview of the Rest of this Report}

The first chapter of this report illustrated the overview of this project, "Identifying and Viewing Rooftop Solar Potential - A Case Study for the City of Redlands, California.” Chapter 2 reviews the past literature on LiDAR data for solar radiation analysis and Webbased GIS applications and further presents an argument for meeting the specific needs of the solar radiation analysis by using two different mapping examples. Chapter 3 demonstrates the initially proposed project plan and discusses necessary modifications made as the project progressed. Chapter 4 addresses the database design using the conceptual and logical data models and further describes data sources. Chapter 5 describes the required procedures in order to implement the previously proposed solutions for the problem. Chapter 6 discusses the implications of findings and results for solar radiation analysis and Web-based GIS applications. Lastly, Chapter 7 draws a conclusion by recapping the project's success and providing recommendations and potential avenues for future studies. 



\section{Chapter 2 - Background and Literature Review}

As a result of the shifting focus to renewable energy in order to meet the different demands of sustainable development, people have become more aware of the need for measuring the potential amount of incoming solar radiation at specific locations. In this regard, solar maps can provide users with a better understanding of various benefits from utilizing the solar energy (Kandt et al., 2009). Web-based solar maps have become more popular because of their advantages over traditional paper maps. This chapter presents a literature review that addresses two key elements regarding the development of a Webbased solar map for the City of Redlands. The literature examined here involves the use of LiDAR data for solar radiation analysis, and Web-based GIS applications.

\subsection{LiDAR Data for Solar Radiation Analysis}

Surface analyses of solar radiation can be performed based on DEMs. Chang (2005) defines DEM as "a digital model with an array of uniformly spaced elevation data in raster format” (p. 90). ArcGIS Solar Radiation tools were developed in order to assist in calculating the amount of solar radiation (Fu \& Rich, 1999). Using DEMs as primary inputs, these solar radiation modeling tools yield the spatial and temporal patterns of solar radiation, considering the influences of the viewshed, surface orientation, elevation, and atmospheric conditions (Fu \& Rich, 1999). The solar radiation modeling tools can be categorized into two types: Area Solar Radiation and Points Solar Radiation. These can calculate the incoming solar radiation for a geographic area or a specific location respectively (Fu \& Rich, 2002; ESRI, 2010a). DEMs can also be used in the Solar Radiation Graphics tool. Unlike the Area or Points Solar Radiation tools, outputs from this tool are "representations of directions in a hemisphere of directions looking upward from a given location" (ESRI, 2010b). This tool can assist in better understanding significant factors of viewshed and information on atmospheric conditions (ESRI, 2010b).

As the primary input, the quality of DEMs greatly influences the models' accuracy of solar radiation analysis. Not only does it directly determine the terrain elevation, but it also affects other terrain characteristics, including slope and aspect. One study showed that, compared to elevation and viewshed, the inaccurate surface orientation due to poor quality of DEMs can potentially cause more significant errors in solar radiation analysis because the amount of incoming solar radiation is measured based on angle of incidence, which is determined by surface slope and aspect (Fu \& Rich, 1999). In general, 30m USGS DEMs can be considered as sufficient for most environmental and ecological applications (Fu \& Rich, 1999). However, this relatively coarse resolution makes it hard to detect the detailed characteristics of surface features (Elaksher \& Bethel, 2002). Therefore, it is recommended that DEMs of higher resolution be used to detect more detailed surface features and improve the model's accuracy of solar radiation analysis (Fu \& Rich, 1999).

LiDAR systems have become increasingly popular due to their own advantage over a traditional photogrammetry technique using a "stereopair" (Bolstad, 2008b). The LiDAR 
system is an active laser system that releases laser pulses directed at the Earth's surface and detects the returned energy (Bolstad, 2008b; Lillesand et al., 2008). The surface elevations are measured based on the time required for laser pulses to reach a target and return (Lillesand et al., 2008; Lloyd \& Atkinson, 2006). Since its initial development in the 1970s, LiDAR systems have been increasingly preferable for collecting elevation data because of their higher productivity, resolution, and accuracy (Taylor et al., 2007). Further, the recent advanced sensing technology enables LiDAR systems to increase point density of datasets (Rottensteiner, 2003). With a high density of point clouds, LiDAR data are applied to a wide range of fields, such as generating high-quality DEMs (Ma, 2005) and creating 3D modeling of landscapes in urban settings (Rottensteiner, 2003).

LiDAR systems can detect multiple returning pulses from the ground and above ground surface features. LiDAR point data include not only elevation data at the ground surface, but also those for the features above the ground such as trees and buildings. In order to generate bare-earth DEMs that indicate the ground surface elevations, only the last pulse from the ground surface needs to be extracted through data filtering (Lloyd \& Atkinson, 2006; Ma, 2005). In the same manner, the multiple returning pulses, especially the early returns, from each LiDAR point data can be also extracted in order to reveal the various surface features, including trees and shrubs (Lillesand et al., 2008). These extracted laser pulses received from each LiDAR point data then can be converted to DEMs (Lloyd \& Atkinson, 2006). Therefore, the quality of DEMs generated with LiDAR data largely depends on the accuracy of LiDAR point data segmentation (Lloyd \& Atkinson, 2006; Ma, 2005). In general, the enhanced DEMs are critical input in conducting surface analyses of solar radiation because of the higher point density and ability to detect multiple returning pulses of LiDAR systems.

LiDAR data are commonly delivered as a collection of files in either ASCII (American Standard Code for Information Interchange) or LAS file format (ASPRS, 2010; ESRI, 2010c). The LAS file format, which is an industry standard binary file format for the interchange of LiDAR data, is regarded as better alternative to a generic ASCII file interchange system. LAS files can retain information specific to LiDAR data in a simplistic manner (ASPRS, 2010). In comparison with ASCII files, LAS files are not only faster at reading and interpreting elevation data, but are also capable of storing a larger amount of data, including meaningful headers that contain information about the data in a smaller file (ESRI, 2010c). Because of the higher performance level and larger storage capacity, LAS files are often considered as a preferable LiDAR file format today (ESRI, 2010c).

\subsection{Web-based GIS Applications in Solar Energy Projects}

Web-based GIS enhances the ability to present spatial information analysis. Due to the rapid progress of information technology, various types of GIS are available today, ranging from the traditional GIS, such as mainframe GIS and desktop GIS; distributed GIS, including Internet GIS, more particularly Web-based GIS; and mobile GIS (Peng \& Tsou, 2003). Unlike traditional GIS, a Web-based GIS allows users to explore spatial information on the Internet and wireless network without installing GIS programs in the 
same mainframe or desktop computer (Peng \& Tsou, 2003). Different types of GIS are still used interchangeably depending on the circumstances. However, Web-based GIS, which use the World Wide Web (WWW) as the primary means of performing GIS analysis (Dragićević, 2004; Peng \& Tsou, 2003), have increasingly become an integral part of today’s GIS industry.

Web-based GIS have been implemented in various solar energy projects around the world. In 2007, the City of Boston, Massachusetts, launched a two-year \$550,000 project, “Solar Boston," under the Solar America Initiative of the DOE. This project aimed to minimize negative impacts on the environment by promoting using buildings' renewable energy sources. The city also intended to increase the amount of solar energy utilized to 25 megawatts by 2015. In order to achieve these goals, the city recognized a need to develop a tool for communicating information about the solar energy and, as a result, involved the development of the Solar Boston map as a significant feature of this program (City of Boston, 2009).

The Solar Boston Map employed ArcGIS solar radiation tools exclusively to calculate the potential solar radiation of each building rooftop (DeMeritt, 2008; Kandt et al., 2009). The digital terrain models (DTMs) of the city were first combined with the building height data by using the first return of LiDAR values. These three-dimensional DSMs of the city's landscape were used as the primary inputs of the solar radiation analysis. The Points Solar Radiation tool in ArcGIS Spatial Analyst toolbox was then applied to determine the amount of potential solar radiation on each rooftop, given the assumption that all roofs are flat (Kandt et al., 2009). Finally, Boston’s Web GIS application was developed using ArcGIS API for Flex. This technology enabled the city to display not only information about locations of ongoing renewable energy projects, but also estimated the monthly solar potential and annual cost and carbon dioxide savings of each building rooftop (City of Boston, 2009; DeMeritt, 2008).

Similarly, Los Angeles County, California, developed a Web-based solar map, known as the LA County Solar Map. This map, which covers approximately 3,000 square miles of geographic area, was, as of the year of 2009, regarded as the largest solar map in the world (Los Angeles County, 2009). This solar map can measure the characteristics of roof slope, pitch, distinct rooftop structures such as chimneys and walls, as well as trees and buildings in surrounding areas. Based on the measurement results, this map can be also used to calculate the amount of potential solar radiation on each building rooftop (Los Angeles County, 2009). As a result, users can enter an address into the map website and easily access a variety of information on the solar radiation analysis. This map also provides the total area of their roofs, the area of usable rooftop for solar power generation, and the amount of annual savings of electricity and carbon. The county contends that this solar map will assist in reducing greenhouse gas emissions and energy costs in the future (Los Angeles County, 2009). 


\subsection{Summary}

Web-based maps using GIS technology can assist in conducting solar radiation analysis and provide significant information to various users. DEMs, which are generated from LiDAR data, can be used as primary inputs of solar radiation analysis in order to reveal spatial and temporal patterns of incoming solar radiation at the Earth's surface. A higher resolution DEM makes it possible to detect more detailed surface features at local and landscape scales. At the same time, Web-based GIS applications can play a key role as an efficient means to distribute the information to various users. In the next chapter, the systems analysis and design that were required for the Redlands' Web-based solar map will be further examined based on lessons learned from the literature review. 


\section{Chapter 3 - Systems Analysis and Design}

Fully understanding the client's needs is the first and a vital task for successful GIS planning. A good project plan usually starts with a system design that will meet the client's expectations and minimize the negative impacts of various potential constraints. This chapter examines the system design for the Web-based solar mapping project by recapping the problem statement of the project, discussing both functional and nonfunctional requirements for the project completion, and describing the system architecture. This chapter also discusses the initial project plan and necessary modifications to the project plan that occurred as the project evolved.

\subsection{Problem Statement}

In order to assist in the decision-making process of the City of Redlands regarding the installation of solar panels, the client recognized the need to develop an interactive tool that can analyze and display the latest updates of relevant information about solar energy. More importantly, due to the unique surface features of each building rooftop, it was critical to understand local and seasonal patterns of solar radiation across the building rooftops and to identify appropriate parts of the rooftops for the installation of solar panels.

\subsection{Requirements Analysis}

This project focused on two major deliverables: a model that runs a sequence of algorithms and calculates the incoming solar radiation on building rooftops, and a Web application that displays the analysis results in a user-friendly manner. To successfully deliver these products to the client, a requirement analysis was first conducted. Outcomes of the requirement analysis were crucial for system design to meet the client's expectations as well as resolve technical limitations. Accordingly, this section addresses both functional and non-functional requirements for this project.

\subsubsection{Functional Requirements}

Table 3-1 summarizes the functional requirements for the Solar Radiation Analysis model. These requirements include the functionalities of the model that were identified considering potential use cases. First, the client and other staff in the GIS Division need to be able to select and convert LiDAR point clouds to a DSM and a sample locations point feature class. Using the DSM and sample locations point feature class, as well as the building footprint data, the Solar Radiation Analysis model should create twelve raster datasets to show local variations of the solar radiation by month. At the same time, the parameters of the Points Solar Radiation tool should be adjustable for future modifications. The adjustable parameters include: latitude, sky size/resolution, time configuration, and calculation directions. Therefore, if this model is used under different circumstances in the future, the users can modify these parameters accordingly. 
Table 3-1. Functional Requirements for the Solar Radiation Analysis Model

\begin{tabular}{|c|c|}
\hline Requirement & Description \\
\hline $\begin{array}{l}\text { Select and input LiDAR data and building } \\
\text { footprint data }\end{array}$ & $\begin{array}{l}\text { To enable users to select LAS files and } \\
\text { polygon feature class to process }\end{array}$ \\
\hline $\begin{array}{l}\text { Convert the LAS files to multipoint feature } \\
\text { class }\end{array}$ & $\begin{array}{l}\text { To enable users to change the format of } \\
\text { LiDAR data for using in ArcGIS } \\
\text { environment while select appropriate class } \\
\text { code(s) and projection }\end{array}$ \\
\hline $\begin{array}{l}\text { Convert multipoint features to raster } \\
\text { dataset }\end{array}$ & $\begin{array}{l}\text { To enable users to change the format of } \\
\text { data for the Points Solar Radiation tool }\end{array}$ \\
\hline $\begin{array}{l}\text { Intersect multipoint features by building } \\
\text { footprint data }\end{array}$ & $\begin{array}{l}\text { To enable users to spatially extract LiDAR } \\
\text { point data in the building class code }\end{array}$ \\
\hline $\begin{array}{l}\text { Convert multipoint features to single point } \\
\text { features }\end{array}$ & $\begin{array}{l}\text { To enable users to change the format of } \\
\text { data for the Points Solar Radiation } \\
\text { Analysis tool }\end{array}$ \\
\hline $\begin{array}{l}\text { Calculate the amount of incoming solar } \\
\text { radiation }\end{array}$ & $\begin{array}{l}\text { To enable users to calculate the amount of } \\
\text { incoming solar radiation and add new } \\
\text { attribute fields to the input point feature } \\
\text { class }\end{array}$ \\
\hline $\begin{array}{l}\text { Interpolate the amount of incoming solar } \\
\text { radiation }\end{array}$ & $\begin{array}{l}\text { To enable users to estimate the amount of } \\
\text { incoming solar radiation across rooftop } \\
\text { surfaces based on the sampled values }\end{array}$ \\
\hline
\end{tabular}

Similarly, Table 3-2 lists the functional requirements for the Web application using ArcGIS API for JavaScript (JavaScript API). These requirements address the functionalities of the Web application that were identified considering potential use cases. The Web application should allow users to toggle on and off map layers of interest using the radio buttons. Users can also identify a target rooftop in order to calculate the amount of incoming solar radiation and display the analysis result in both chart and table form. Due to the high density of point data within each rooftop outline polygon feature, it was preferable for users to sufficiently zoom in on a target rooftop when identifying individual point data on the map.

Table 3-2. Functional Requirements for the Web Application

\begin{tabular}{|l|l|}
\hline Requirement & Description \\
\hline Select and display a map on the Web & $\begin{array}{l}\text { To enable users to select and display a map } \\
\text { of interest on the Web }\end{array}$ \\
\hline $\begin{array}{l}\text { Select a target feature and execute the solar } \\
\text { potential calculation }\end{array}$ & $\begin{array}{l}\text { To enable users to identify a rooftop of } \\
\text { interest by pointing the feature and view } \\
\text { attributes about monthly solar potentials, } \\
\text { building name and area of rooftop }\end{array}$ \\
\hline $\begin{array}{l}\text { Display the attributes in chart and table } \\
\text { form }\end{array}$ & $\begin{array}{l}\text { To enable users to view the attributes in a } \\
\text { chart and table form }\end{array}$ \\
\hline
\end{tabular}




\subsubsection{Non-functional Requirements}

Table 3-3 summarizes the non-functional requirements for the Solar Radiation Analysis model and the Web application. Non-functional requirements involved technical, operational, and transitional requirements that were determined based on potential use cases. First, the technical requirements included the minimum software specifications necessary to use the Solar Radiation Analysis model and the Web application. In order to run the Solar Radiation Analysis model, the client's computer needed ArcGIS Desktop 9.3 or higher. The client's computer also must have the 3D Analyst and Spatial Analyst extensions because the model includes the LAS to Multipoint and Points Solar Radiation tools that are available in the 3D Analyst and Spatial Analyst toolboxes respectively. At the same time, in order to author and publish map services, the Web application required the ArcGIS Server 9.3 with the Spatial Analyst extension. This computing environment would enable the client not only to display the analysis result but also to calculate the solar potential of each rooftop.

Table 3-3. Non-functional Requirements for the Web-based Solar Map

\begin{tabular}{|c|c|}
\hline Requirement & Description \\
\hline \multicolumn{2}{|l|}{ Technical } \\
\hline $\begin{array}{l}\text { ArcGIS Desktop } 9.3 \text { with 3D Analyst and } \\
\text { Spatial Analyst extensions }\end{array}$ & $\begin{array}{l}\text { The Solar Radiation Analysis Model works } \\
\text { with ArcGIS Desktop } 9.3 \text { with 3D Analyst } \\
\text { and Spatial Analyst extensions, as the tools } \\
\text { required this environment }\end{array}$ \\
\hline $\begin{array}{l}\text { ArcGIS Server } 9.3 \text { with Spatial Analyst } \\
\text { extension }\end{array}$ & $\begin{array}{l}\text { The Web applications work with ArcGIS } \\
\text { Server } 9.3 \text { with Spatial Analyst extension, } \\
\text { as the tools required this environment }\end{array}$ \\
\hline \multicolumn{2}{|l|}{ Operational } \\
\hline $\begin{array}{l}\text { Speedy and efficient data processing and } \\
\text { analysis }\end{array}$ & $\begin{array}{l}\text { The solar potential calculation using } 1 \mathrm{~m} \\
\text { resolution LiDAR data needs to be } \\
\text { completed with detailed surface features } \\
\text { while keeping the required amount of time } \\
\text { reasonable }\end{array}$ \\
\hline $\begin{array}{l}\text { Model's applicability to different primary } \\
\text { inputs }\end{array}$ & $\begin{array}{l}\text { The model needs to be applicable to the } \\
\text { entire city by using different data sets as } \\
\text { primary inputs }\end{array}$ \\
\hline \multicolumn{2}{|l|}{ Transitional } \\
\hline Demonstration & $\begin{array}{l}\text { Brief demonstration on how to operate and } \\
\text { maintain the model and Web applications }\end{array}$ \\
\hline Documentations & $\begin{array}{l}\text { Detailed MIP report and digital copies of } \\
\text { relevant information that can be accessible }\end{array}$ \\
\hline
\end{tabular}

Second, the operational requirements pertained to the efficiency of the system. In comparison with 30m USGS DEMs, for example, the use of high resolution LiDAR point clouds requires more time to generate a DSM and a sample locations point feature class. Therefore, it was critical to determine an appropriate cell size that would enable the 
model to reveal detailed rooftop surface features while keeping the processing time reasonable. At the same time, despite the fact that computing capacity has been improved enough to accept higher values for necessary parameters, it was still important to consider keeping these values reasonable and run the model within a reasonable amount of time. Further, the model had to run correctly regardless of primary inputs. This confirms that the model for this study area can be applicable to the entire city.

Lastly, the transitional requirements included creating documentation of the final products and providing the client with a brief demonstration. This project needed to illustrate the procedure of the Solar Radiation Analysis model and Web-based solar map. These documentations were distributed as a part of the MIP report. In addition to the documentation, a brief demonstration was required to ensure that the client is able to use and maintain the final products. Demonstration was a major element for project completion because the client would need to demonstrate to other potential users how to operate the Web-based solar map and also maintain the Solar Radiation Analysis model for future updates.

\subsection{System Design}

The system design for this project was determined based on the outcome of the requirements analysis. The Web-based solar map consists of three major components/layers: user layer, business layer, and data layer (Figure 3-1). Potential users, including citizens, city staff in the GIS Division and the Quality of Life Department, City Council members, and people working for solar energy solutions in the city, can view the solar potential maps via the city's Web site. The city staff can directly access the city's geodatabase that stores the model and primary inputs, such as LiDAR data and building footprint data. They maintain the data and update the solar radiation calculation using the Solar Radiation Analysis model in ArcGIS Desktop 9.3 or higher. At the same time, the ArcGIS Server 9.3 or higher, in conjunction with the Spatial Analyst extension, was required in order for the GIS Division staff to access the published map services. 


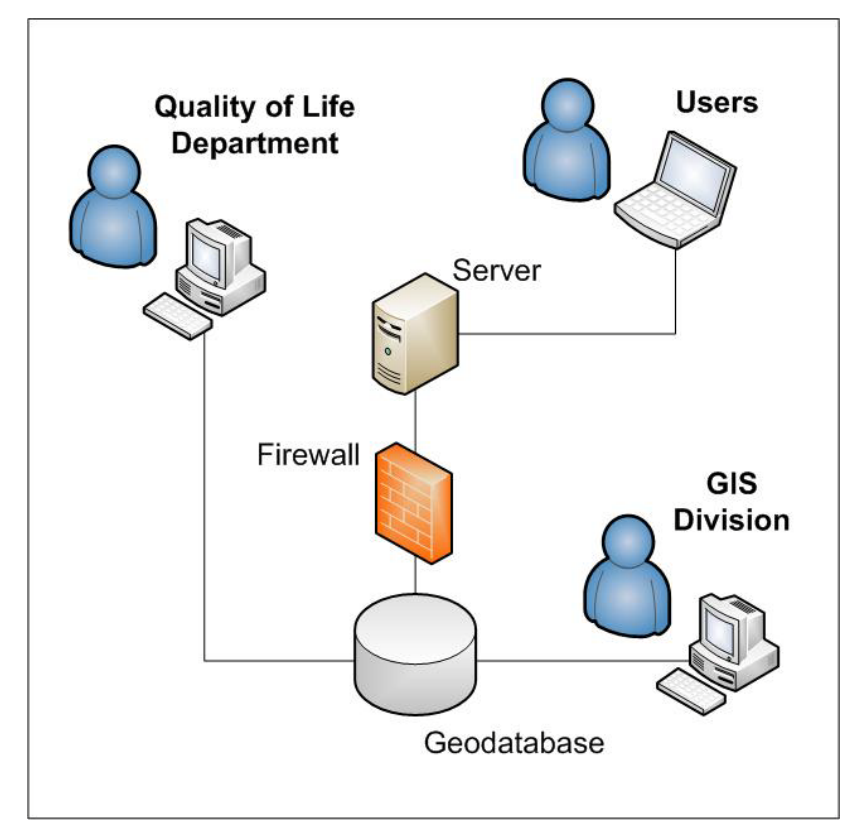

Figure 3-1: Architecture Diagram

\subsection{Project Plan}

To achieve the project's goals in a timely manner, the project plan initially consisted of five major phases, with an estimated time period needed to complete each task. The initial proposed project plan included:

- Phase 1: Requirements Analysis and Data Acquisition Preliminary research was conducted in order to understand the functional and non-functional requirements. The research involved reviewing literature relevant to the solar radiation analysis and examining currently available Web-based solar maps.

- Phase 2: Geoprocessing Model Development

The Solar Radiation Analysis model was developed by using the ArcGIS ModelBuilder. Necessary tools and parameters were determined based on the outcomes from the preliminary research. Interviews with the client also helped to better understand the requirements for the analysis model.

- Phase 3: Web Application Development

The Web application was developed using the JavaScript API. The key functions included identifying a target rooftop and calculating the amount of solar potential on the selected building rooftop.

- Phase 4: Testing and Monitoring Necessary modifications regarding the Solar Radiation Analysis model and Web application were discussed in order to meet the client's expectations. 
- $\quad$ Phase 5: ESRI User Conference

The project results were presented at the paper and poster sessions in the ESRI User Conference.

Necessary modifications occurred to the plan as the project progressed. This study originally intended to use high resolution LiDAR data that were captured in the fall of 2009 and existing building footprint data for the entire City of Redlands. However, because the LiDAR data were not provided on time, the scope of the project was scaled down to using only the LiDAR data for the University of Redlands campus. At the same time, the LiDAR data processing task was removed from the project plan because the LiDAR point clouds for the university campus had been appropriately separated by a private vendor based on different class codes of surface features.

The original project plan aimed to develop a Web application that can display a variety of information, such as locations of installation sites, the estimated daily solar potential, and the annual cost and carbon dioxide savings of each building rooftop. Due to the time constraints, however, this project involved only three significant functions, including identifying a building rooftop, calculating the amount of monthly solar radiation on a selected building rooftop, and displaying the results in both chart and table form. More significantly, the development framework was also changed from the API for Flex to JavaScript API. This technical shift considerably increased the amount of time to complete the Web application development task.

\subsection{Summary}

This chapter discussed the systems analysis and design, as well as the project plans, with necessary modifications. The requirements analysis was crucial for addressing the goals and objectives that the project was intended to achieve. The analysis was conducted focusing on both functional and non-functional requirements, including technical, operational, and transitional requirements. The system analysis result indicated that this project involved various requirements in order to conduct the solar radiation analysis and display the analysis result on the Web. An original project plan illustrated required tasks with their proposed milestones. Necessary changes were added to the initial project plan in order to complete the entire project in a timely manner. 


\section{Chapter 4 - Database Design}

This chapter discusses four key components of database design for the Web-based solar mapping project. First, three conceptual data models are discussed. These conceptual data models are aimed at defining significant entities of this project and representing associations between the entities. Second, a logical data model is examined. The logical data model was required in order to implement the entities and associations represented by the conceptual data models. Third, data sources of this project are described. Two primary inputs to this project were the LiDAR data that contain elevation of the University of Redlands campus and a campus building polygon feature class. Lastly, procedures for data scrubbing and loading are discussed.

\subsection{Conceptual Data Model}

The sun radiates energy continuously, but incoming solar radiation across the space varies. To model the how the incoming solar radiation varies on the rooftops, three conceptual models were developed in the project. A conceptual data model is a high-level abstraction that illustrates the complex real world in a simplistic way. The first conceptual data model (Figure 4-1) describes the important factors that affect spatial and temporal patterns of incoming solar radiation at the Earth's surface. The foremost entities include the sun, installation, obstruction, and terrain.

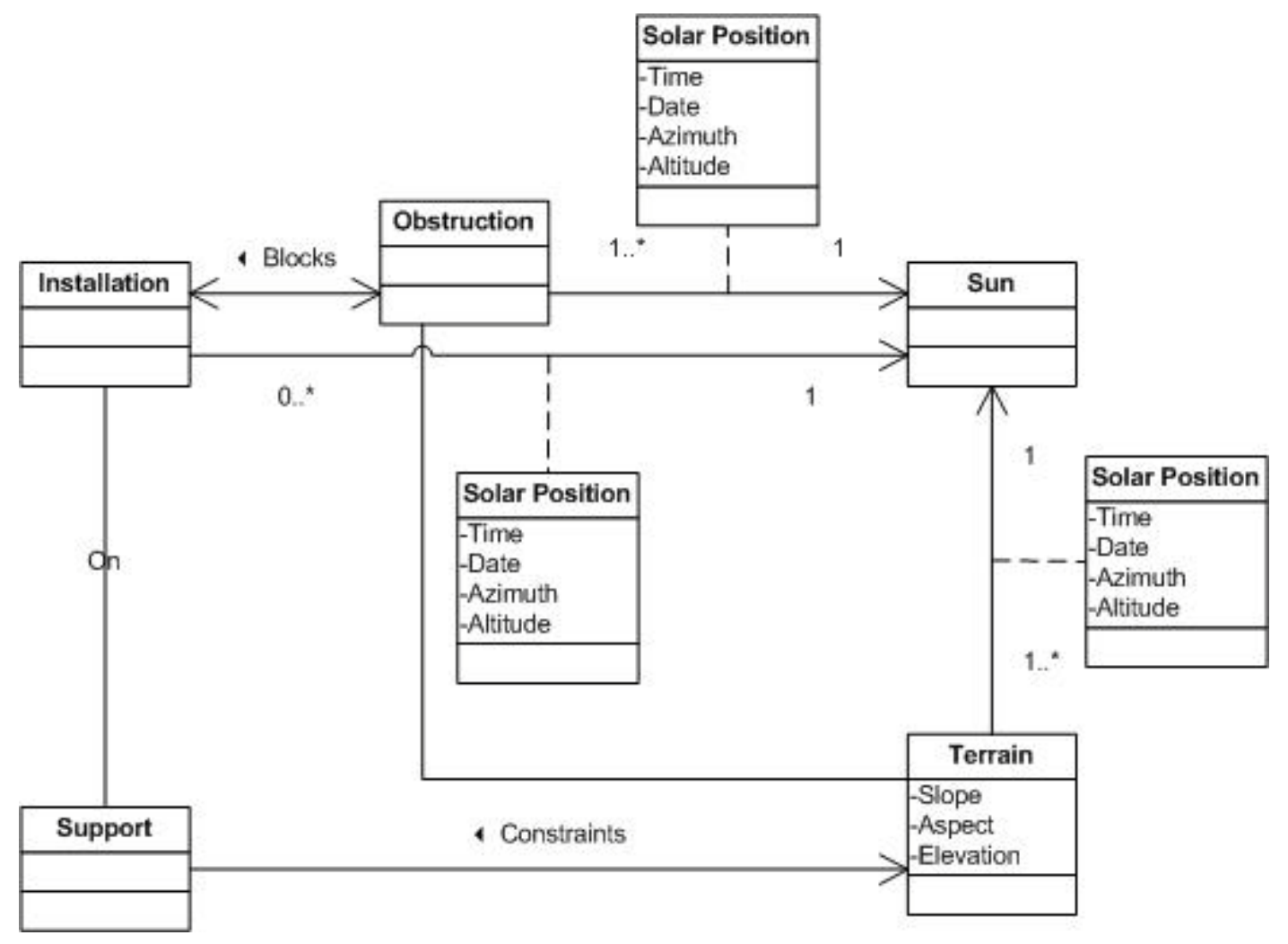

Figure 4-1: Solar Radiation Conceptual Data Model 
The second conceptual data model (Figure 4-2) illustrates various features that can potentially obstruct the incoming solar radiation at the Earth's surface. Three major obstacles, including buildings, trees, and terrain features, can modify the amount of incoming solar radiation at installation sites. The canopy class was also included in this conceptual data model because canopies can significantly affect the incoming solar radiation under some circumstances. Hillshade and cast shade are two types of shadows (Podobnikar et al., 2006). The hillshade occurs "when the surface is oriented away from the [sun]" (Podobnikar et al., 2006, p. 420). The cast shade occurs "when an obstacle between the sun and the [Earth's] surface is present” (Podobnikar et al., 2006, p. 420). In other words, even if the surface is oriented toward the sun, surface features such as vegetation, terrain, and human structures can make the surface partially/entirely insulated. Therefore, varying incoming solar radiation at installation sites depend largely on height/elevation, size, and location of obstacles.

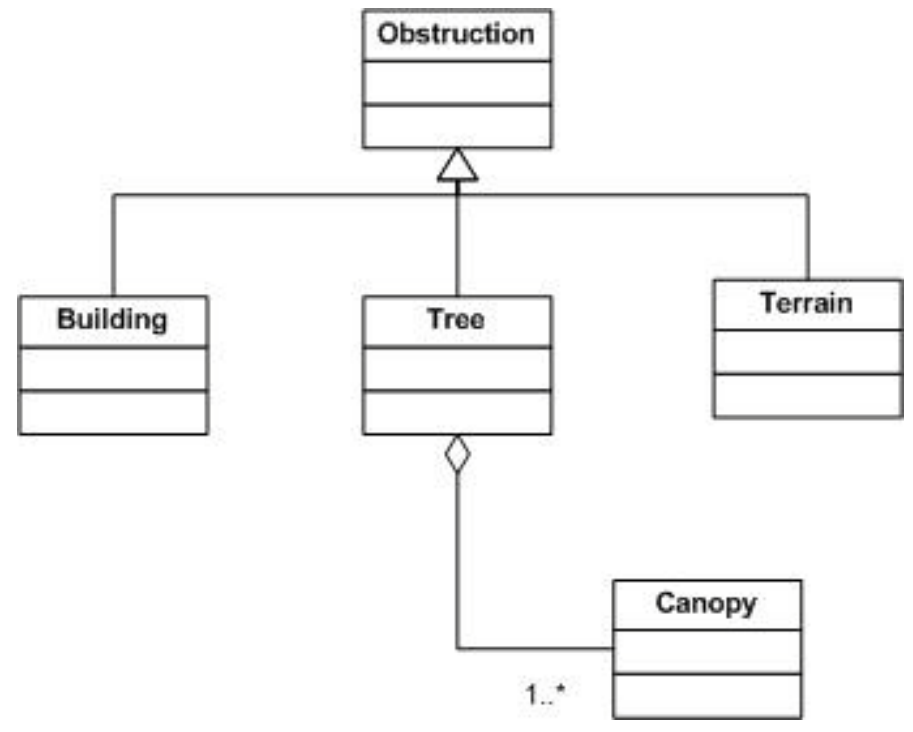

Figure 4-2: Classified Potential Obstructions

The third conceptual data model (Figure 4-3) describes installation sites for solar panels. Of all the potential differences, geographic location for mounting solar panels is considered as the most significant factor to identify appropriate installation sites. Due to the potential influences of different types of obstructions, solar panels are commonly installed on supports. They can be buildings, rooftops, or foundations. The terrain features constrain the location of supports. It is also possible that one support can be supported by another. Their height and orientation are defined in accordance with circumstances at each site. With the increased solar potential afforded by supports, the suitability of installation sites can ultimately be determined by elevation, surface orientation, and shadows cast by neighboring surface features. The height of solar panels is usually determined by the sum of a height of supports and elevation at a specific geographic location. In general, while elevation at the specific geographic location may change, the height of solar panels and supports should remain the same. 

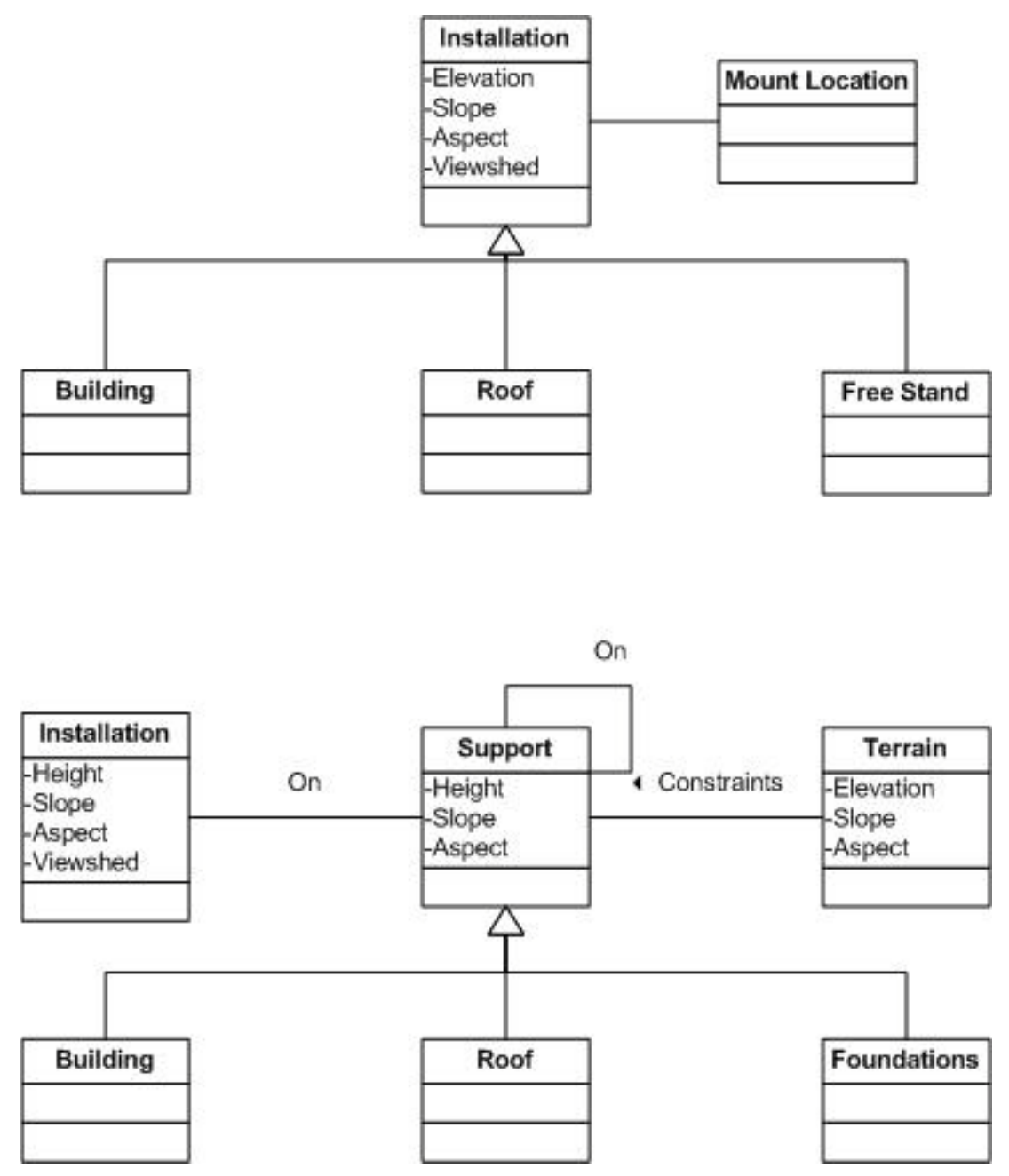

Figure 4-3: Solar Potential Installation Sites

To summarize, the conceptual data models illustrate the foremost entities of this project: the sun, obstructions, installation, and terrain. The models also represent key associations between the entities pertaining to this project. Due to the different degrees of obstructions, solar energy reaches the Earth's surface as either direct, diffused, or reflected solar radiations (ESRI, 2010d). Therefore, the amount of incoming solar radiation can vary at different geographic locations. Of all the potential installation sites, this project focused on rooftops of buildings and calculated the amount of incoming solar radiation. Based on these conceptual data models, the next section will examine how the project was implemented considering these entities and associations.

\subsection{Logical Data Model}

Logical data models enable the implementation of the entities and associations represented by the conceptual data models. Tomlinson (2007) states, "[a logical data] model not only represents the data in computer logic but also describes the data in terms 
the computer can virtually 'understand' - the model sets up its version of the real world with all its rules and orders that your data must follow” (pp. 93-94).

In the context of this project, a logical data model was created to describe four primary feature classes. The PointFileInformation feature class is a polygon feature class that contains information about LAS files. The RooftopOutlines feature class is a polygon feature class used to intersect output data and obtain relevant information about each building. The SampleLocations feature class is a single part point feature class generated from LiDAR point clouds to determine locations for the solar radiation calculation. The GlobalSolarRadiation feature class is a point feature class that contains results of the solar potential calculation for the given locations. Figure 4-4 represents associated attribute fields that are stored in an ArcGIS file geodatabase for the PointFileInformation, RooftopOutlines, SampleLocations, and GlobalSolarRadiation feature classes.

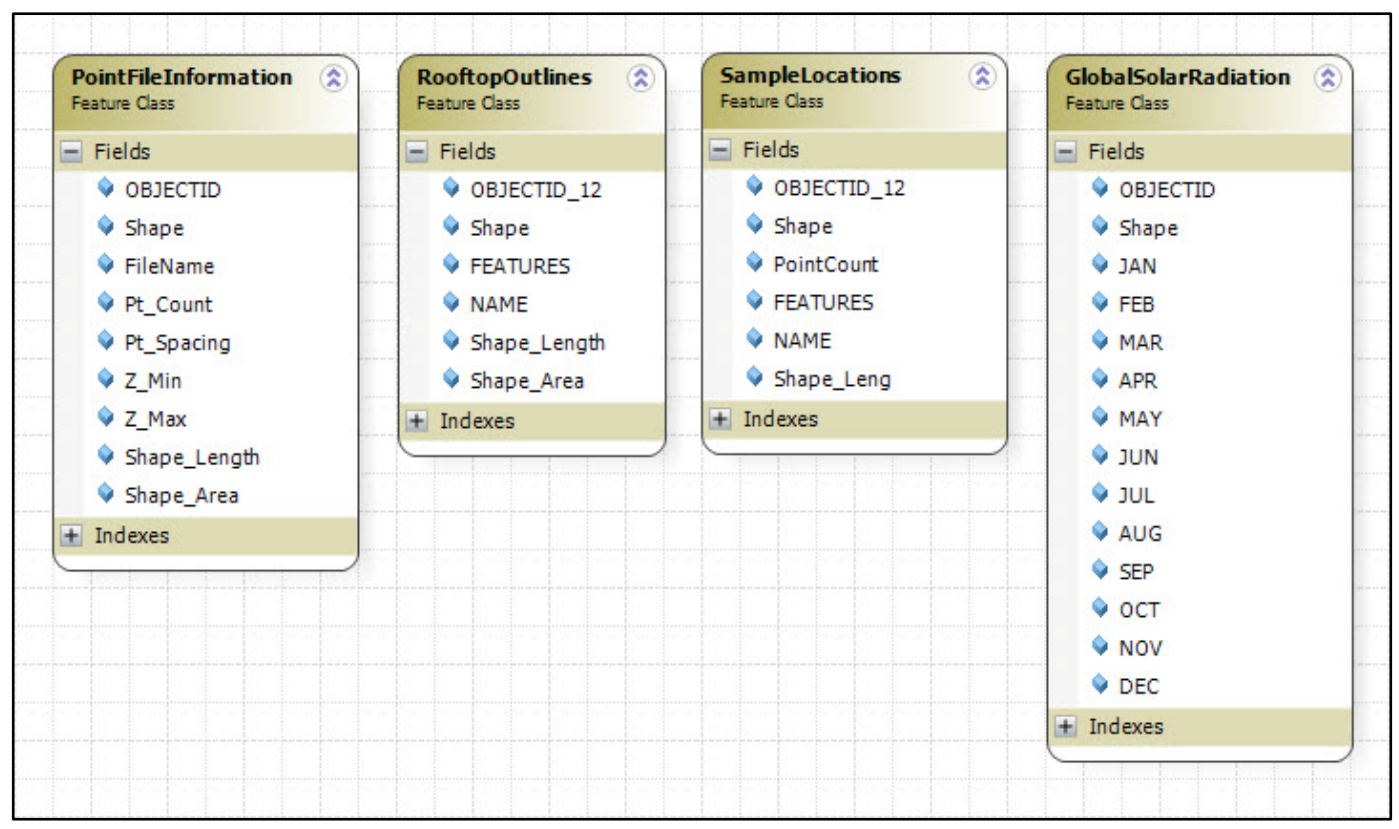

Figure 4-4: Project Database Layers

The PointFileInformation feature class includes information about LAS files. The SampleLocations feature class contains information about locations for the solar potential calculation. These points were generated from the LiDAR point clouds to use in the solar radiation analysis. All 73,158 point features were retained throughout the solar potential calculation and then the results of the calculation were added to each point feature and a new feature class, GlobalSolarRadiation, was created to store monthly solar potential values in kilowatt hour (kWh). The RooftopOutlines feature class contains NAME and Shape_Area fields displayed through the Web application. 


\subsection{Data Sources}

LiDAR data were the most crucial input for this solar radiation analysis. In the fall of 2009, an aerial survey using an airborne LiDAR scanner was conducted in the County of San Bernardino. This aerial survey was intended to capture LiDAR data and near-infrared and natural color digital images for the entire county. The data were provided in the NAD 1983 UTM Zone $11 \mathrm{~N}$ coordinate system. The density of LiDAR points is 2.01 points per square meters, and it can be used to generate 1-foot contours. The LiDAR data provided in LAS files were classified into six classes based on the following class codes: 1 for buildings and unclassified surface features, 2 for bare earth ground, 4 for trees and shrubs, 7 for errors that were measured below the ground level, 9 for water surfaces (rivers $>13$ ' wide and ponds $>20$ ' diameter), and 12 for overlaps (over $30 \%$, actually vary from $40-80 \%)$.

This project also needed building footprint data of the study area with a polygon feature class. The polygon feature class was provided by the Redlands Institute and stored in the NAD83 California State Plane Zone 5 coordinate system. This feature class contains 114 polygons. Although it was not directly involved in the solar radiation analysis, this polygon feature class was used to spatially extract multipoint features that were classified as buildings and spatial interpolation results by using the Inverse Distance Weighted (IDW) method.

\subsection{Data Scrubbing and Loading}

The solar radiation analysis in this project required LiDAR data with LAS files. Prior to using the LiDAR data as primary input, the LAS files were examined by using the Point File Information tool in the 3D Analyst toolbox. Figure 4-5 shows the summary of the four LAS files' information. The Point Spacing field shows a one-dimensional measurement of points along a line (ESRI, 2010c). Based on these four point spacing values, average point spacing value was determined to be 0.69 meters. This average point spacing value was required in the LAS to Multipoint tool in the 3D Analyst toolbox and was also used to approximate a cell size for output raster datasets.

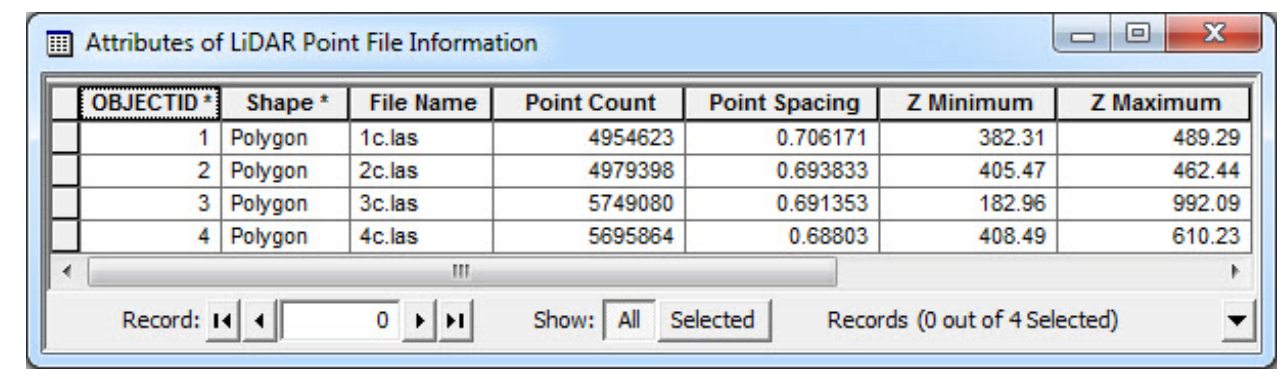

\section{Figure 4-5: Point Spacing Information for Each LAS File}

Before loading the building feature class to the database, the polygon feature class was examined and prepared. This polygon feature class is used by various departments across the university campus and is updated when necessary. Since this feature class 
contains not only buildings but also all other facilities located on the campus, polygon features that indicate buildings were first selected using the FEATURES field. An SQL of FEATURES = 'building' was used to select 118 buildings' polygon features in the study area. A new layer file was created from the 118 polygon features and was exported to a file geodatabase feature class. Four polygon features that indicate a building, "Village Apartments," were deleted from the feature class because this building does not exist today. One polygon feature that indicates a swimming pool, "Thompson Aquatic," was modified in order to eliminate the water surface features where a density of LiDAR point data is very low. This feature class was used when extracting the multipoint features within the rooftop outlines. It was also important to change the coordinate system of this polygon feature class. After the layer file was exported to a file geodatabase feature class, the coordinate system was changed from NAD 1983 StatePlane California V FIPS 0405 Feet to NAD 1983 UTM Zone 11N by using the Project tool in the Data Management toolbox.

In addition to changing the projection and extracting only features that indicate buildings, various attribute fields of the polygon feature class were modified in order to improve the geodatabase's performance level. OBJECTID is essentially managed by ArcGIS. First, this polygon feature class uses the OBJECTID_12* field, which was created when the feature class was loaded to the geodatabase, and was used as a unique identifier to conduct the attribute index. The attributes of the point feature class contain redundant fields such as OBJECTID, FID_UoR_Campus2007, and OBJECTID_1*. More importantly, these fields except OBJECTID_1* included blank records. These fields were removed from the geodatabase because these situations made it hard for users to identify data in an efficient manner. The polygon feature class also contained unnecessary fields for the solar radiation analysis. For example, WALKWAY, ROAD, GRASS, OTHER, and BUILDING fields were regarded as entirely unnecessary.

\subsection{Summary}

This chapter examined four key components of database design. The conceptual data models illustrated the foremost entities of this rooftop solar radiation analysis. They include the sun, obstruction, installation, and terrain. The models also represented important associations between the entities pertaining to this project. The logical data model was then created in order to implement the entities and associations represented by the conceptual data models. Prior to this analysis, these primary inputs were examined for content and modified to meet the needs of the solar radiation analysis. For the LiDAR data, the average point spacing value was determined and used to approximate an output cell size throughout the analysis. For the building footprint data, a coordinate system was first changed, and unnecessary attribute data were removed. 


\section{Chapter 5 - Implementation}

Chapter 5 discusses the project implementation, which includes generating primary inputs for solar radiation analysis, calculating the amount of solar radiation, creating solar radiation surface using a spatial interpolation technique, and displaying the analysis results on the Web. The first three tasks were completed by creating the Solar Radiation Analysis model using the ArcGIS ModelBuilder. The last task was fulfilled by creating a Web application using the ArcGIS API for JavaScript (JavaScript API) development framework.

\subsection{Primary Inputs}

The Solar Radiation Analysis model was created by using the ArcGIS ModelBuilder in order to perform a sequence of algorithms. This model first required a DSM and a point feature class as the primary inputs of the Points Solar Radiation tool. The following subsections describe how to generate a DSM and a point feature class from LiDAR point clouds. Figure 5-1 shows the overall workflow for generating these primary inputs.

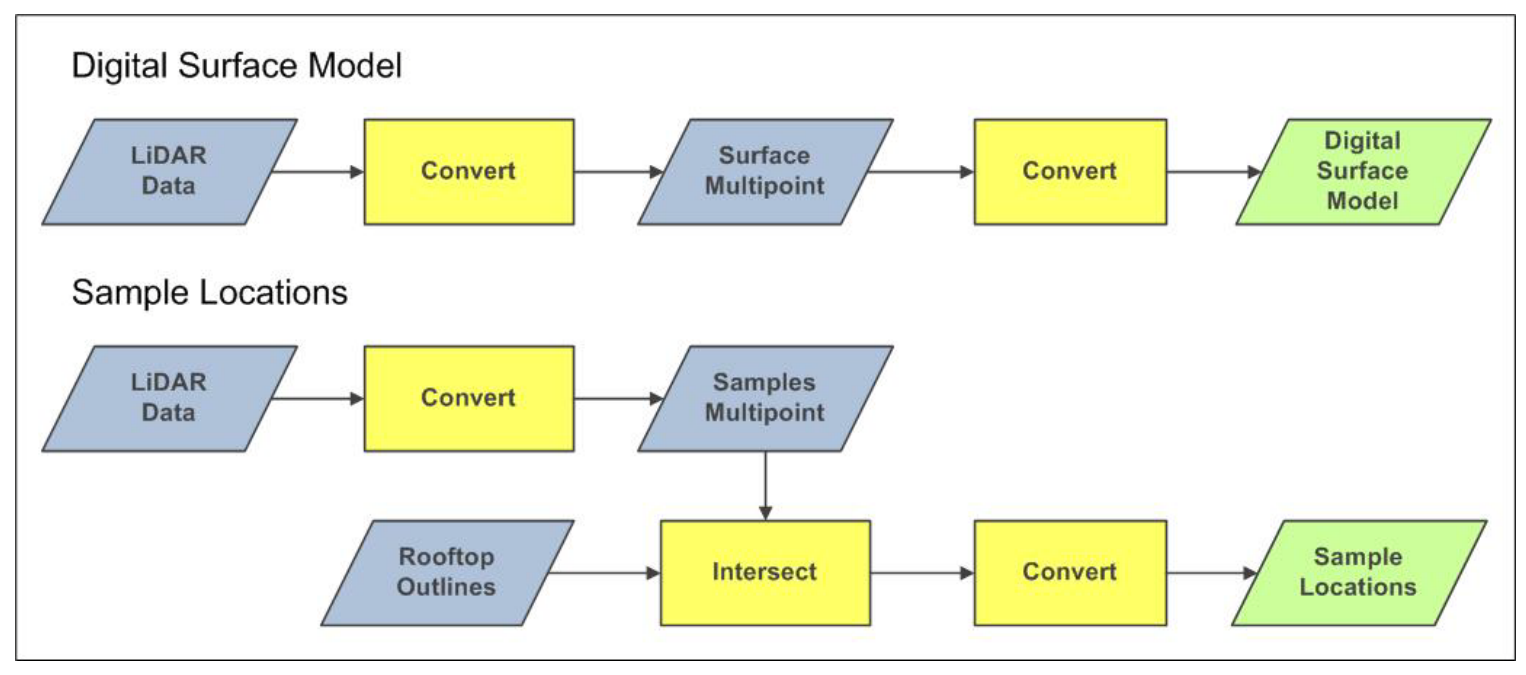

Figure 5-1: Overall Workflow for Generating Primary Inputs

\subsubsection{Generating DSM}

The DSM of the University of Redlands campus was generated from the LiDAR point clouds. The LAS to Multipoint tool in the 3D Analyst toolbox was first used to convert LAS files to a multipoint feature class. This tool can accept one or more LAS files as input, and one single multipoint feature class was created. Multipoint features are useful because thousands of points can be stored in one database row to reduce the total number of rows in a feature class table, which improves data processing efficiency (ESRI, 2010c). Four LAS files that cover the entire study area were selected as input files, which included buildings and unclassified features (class code of 1), bare earth ground (class code of 2), trees and shrubs (class code of 4), and overlaps between LiDAR strips (class 
code of 12). The average spacing value of 0.69 , which refers to one-dimensional measurement of points along a line, was defined to be the mean LiDAR point spacing of the four LAS files (Figure 5-2). At the same time, the projection was set to NAD 1983 UTM Zone 11 North, and the Z coordinate system was set to NAVD_1988.

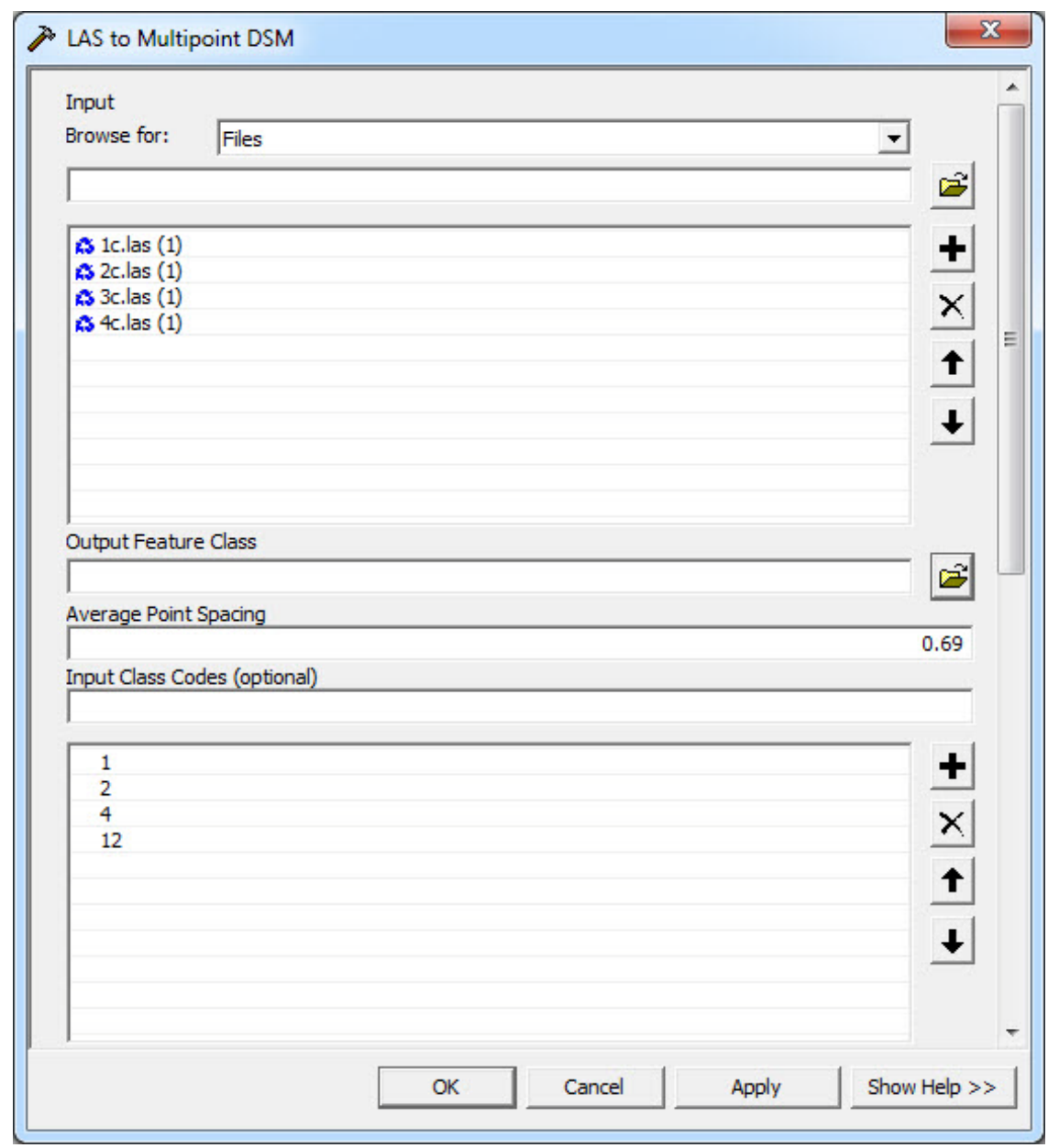

Figure 5-2: LAS to Multipoint Tool Interface for DSM

Once the multipoint feature class was obtained, it was further converted to a raster dataset using the Feature to Raster tool in the Conversion toolbox. Due to the existence of areas with a low LiDAR point density and the exclusion of LiDAR point clouds that are classified as errors (class code of 7) and water surfaces (class code of 9), it is possible that output cells of the raster dataset may contain no elevation values, depending on the output cell size. In order to reduce the number of null values, the cell size of the raster dataset was defined as 2 meters (Figure 5-3). 


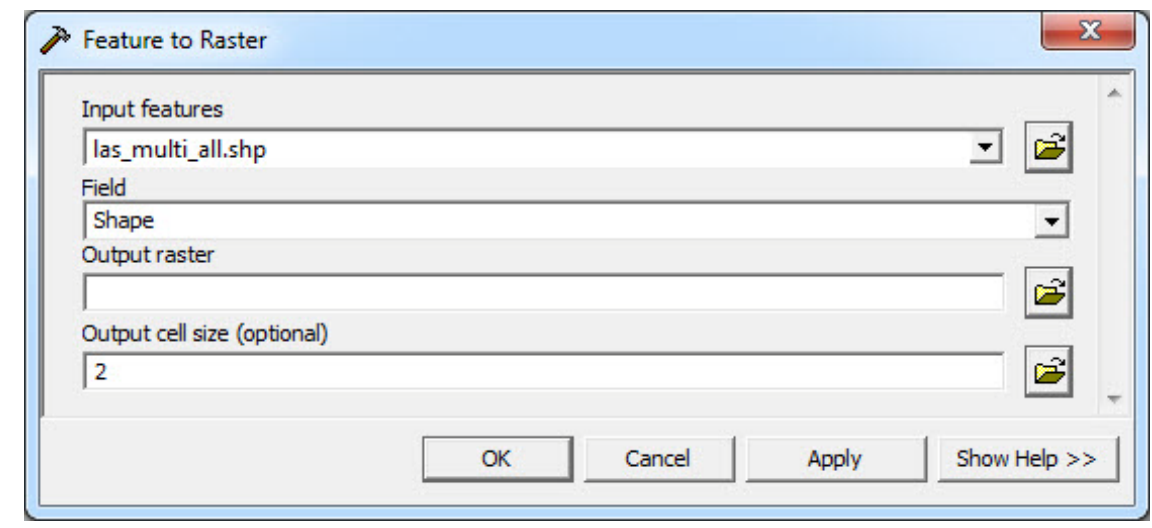

Figure 5-3: Feature to Raster Tool Interface and Key Parameters

\subsubsection{Generating Point Feature Class}

A point feature class, which indicates the sample locations, is another primary input of the Points Solar Radiation tool. In this project, the LiDAR point clouds of the building rooftops were used as the sample locations. Unlike generating the DSM, only points that were classified with a class code of 1 , which refers to points for buildings and unclassified surface features, were converted to a multipoint feature class by using the LAS to Multipoint tool (Figure 5-4). Therefore, the resulting multipoint feature class contains both building rooftops and other unclassified areas. 


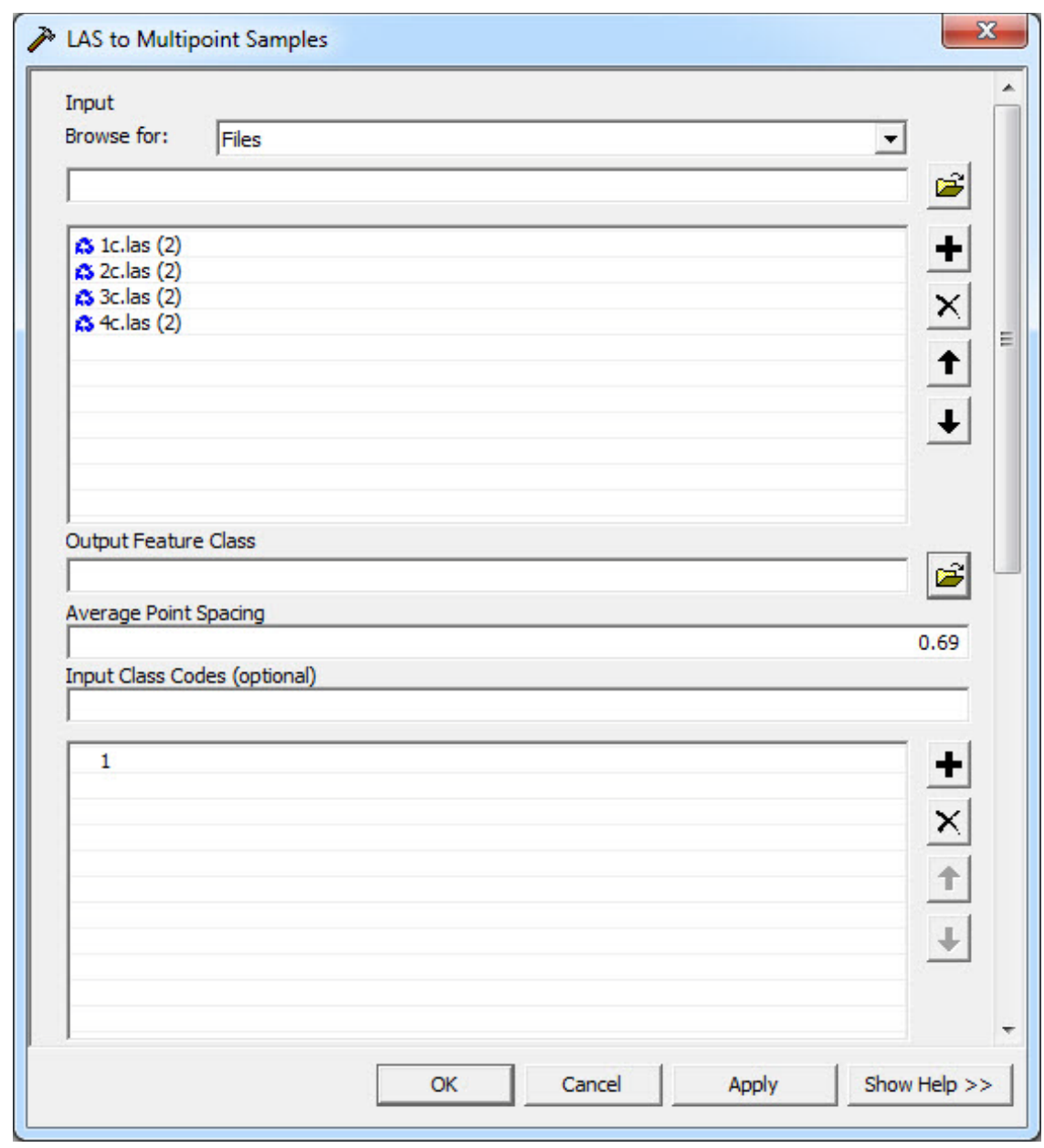

Figure 5-4: LAS to Multipoint Tool Interface for Sample Points

To extract points within the rooftop boundaries, the multipoint feature class was intersected with the building rooftop polygon feature class using the Intersect tool in the Analysis toolbox. The multipart point features were then separated to singlepart point features while maintaining the original attributes. As a result, the number of rows in the feature class increased from 198 to 73,158, with each record representing a single point falling on the rooftops (Figure 5-5). The Points Solar Radiation tool required separate singlepart point features as primary inputs. 


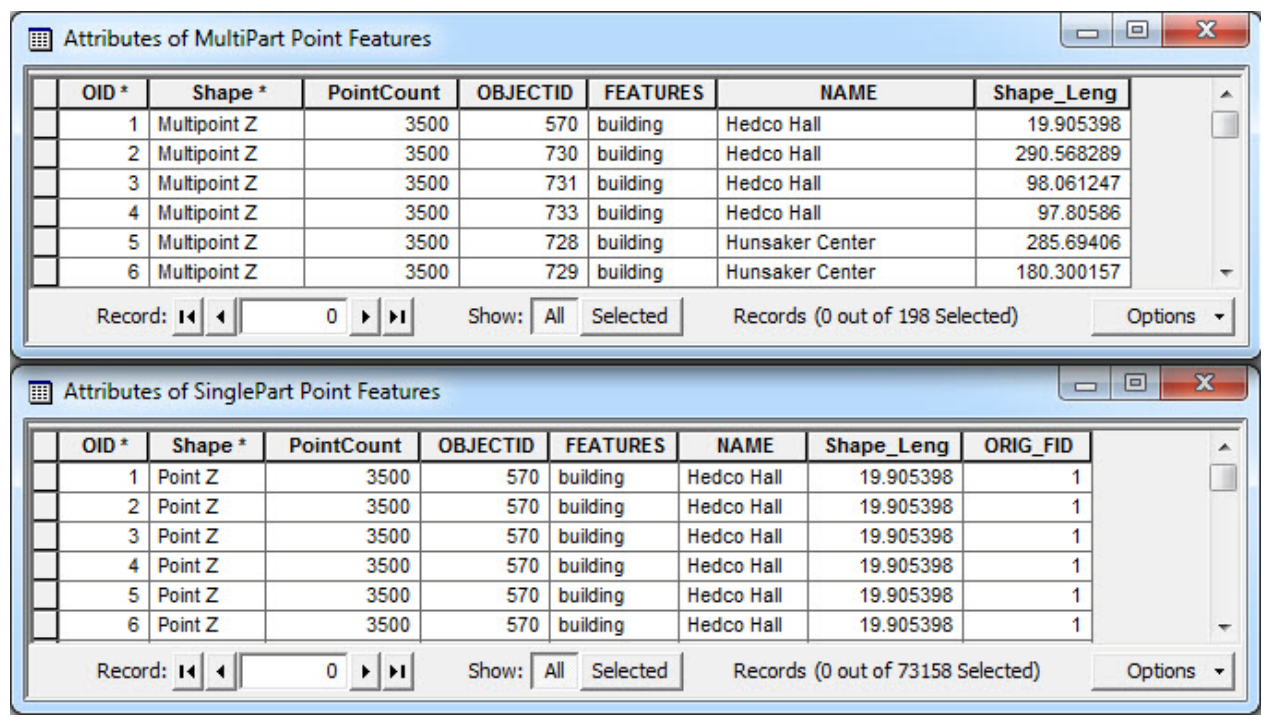

Figure 5-5: Exploding Multipart Feature (Top) to Singlepart Feature (Bottom)

\subsection{Solar Radiation Analysis}

Using the DSM and sample locations point feature class as primary inputs, the Points Solar Radiation tool calculated the amount of radiant energy for given locations that are stored in the point features. The Points Solar Radiation tool, which requires a DEM as a primary input, analyzes and visualizes the effects of the sun for given locations for specific time periods. This tool accounts for various factors such as atmospheric conditions, latitude, elevation, surface orientation including slope and aspect, the sun's angle, and shadows cast by surrounding surface features (ESRI, 2010e). Figure 5-6 indicates the overall workflow for calculating the amount of solar radiation and creating raster datasets using a spatial interpolation technique.

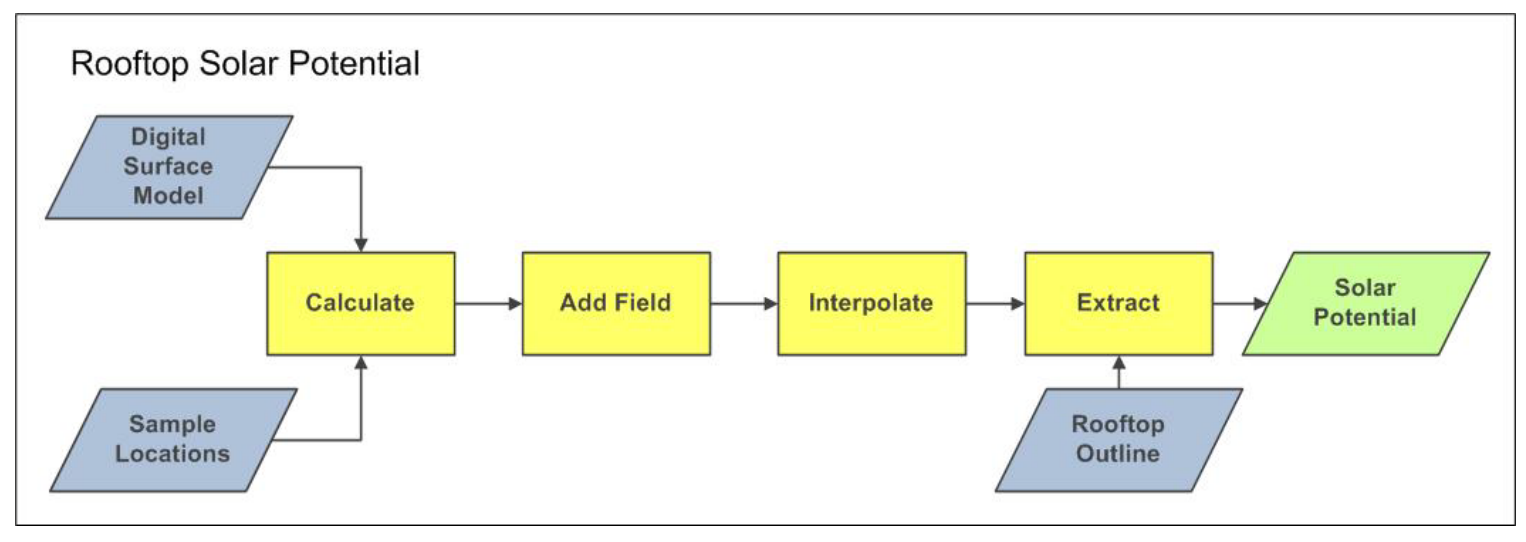

Figure 5-6: Overall Workflow for Calculating Solar Radiation 


\subsubsection{Generating Point Feature Class}

There are several key parameters to enter when using the Point Solar Radiation tool (Figure 5-7). First, latitude was set to 34.07 at which the study area is located. Time configuration was also set to "Whole year with monthly interval" to calculate the amount of solar radiation by month for the year 2009. The option for creating outputs for each interval was also checked. In addition to these two key parameters, sky size/resolution, which is the resolution or sky size for the viewshed, skymap, and sunmap grids (units: cells), were set to 1,024 . The skymap represents "a hemispherical view of the entire sky divided into a series of sky sectors defined by zenith and azimuth angles” (ESRI, 2010d) and is used as a reference map to calculate diffuse solar radiation for a particular location (ESRI, 2010d). Similarly, the sunmap consists of "discrete sunmap sectors defined by the sun's position at particular intervals during the day and time of year" (ESRI, 2010d) and is used as a reference map to calculate direct solar radiation for a particular location (ESRI, 2010d). The increasing computing efficiency makes it possible to select the higher number for the sky size/resolution and to generate more accurate results of global solar radiation calculation. 


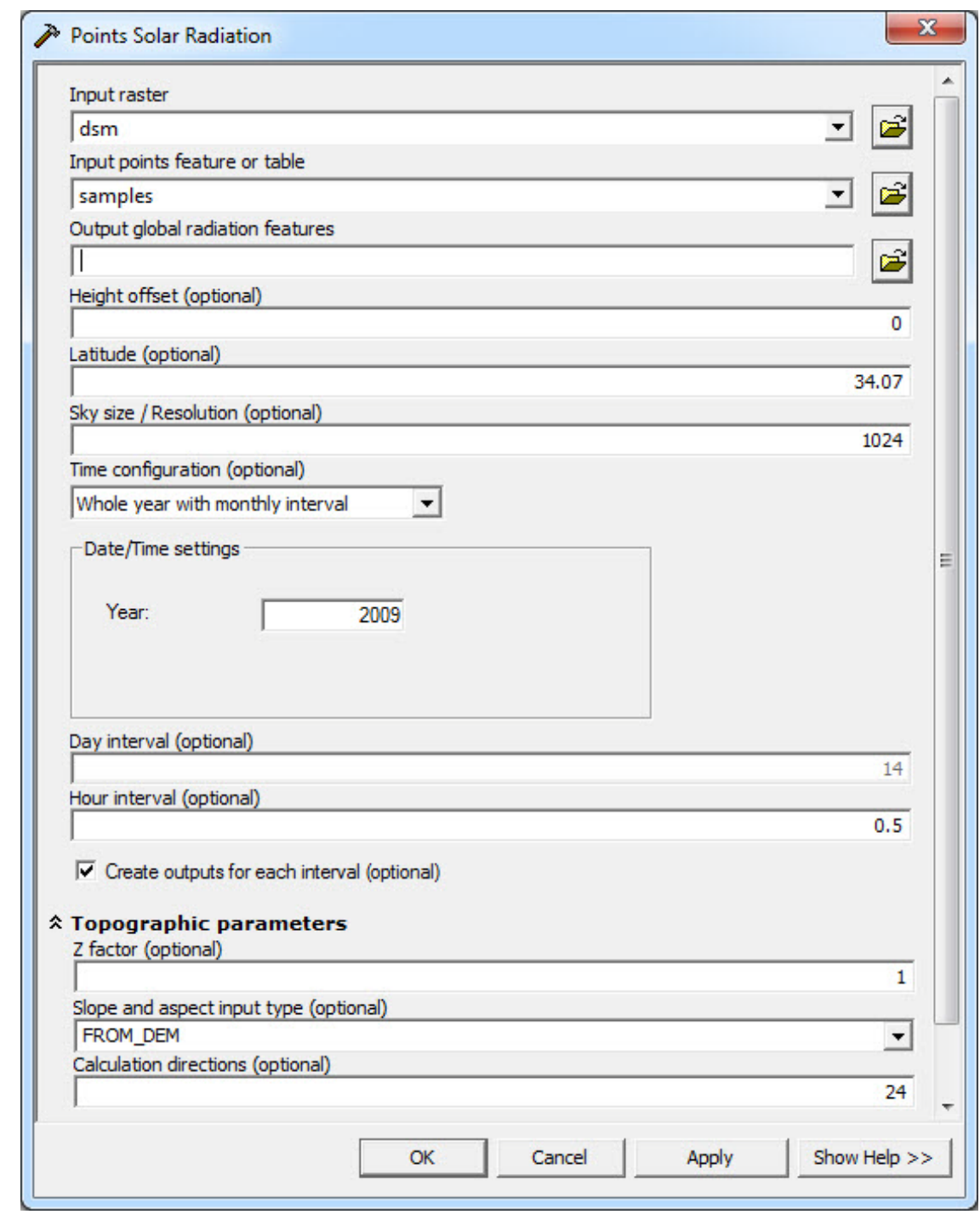

Figure 5-7: Points Solar Radiation Tool Interface and Key Parameters

At the same time, the number of calculation directions, which is one of topographic parameters, was also adjusted to 24. This is the number of azimuth directions that are used when measuring a horizon angle that determines the maximum angle of sky obstruction and then calculating the viewshed (ESRI, 2010d). Horizon angles at unspecified directions are interpolated based on the angles at the specified number of azimuth directions. Therefore, the higher number of azimuth directions can potentially result in the more accurate viewshed for the particular location (ESRI, 2010d). The default value was 32 directions, which is considered to be an adequate value for complex topography (ESRI, 2010f). Although the study area can be considered as relatively flat, a value of 24 was chosen for increased computing efficiency. In conjunction with overlaying sunmap and skymap, the higher number of azimuth directions will potentially generate more accurate results of the viewshed analysis. 
After key parameters of the solar radiation analysis were defined, the Points Solar Radiation tool was run. This tool can calculate three types of solar radiations: direct radiation, which refers to those intercepted unimpeded; diffused radiation, which refers to those scattered by atmospheric constituents; and global radiation, which refers to the sum of direct and diffused radiations (ESRI, 2010d). From these three output feature classes, the global radiation feature class as an output of the analysis was selected for this project. The output point feature class contains twelve new fields that ranged from T0 to T11, in which solar potential values of each month are stored in watt hour (Wh). In order to convert the measurement units to kWh, twelve new fields with the data type of DOUBLE were added to the output global radiation feature class by the Add Field tool in the Data Management toolbox. These new fields were named to JAN, FEB, MAR, APR, MAY, JUN, JUL, AUG, SEP, OCT, NOV, and DEC. The original solar radiations were then converted to kWh by the Calculate Field tool in the Data Management toolbox. The solar potential values in Wh were divided by 1,000 (Figure 5-8).

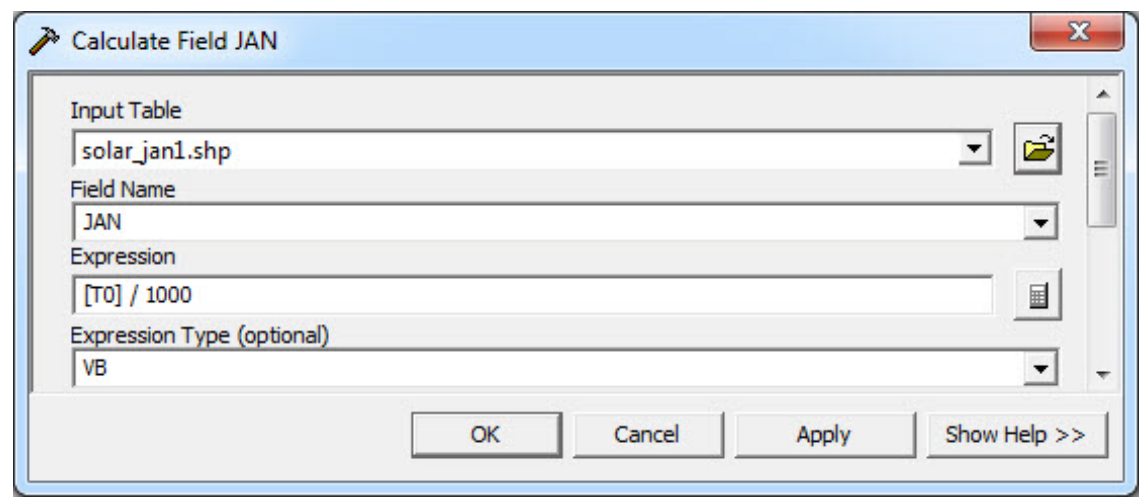

Figure 5-8: Calculate Field Tool Interface and Expression

\subsubsection{Interpolating Solar Potential}

After the global solar radiation was calculated for the sample points on building rooftops, a spatial interpolation method was applied to estimate those areas that did not have measurements. In doing so, the discrete point output became a continuous solar energy surface covering entire rooftops. This project used the Inverse Distance Weighted (IDW) tool in the Spatial Analyst toolbox to conduct interpolation analysis. As shown in Figure 5-9, a search radius distance, which restricts input sample points, was set to 3 meters, and each output cell size was set to 2 meters to match the input DSM cell size. The search radius distance was defined as 3 meters to avoid cells with no data while eliminating the influence from points in neighboring buildings. Further, because the extent of output raster datasets are not necessarily in line with building boundaries, the output raster datasets from the IDW tool were extracted by the rooftop outline polygon features using the Extract by Mask tool in the Spatial Analyst toolbox. 


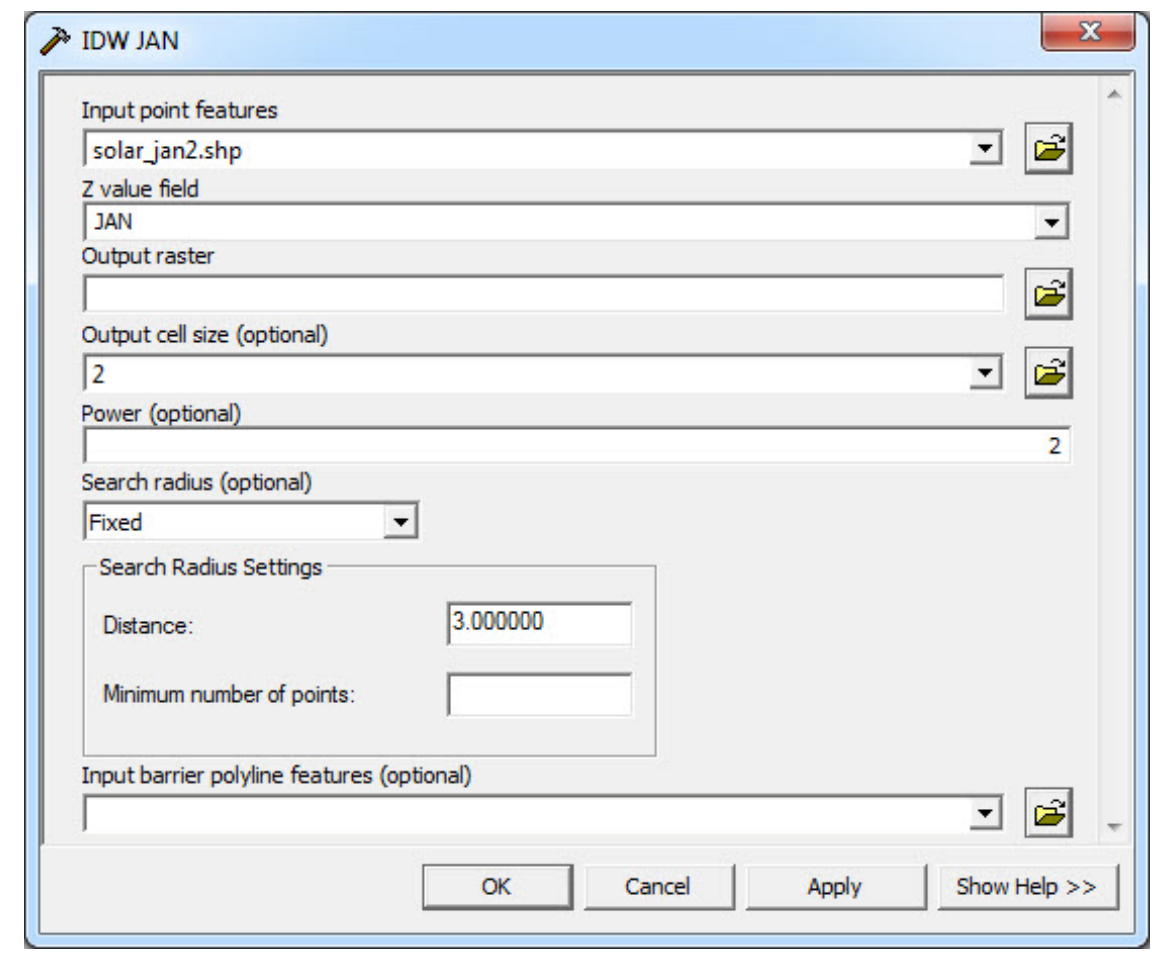

\section{Figure 5-9: IDW Tool with 3m Search Radius}

\subsection{Web Mapping Application}

The last major component of this project was to create a Web mapping application. During this phase of the project, several attempts were made to test which development framework was effective. Prior to the JavaScript API Web application, the ArcGIS Server Web ADF development framework was used to display solar potential maps and calculate the solar potential values at a location of interest on-the-fly. Based on lessons learned from this first attempt, the JavaScript API mapping application was designed to meet the initial project requirements and use different functionalities of the Web application.

JavaScript API is "a browser-based API for developing high performance [and] easyto-use mapping applications" (ESRI, 2010g). Because this "runs immediately inside [a] browser," users do not need to wait for responses from the server (ESRI, 2010g). In order to complete this task, desired map services were first published to ArcGIS Server. Three major functions, including identifying a target rooftop, calculating the amount of solar potential, and displaying the analysis results in chart and table form were then created by using JavaScript API.

\subsubsection{Publishing a Map Service Using ArcCatalog}

A map document that contained the Sample Locations and Rooftop Outlines layers was first created in ArcMap (Figure 5-10). Prior to saving the ArcMap document as 2009GlobalSolarRadiation.mxd file, a coordinate system of the data frame was set to 
WGS 1984 Web Mercator (Auxiliary Sphere) because the world imagery map that would be added from ArcGIS Online later is based on the WGS 1984 Web Mercator (Auxiliary Sphere) coordinate system, while these shapefiles are based on the NAD 1983 UTM Zone 11 North coordinate system. At the same time, the number of decimal places for solar potential values in the JAN, FEB, MAR, APR, MAR, JUN, JUL, AUG, SEP, OCT, NOV, and DEC fields of the Global Solar Radiation layer were decreased to " 1 " in order to keep the table consistent and effectively display the analysis results.

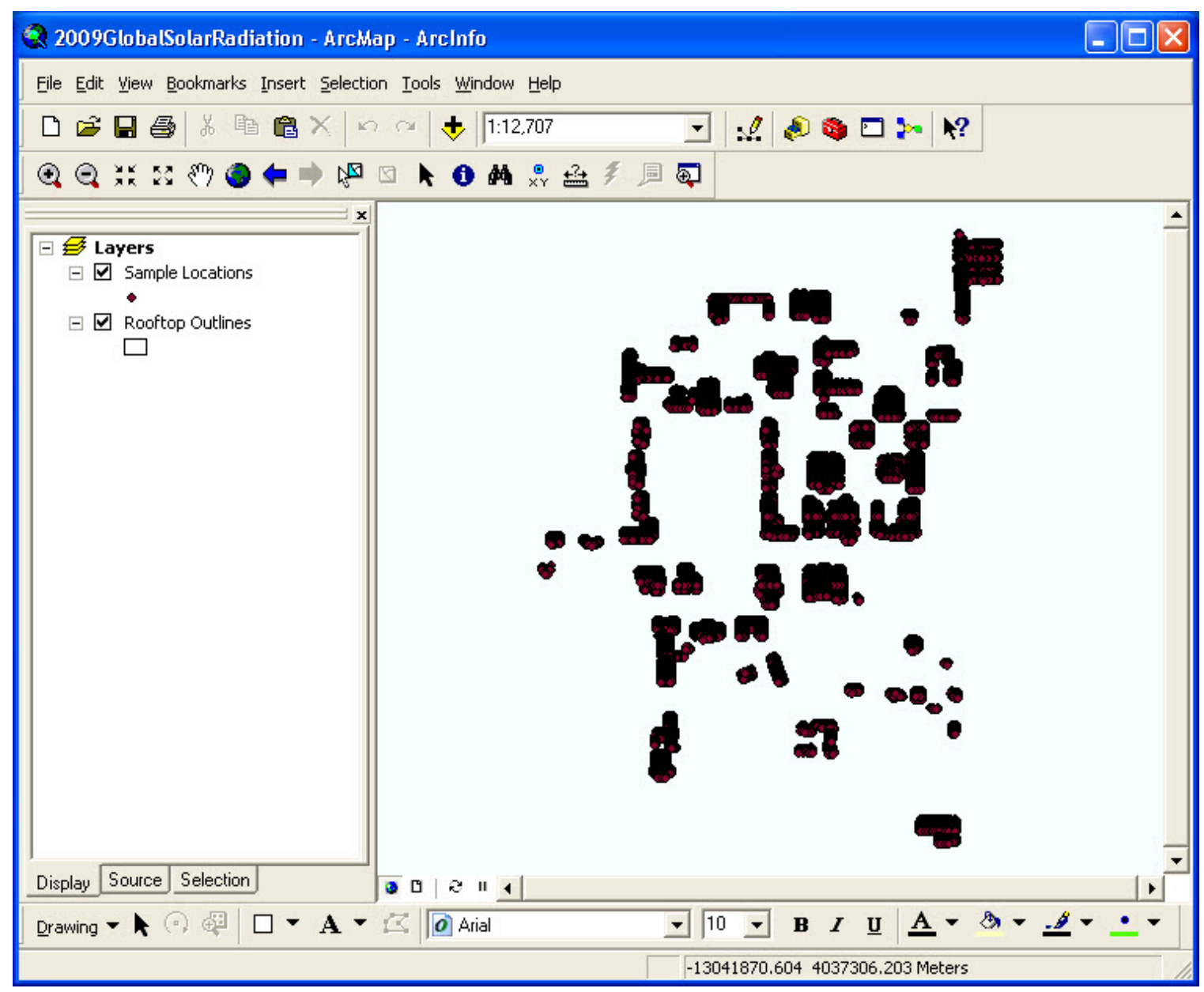

Figure 5-10: 2009GlobalSolarRadiation ArcMap Document Overview

In the same manner, another map document containing twelve layers that include monthly solar potential values in raster format was also created in ArcMap (Figure 5-11). Using the WGS 1984 Web Mercator (Auxiliary Sphere) coordinate system, this ArcMap document was saved as 2009MonthlySolarPotentials.mxd. 


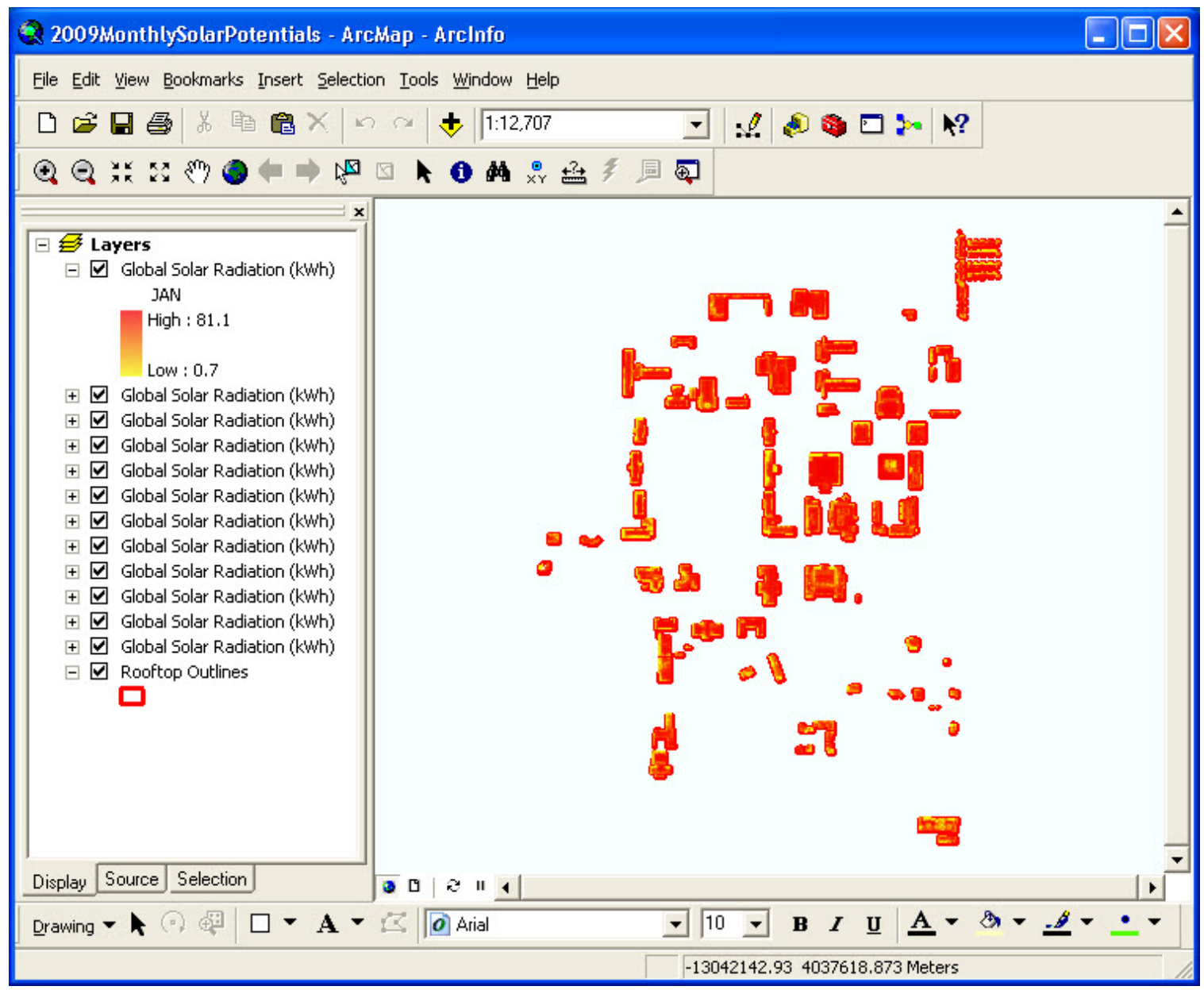

Figure 5-11: 2009MonthlySolarlPotentials ArcMap Document Overview

These map documents were then published to an ArcGIS Server by using the Publish to ArcGIS Server wizard in ArcCatalog. Once the publishing task was completed, the map documents were added to a list of map services in the ArcGIS Services Directory. For the 2009GlobalSolarRadiation map service, the spatial reference system was “102100,” which refers to the WGS 1984 Web Mercator (Auxiliary Sphere) coordinate system, and unique IDs of " 0 ” and " 1 ” were assigned to the Sample Locations and Rooftop Outlines map layers, respectively (Figure 5-12). 


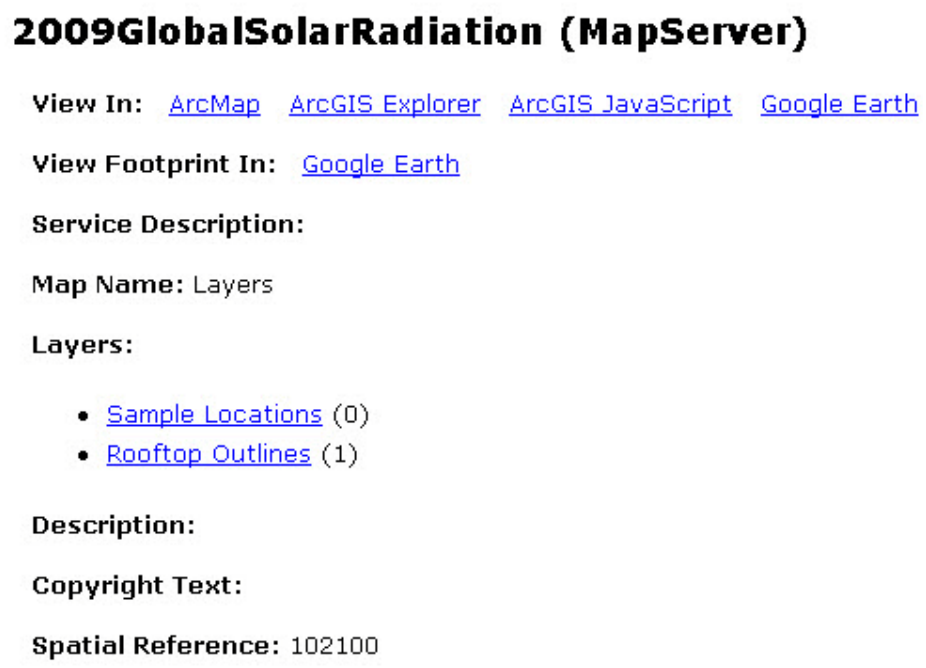

\section{Figure 5-12: 2009GlobalSolarRadiation Map Service Information}

In the same manner, for the 2009MonthlySolarPotentials map service, the spatial reference system was "102100" to adjust the map service to the WGS 1984 Web Mercator (Auxiliary Sphere) coordinate system, and twelve unique IDs ranging from "0" through " 11 " were assigned to twelve monthly solar potential map layers. A unique ID of “12” was assigned to the Rooftop Outlines map layer (Figure 5-13). 


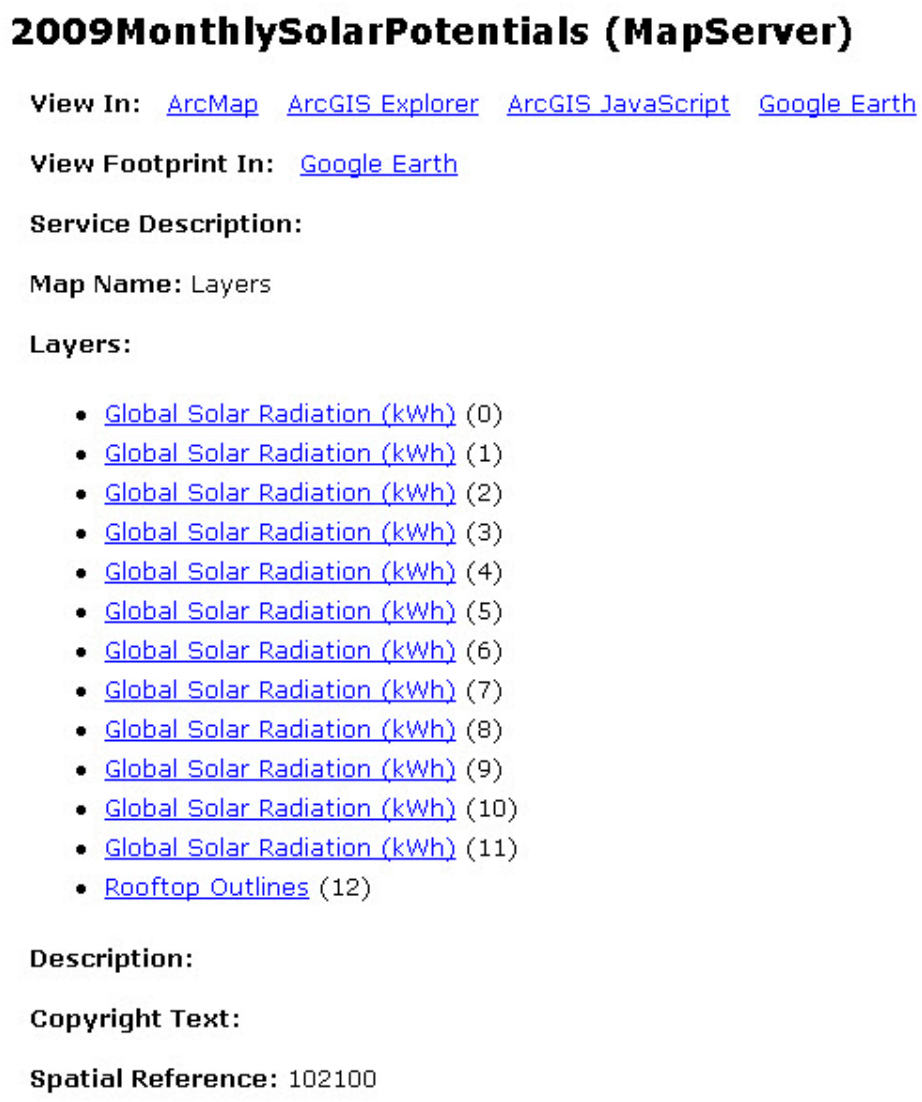

Figure 5-13: 2009MonthlySolarPotentials Map Service Information

In order to correctly overlay a tiling scheme of the map layers with the world imagery base map from ArcGIS Online that is already in the 102100 tiling scheme, the same tiling scheme was loaded from the conf102100.xml file to the 2009GlobalSolarRadiation map service only. The 2009MonthlySolarPotentials map service, on the other hand, needed to draw the map service dynamically from the data without loading the tiling scheme from the conf102100.xml file because this map service contains multiple map layers which visibility is toggled by a user (Figure 5-14). 


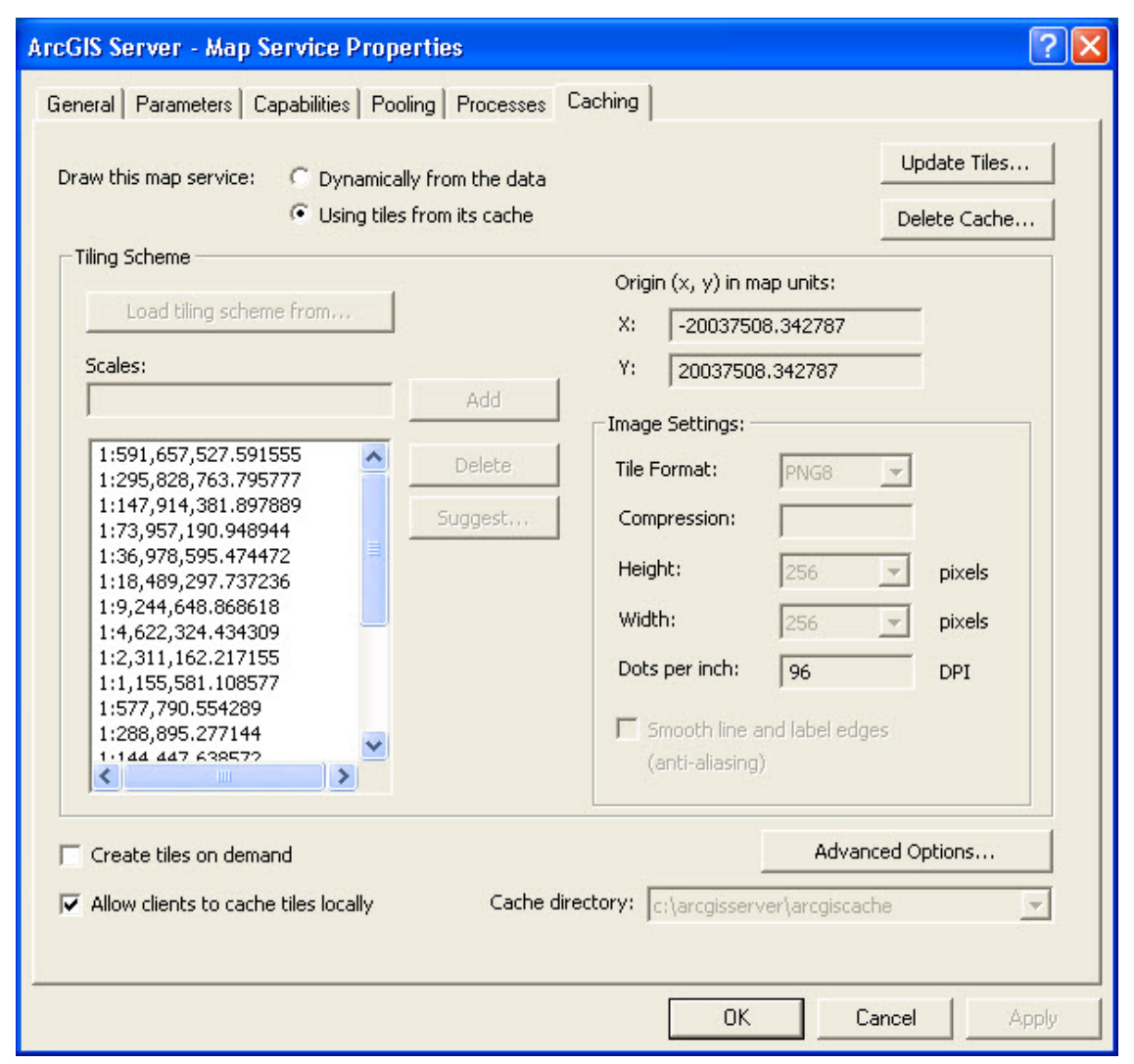

Figure 5-14: Selecting a Tiling Scheme for 2009GlobalSolarRadiation Map

For the purpose of displaying monthly solar potential maps using ArcGIS Server Web ADF, twelve separate map services were also created, and the previously discussed tiling scheme was added to these map services. This task was needed to toggle these map layers' visibility.

\subsubsection{Developing a Geoprocessing Service with ArcGIS Server Web ADF}

Prior to a Web application using JavaScript API, ArcGIS Server ADF application was created to display monthly solar potential maps and calculate solar potential for a given location on-the-fly. First, the previously discussed model was largely simplified by removing the generating primary inputs and conducting spatial interpolation tasks. At the same time, intermediate data were directed to a scratch workspace folder by adding \%scratchworkspace\% to each output field and a feature set was added to let users define a sample location for the calculation by drawing a polygon (Figure 5-15). These modifications were necessary in order to minimize the required processing time for displaying the analysis results. 


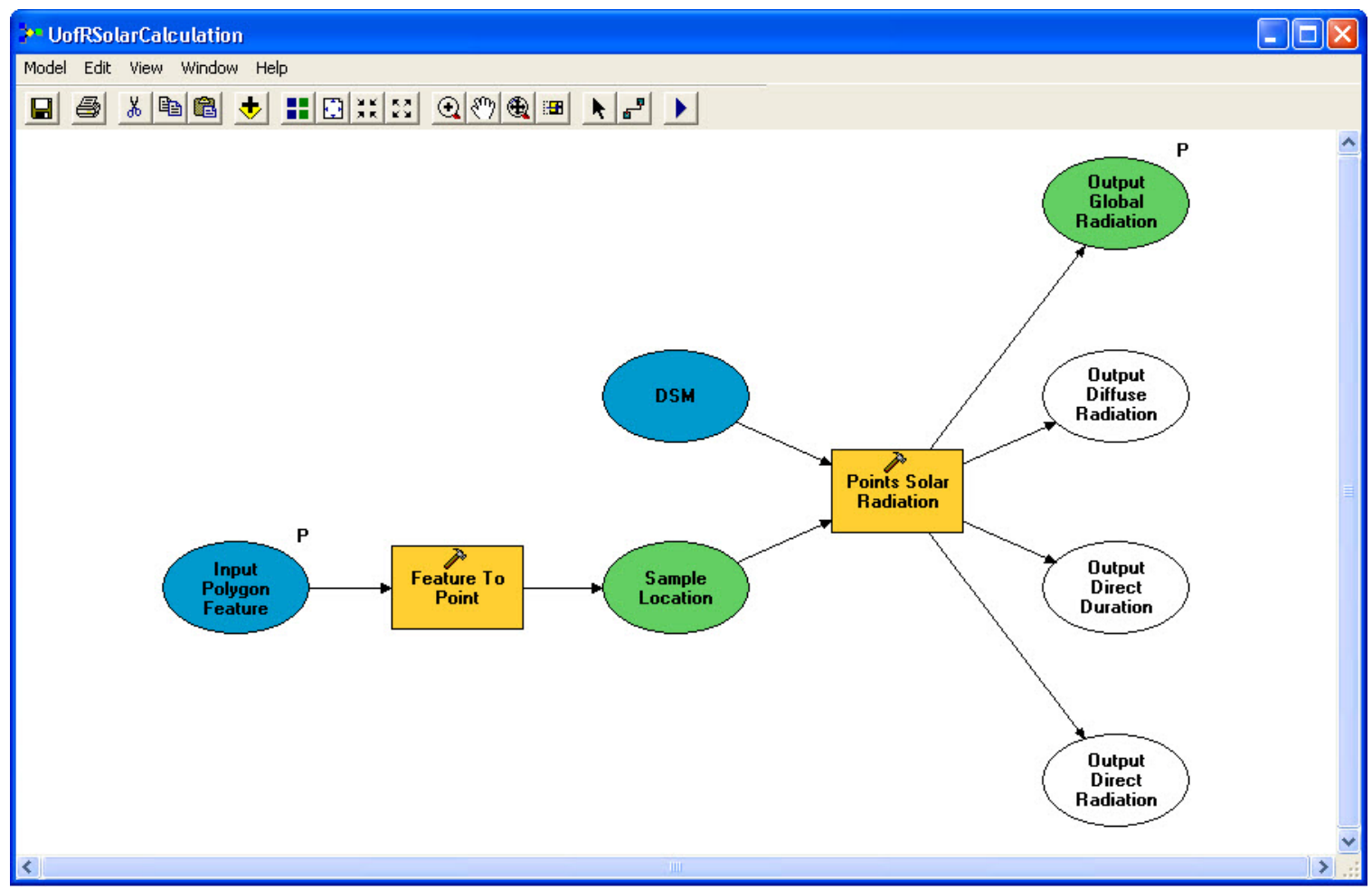

Figure 5-15: Simplified Model to Publish as Geoprocessing Service

Once the model was created, an ArcGIS toolbox that contains the model was published as a geoprocessing service by using the Publish to ArcGIS Server wizard in ArcCatalog. An execution type of this geoprocessing service was changed to "Synchronous" in the Parameters tab in the Geoprocessing Service Properties window (Figure 5-16). This execution type is "best suited for quick processes and small result sets [because] jobs can be executed immediately. Results are not saved on [the] server [but are] temporarily held on [the user's] machine” (ESRI, 2009, p. 6-14). 


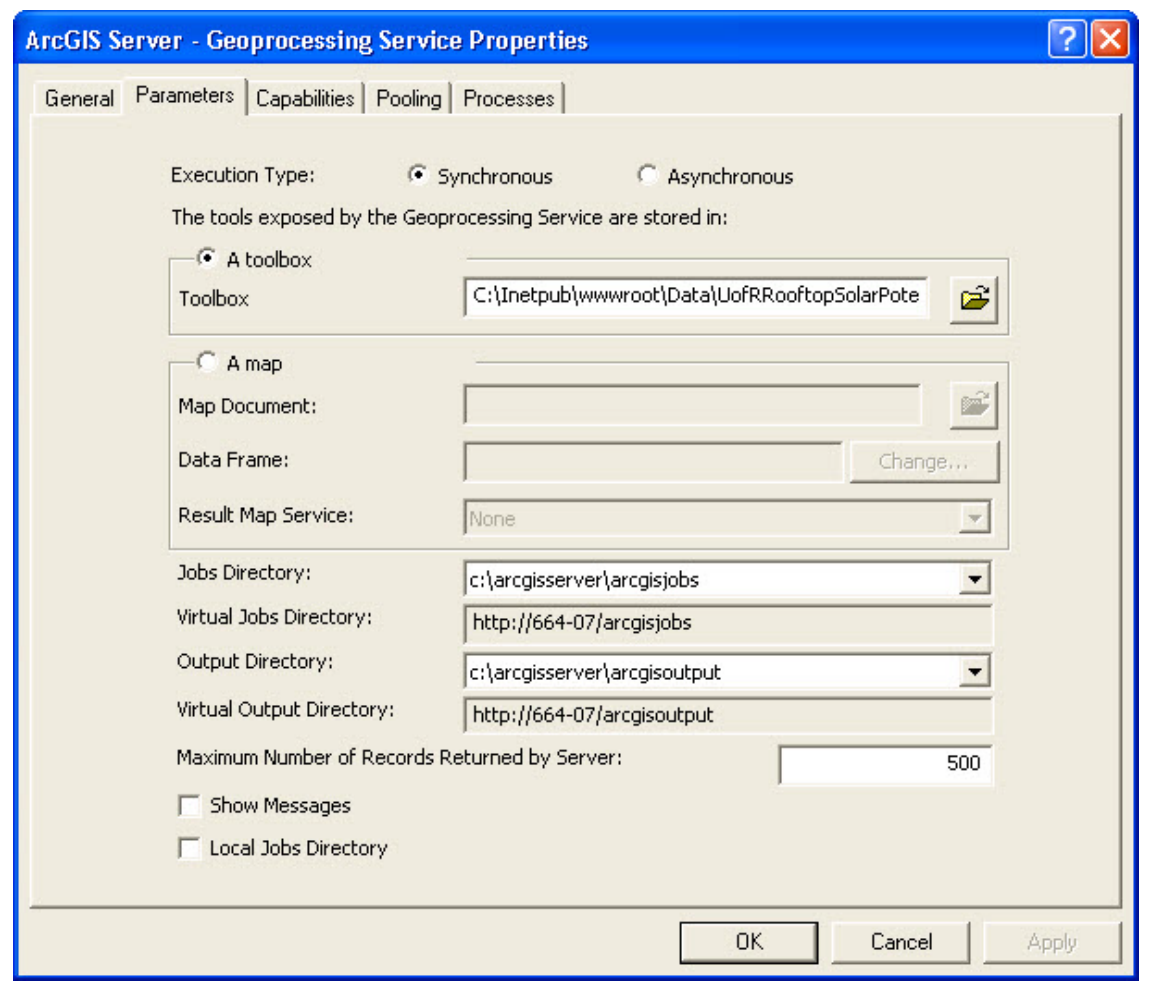

Figure 5-16: Selecting a Synchronous Execution Type

Once the new model was published as a geoprocessing service to the ArcGIS Server, a new Web application was created in the ArcGIS Server Manager. Desired map services, including the world imagery map from ArcGIS Online, twelve monthly solar potential, and the Rooftops map services from the ArcGIS Services Directory were added to the application under the Layers tab. At the same time, the default spatial extent was set to the map extent of the Rooftop map service (Figure 5-17). 


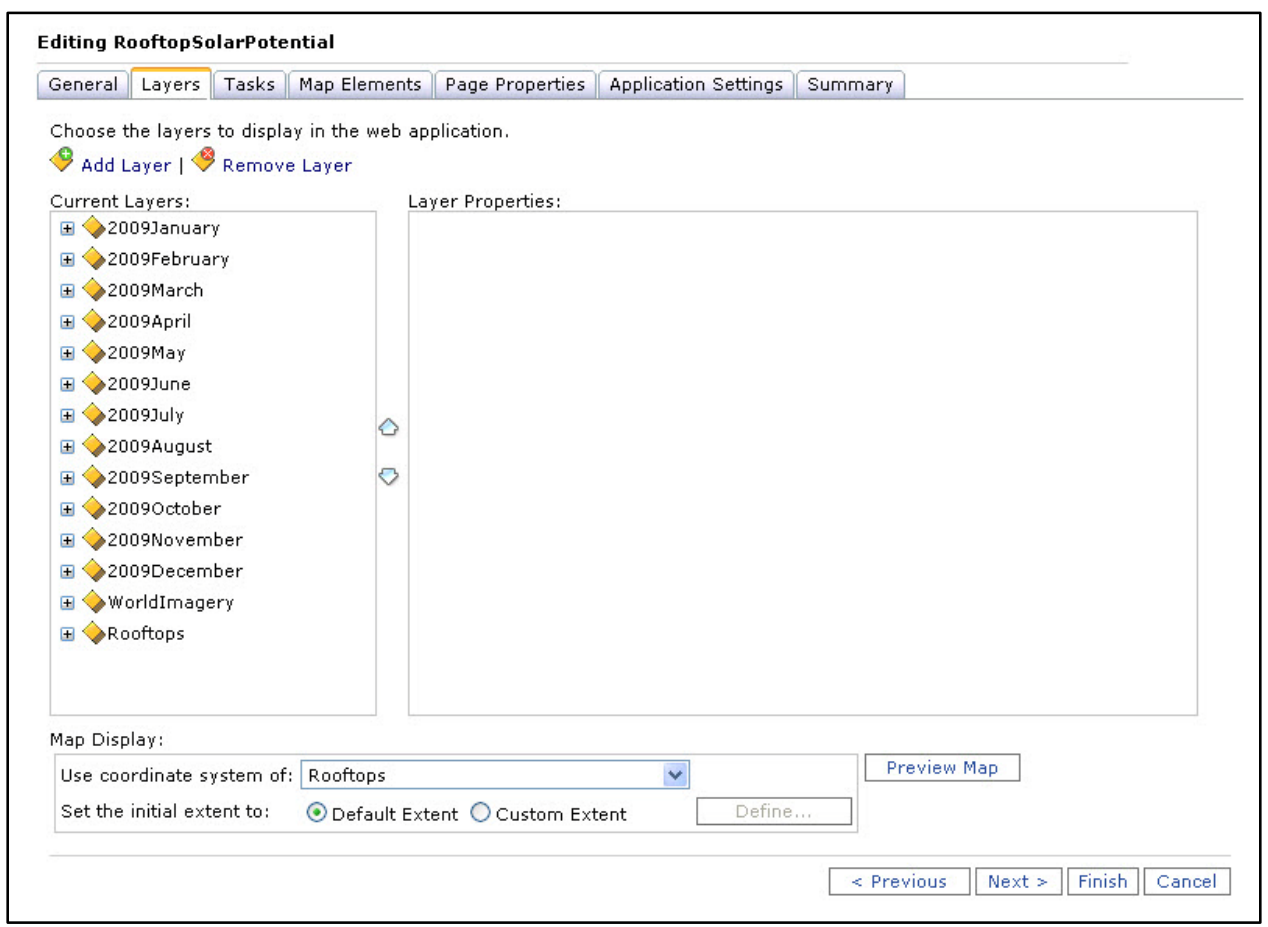

\section{Figure 5-17: Selecting Map Services in ArcGIS Server Manager}

Similarly, the geoprocessing service was added to the Web application through the Supporting Services window under the Tasks tab. Once the geoprocessing service was added, a desired model was selected from a list that appeared by clicking the Configure button (Figure 5-18). 


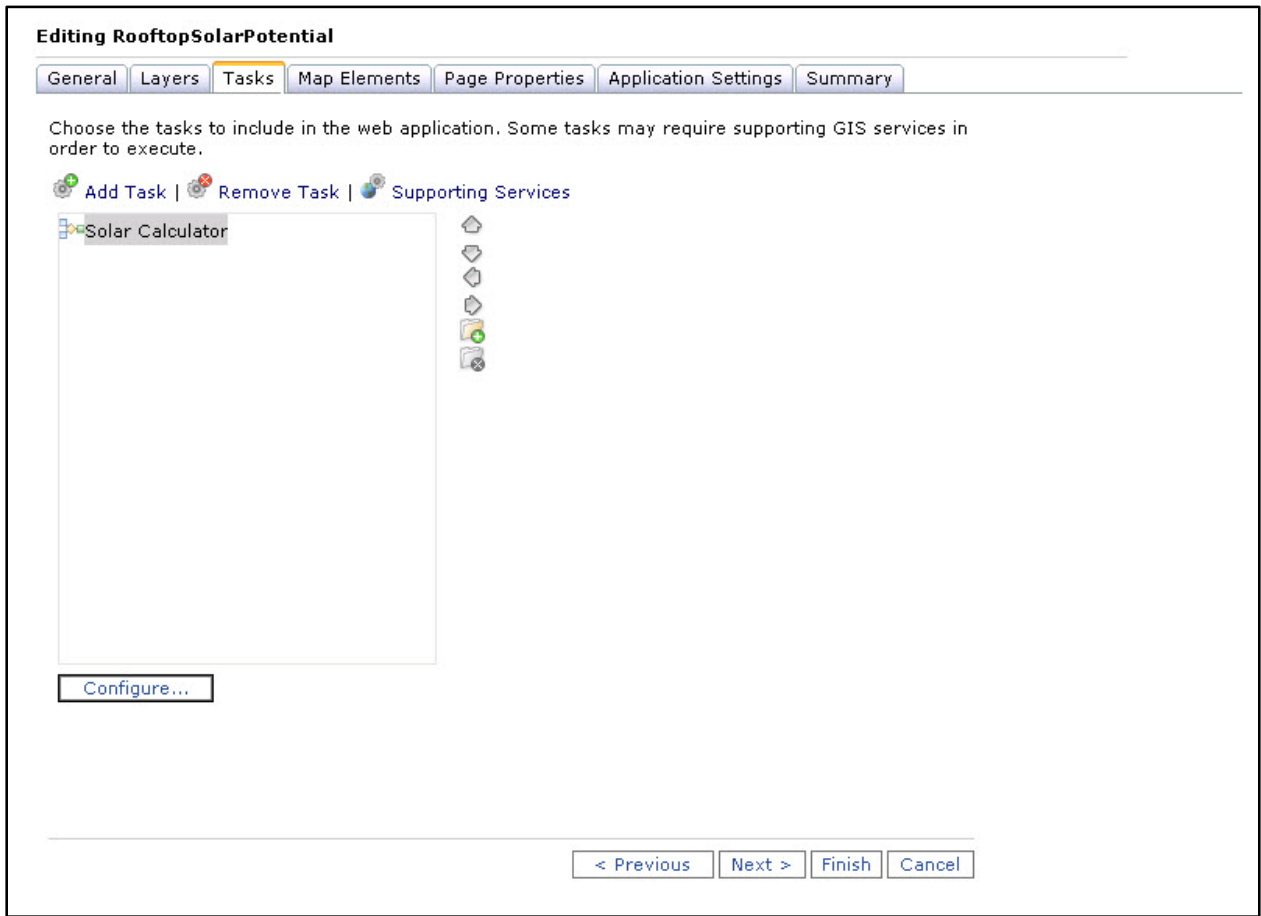

\section{Figure 5-18: Selecting a Geoprocessing Service in ArcGIS Server Manager}

\subsubsection{Developing a Web Application Using JavaScript API}

In addition to the ArcGIS Server Web ADF development framework, ArcGIS API for JavaScript (JavaScript API) was used to embed the map services and tasks in the Web application. In the context of this project, three desired map services were included in the online solar map. First, a world imagery map was added from ArcGIS Online. In order to display the entire study area as the default spatial extent, the default extent of the world imagery map was changed to -13043662.1835921, 4036551.47831127, 13041672.4478528, 4038032.96633455, while its spatial reference ID was set to 102100 (See Appendix A for the complete code). The new default extent of the world imagery map corresponds to the spatial extent of the 2009GlobalSolarRadiation map service in the ArcGIS Services Directory.

Second, the previously published Sample Locations and Rooftop Outlines map layers were added from the 2009GlobalSolarRadiation map service in the ArcGIS Services Directory. In order to retrieve information from the Sample Locations and Rooftop Outlines map layers, the layer IDs were coded as “ 0 ” and “ 1 ” respectively. Similarly, twelve raster datasets map layers that illustrate monthly solar potential values were added from the 2009MonthlySolarPotentials map service in the directory. A series of radio buttons were used in conjunction with the updateLayerVisibility function to control map layers' visibility. By toggling on and off the map layers, users can better understand the seasonal patterns of solar potentials throughout the year 2009. At the same time, a graduated symbol of each map layer was added to the right side of the map. 
In addition to map display, the online solar map has the Identify function. Unlike the Query function, which can "work with [only] one layer at a time," the Identify function can "retrieve information from multiple layers at [a time]" (ESRI, 2010h). Once a target rooftop is identified on the map, attribute data are retrieved from each map layer and displayed in an information window with the tabular format. The monthly solar potential values in $\mathrm{kWh}$ are retrieved from the JAN, FEB, MAR, APR, MAR, JUN, JUL, AUG, SEP, OCT, NOV, and DEC fields of the Sample Locations layer, while the building name and its rooftop area $\left(\mathrm{m}^{2}\right)$ are extracted from the Name and Shape_Area fields of the Rooftop Outlines layer. The monthly solar potential values are displayed in both table and chart form using functions such as layerTabContent and addToMap. In addition, depending on a map scale, multiple sample points may be selected from the Sample Locations layer, in which case the Identify task selects sample points within a distance/tolerance of 3 screen pixels from the specified point and then calculates mean solar potential values by month for those selected sample points. A new tab of "Definition" was added in order to describe how the solar potential values for an area of interest were calculated.

\subsection{Summary}

The Solar Radiation Analysis model consists of a series of geoprocessing tools. First, LiDAR point clouds were converted to a DSM and sample locations point features. These were used as primary inputs of the Points Solar Radiation tool to calculate solar potential values for the sample locations, which were determined by the point features. Once the solar potential values were calculated, solar potential values in areas between the sample locations were estimated by using the IDW interpolation method. Based on the estimated values, continuous surface maps of monthly solar potential values were created for entire rooftops. The ArcGIS Server Web ADF application was used first to display the solar potential maps and calculate the solar potential values for a given location on-the-fly. Based on lessons learned from this first attempt, a Web application using JavaScript API was also created. Not only were the monthly solar potential values displayed, but the average solar potential values for the selected sample locations were calculated and displayed in both table and chart form. The next chapter will examine the results of the Solar Radiation Analysis model and several attempts to develop Web applications. 



\section{Chapter 6 - Results and Analysis}

Chapter 6 presents major project results and discusses the issues encountered during the implementation. First, the advantages of using high resolution LiDAR data in solar potential calculation are discussed. Second, local and seasonal patterns of solar potential values calculated at sample points are examined. Third, characteristics of the IDW interpolation technique that was used to create continuous solar potential maps are discussed. Lastly, two Web applications implemented with different techniques (ArcGIS Server Web ADF and JavaScript API) are examined and key functions are illustrated.

\subsection{Primary Inputs Generated from LiDAR Point Clouds}

High resolution LiDAR data were used to generate a DSM and sample locations point features. Figure 6-1 shows a 2m DSM generated from the LiDAR point clouds (Top) and a USGS 30m DEM (Bottom) for the University of Redlands campus. In the 2m DSM, surface areas with relatively higher elevations are shown in lighter grey, while surface areas with relatively lower elevations are indicated in darker grey. The USGS 30m DEM does not clearly show varying elevation data within the same spatial extent. The DSM generated from the high resolution LiDAR data can reveal more detailed surface features on building rooftops. 

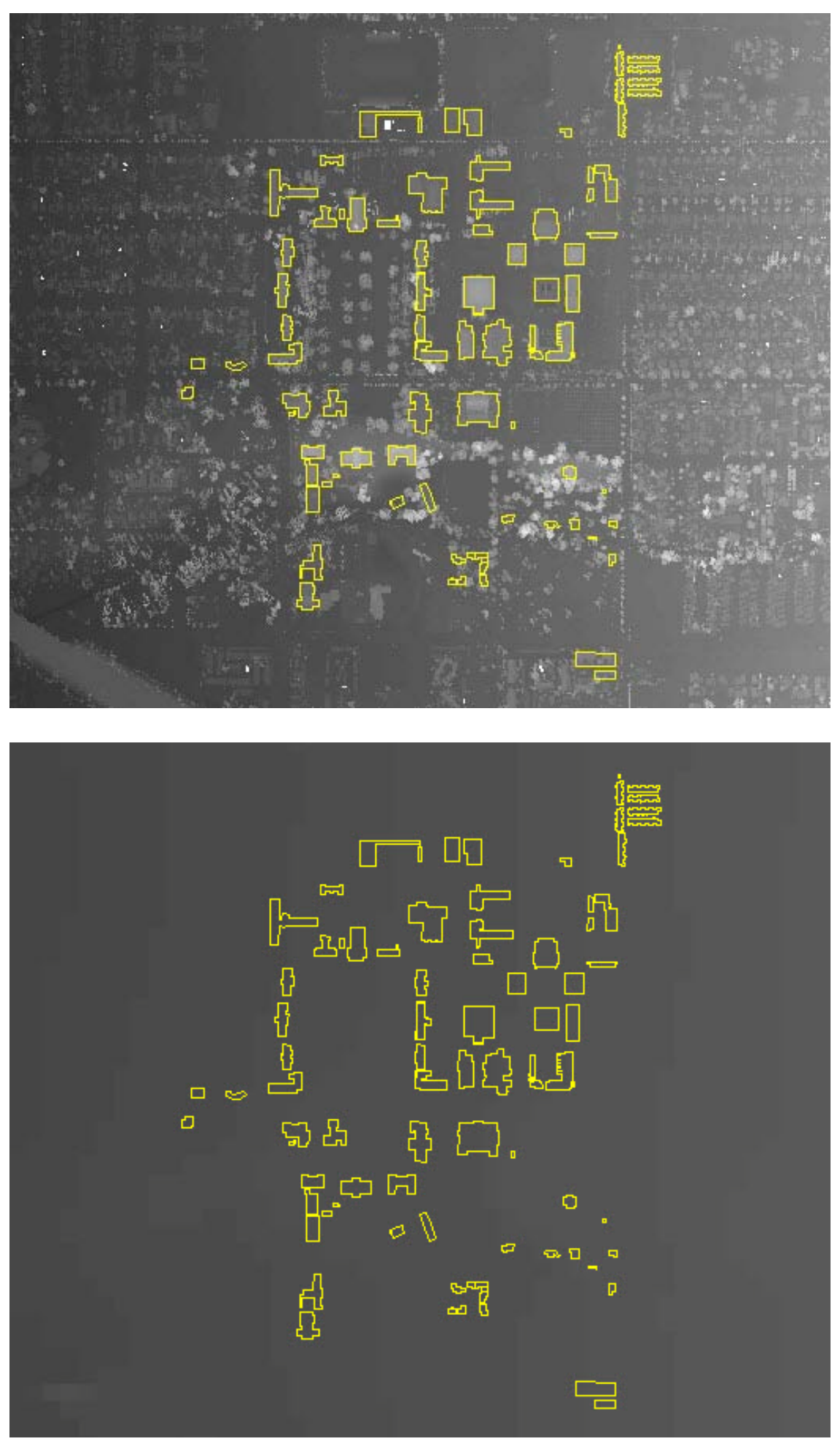

Figure 6-1: 2m DSM (Top) and 30m DEM (Bottom) for the Study Area

Figure 6-2 shows sample locations point features generated from the same LiDAR point clouds. These point features were LiDAR point clouds that were classified as 
buildings and located within the rooftop outline polygons. These point features were used as sample locations for calculating solar potentials on the rooftop surfaces.

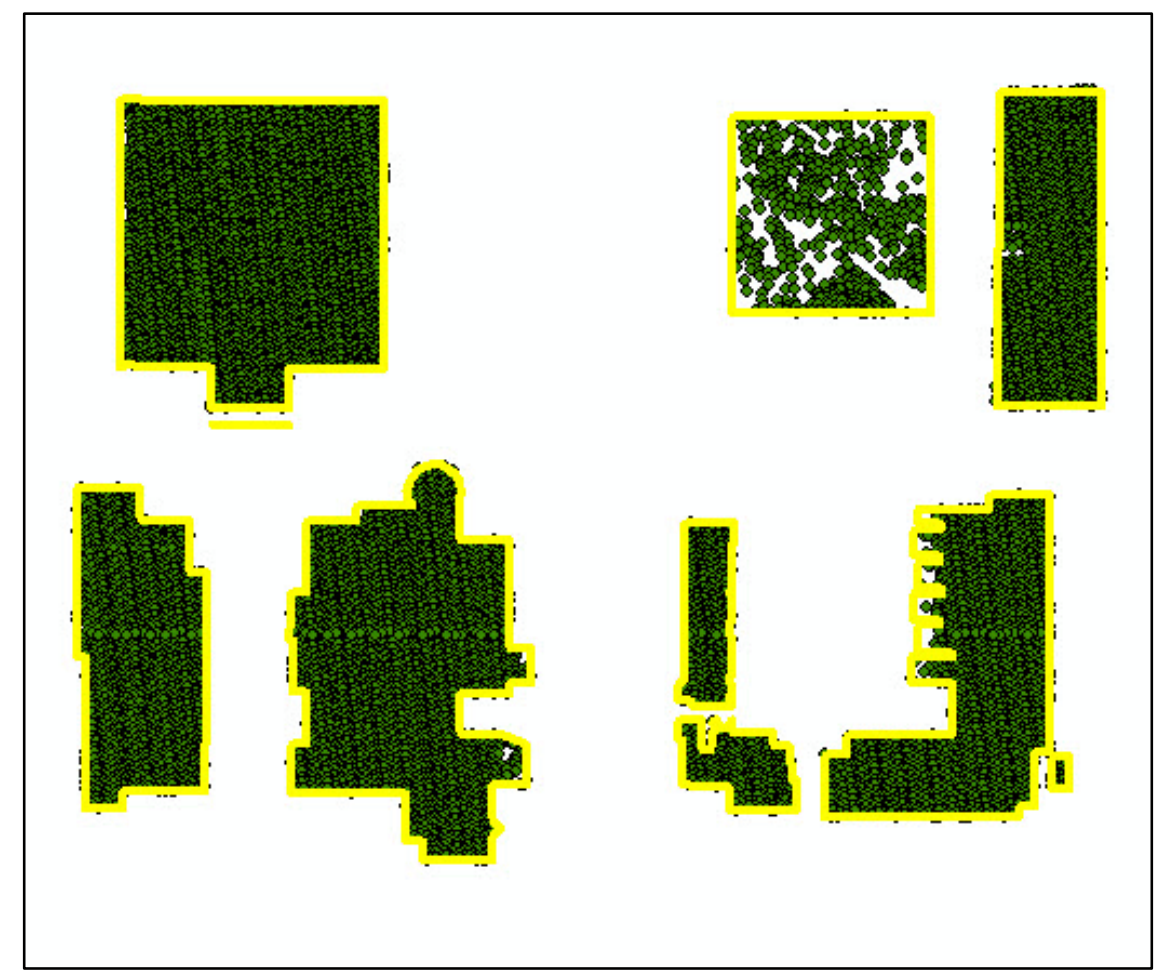

Figure 6-2: Sample Points on Building Rooftop Surfaces

\subsection{Points Solar Radiation Analysis}

The amount of global radiation was calculated at a total of 73,158 locations on 114 building rooftops. The analysis results revealed local and seasonal patterns of the global radiation in this study area. Two sample points on Armacost Library, one located on the south side and the other on the north side of the rooftop, were randomly selected and differences in solar potential values were examined (Figure 6-3). Solar potential values in January at the south and north side sample locations were estimated at $68.0 \mathrm{kWh}$ and $37.3 \mathrm{kWh}$, while the solar potential values in July at the same sample locations were estimated at $186.7 \mathrm{kWh}$ and $179.2 \mathrm{kWh}$ respectively. In other words, the July solar potential values were twice or more higher than the January solar potential values. This represents a typical seasonal pattern of global solar radiation in this study area. 


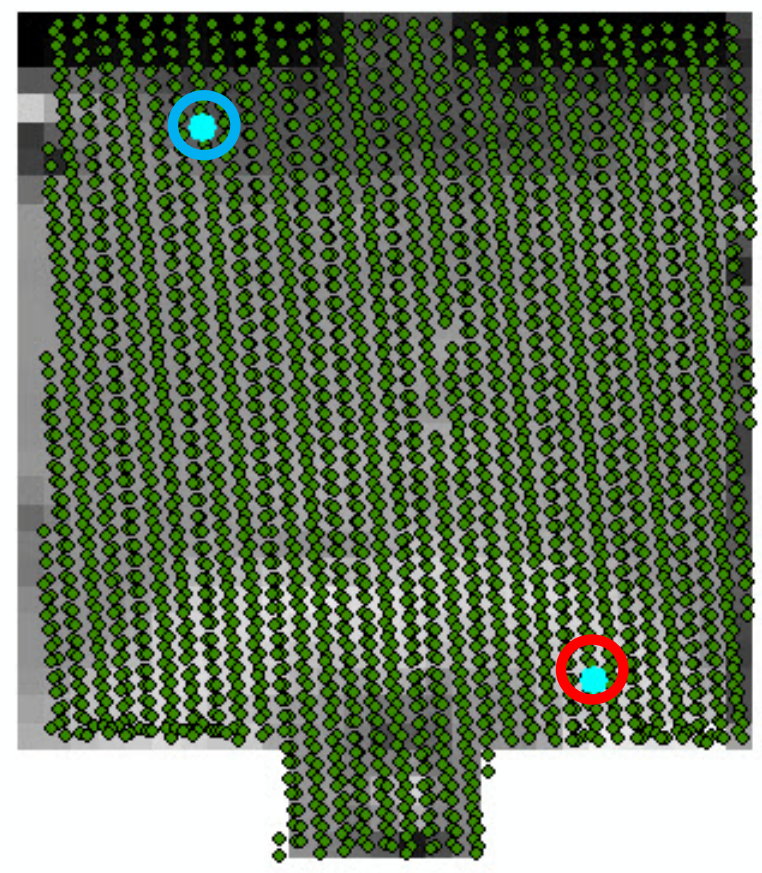

\begin{tabular}{|c|c|c|c|c|c|c|c|c|c|c|}
\hline \multicolumn{9}{|c|}{ 囲 Selected Attributes of Solar Potential } & \multicolumn{2}{|c|}{\begin{tabular}{|l|l|l|}
$口$ & 回 & $\mathbf{X}$ \\
\end{tabular}} \\
\hline & OBJECTID * & Shape * & & JAN & FEB & MAR & APR & MAY & JUN & JUL \\
\hline & 35517 & Point & & 68 & 84.6 & 131.4 & 158.8 & 182.8 & 182.7 & 186.7 \\
\hline & 40798 & Point & & 37.3 & 53.6 & 97.5 & 135.4 & 171.4 & 176.5 & 179.2 \\
\hline \multirow[t]{2}{*}{4} & (2) & III & & $\square$ & & & \multirow{2}{*}{\multicolumn{3}{|c|}{ Records ( 2 out of 73158 Selected) }} & 1 \\
\hline & Record: id & 4 & ' & $|n|$ & Show: All & Selected & & & & Options \\
\hline
\end{tabular}

Figure 6-3: Local Solar Radiation Pattern on South (Red, ID: 35517) and North (Blue, ID: 40798) Facing Rooftops

At the same time, Figure 6-4 shows that monthly solar potential values on the south facing rooftop are usually higher than solar potential values on the north facing rooftop throughout the year. This confirms that surface areas on south-facing rooftops receive a greater amount of global solar radiation than surface areas on north-facing rooftops. However, the difference significantly decreases in the summer when compared to the difference in the winter. This is likely due to the angle of the sun. 


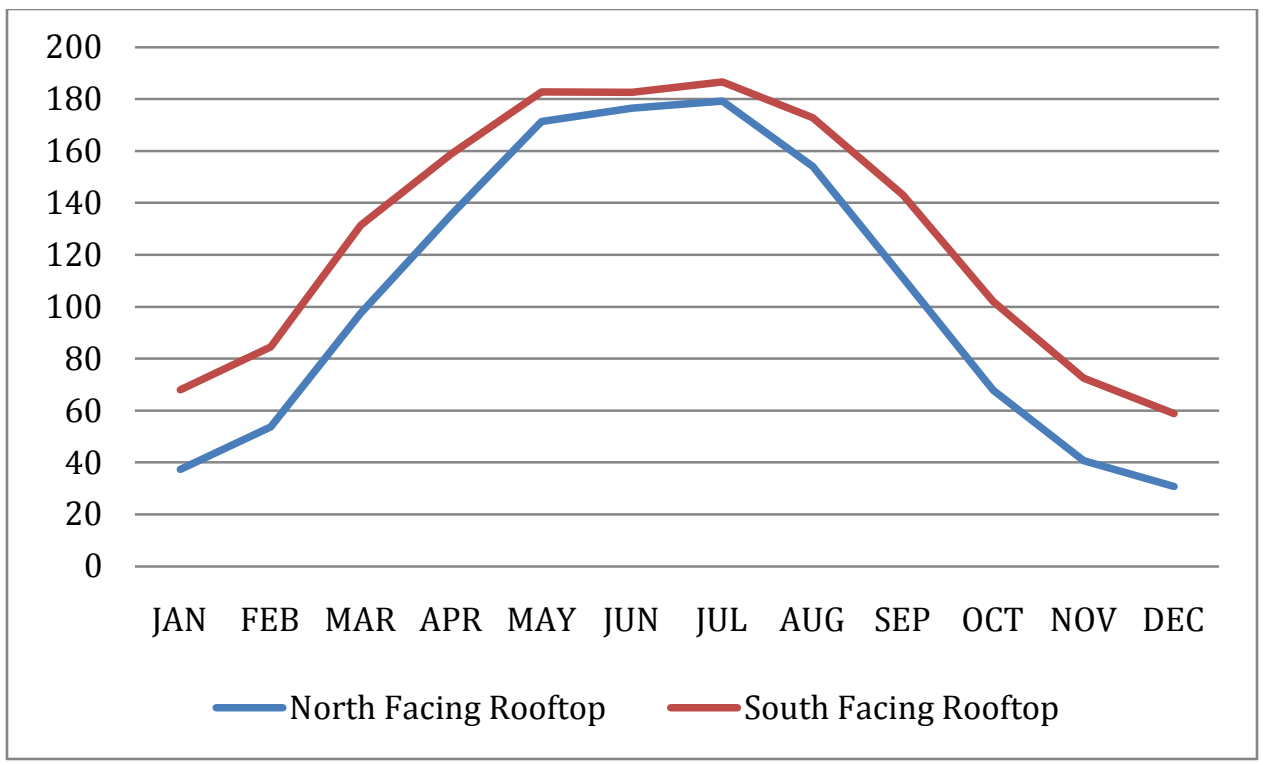

Figure 6-4: Seasonal Solar Radiation Pattern on South (Red) and North (Blue) Facing Rooftops

\subsection{IDW Interpolation Result}

The IDW interpolation technique was used to interpolate solar potential values across rooftop surfaces. It assigns weights based on the distances between the interpolation point and sample points. The influence from samples becomes more significant as the interpolation point is closer to the samples (ESRI, 2007). Only a subset of sample points within the specific search radius $(3 \mathrm{~m})$ was used to include the neighboring sample points on the same building. Figure 6-5 shows continuous surfaces of solar potential values on building rooftops for January 2009. 


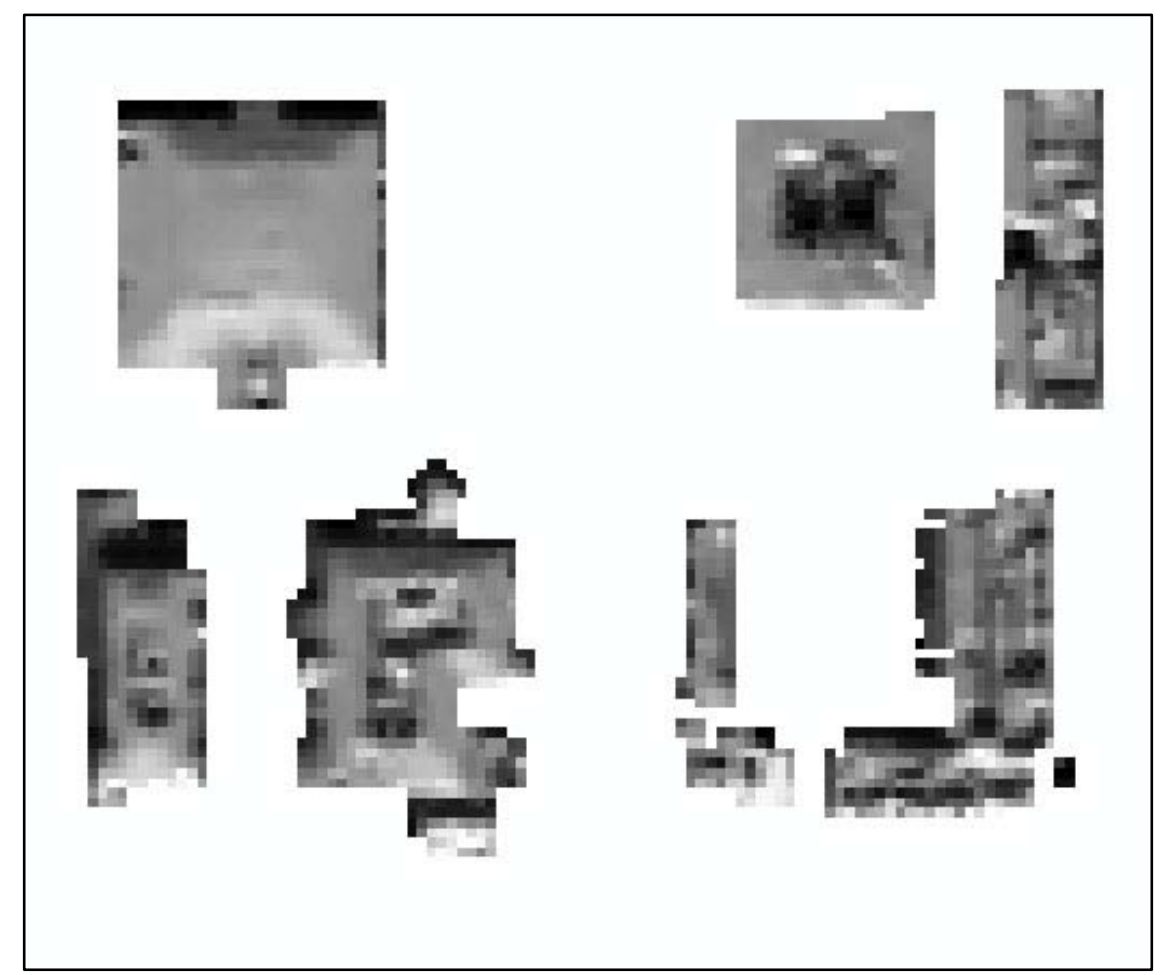

Figure 6-5: Continuous Surface Map of Solar Potential for January 2009

\subsection{ArcGIS Server Web ADF Mapping Applications}

When implementing the online solar map, the first attempt was to use ArcGIS Server Web ADF. The online mapping application developed with ArcGIS Server Web ADF provides users with direct access to the local and seasonal patterns of incoming solar radiation across each building rooftop (Figure 6-6). This application involved twelve map layers ranging from monthly solar potential values for January 2009 (0901solarpotential) through December 2009 (0912solarpotential). The main area of the user interface displays interpolated surface maps of the monthly solar potential values on an imagery base map obtained from ArcGIS Online. To view desired solar potential information for building rooftops, users can zoom in and out of the map, pan the map, and change the map extent. Users can also define the $\mathrm{X}$ and $\mathrm{Y}$ coordinates of a location, measure a distance between two locations, calculate an area of a polygon, and show/hide an overview map. Further, users can toggle map layers’ visibilities and view graduated symbols of the map layers in the Map Contents section. 


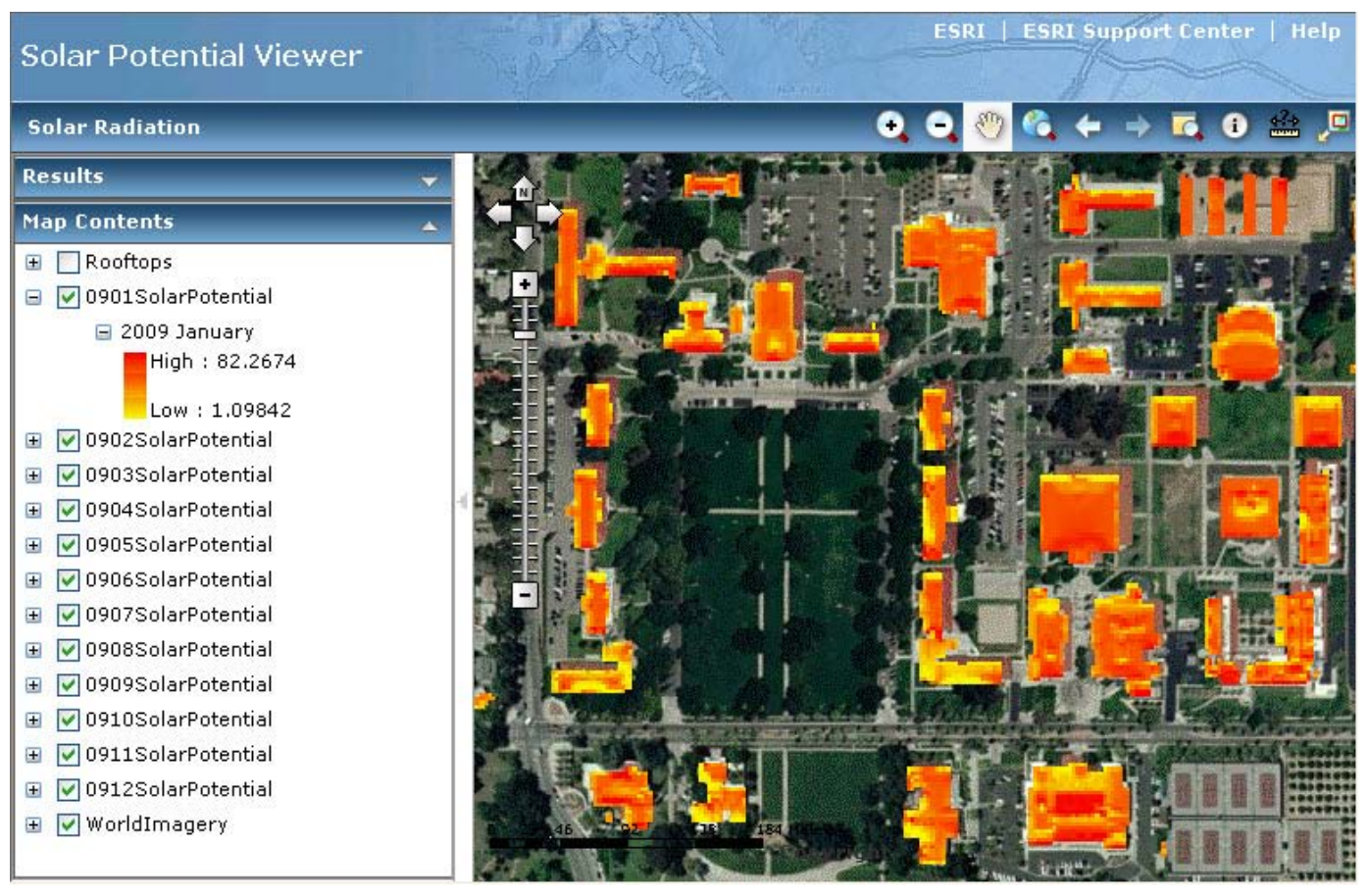

\section{Figure 6-6: Display Monthly Solar Potential on Building Rooftops}

Besides the display function, this online mapping application also provides users with a tool to calculate the amount of global radiation at a location of interest on-the-fly. One geoprocessing service in the ArcGIS Server was added to this application. Users can draw a polygon on a building rooftop using the Add New Feature tool in the Solar Radiation window (Figure 6-7). The geoprocessing service first converts the polygon feature to its centroid point and then uses both the point and the DSM as primary inputs of the Points Solar Radiation tool. Once the solar radiation analysis is completed, monthly solar potential values at a location of the converted point are displayed in the Results section. 


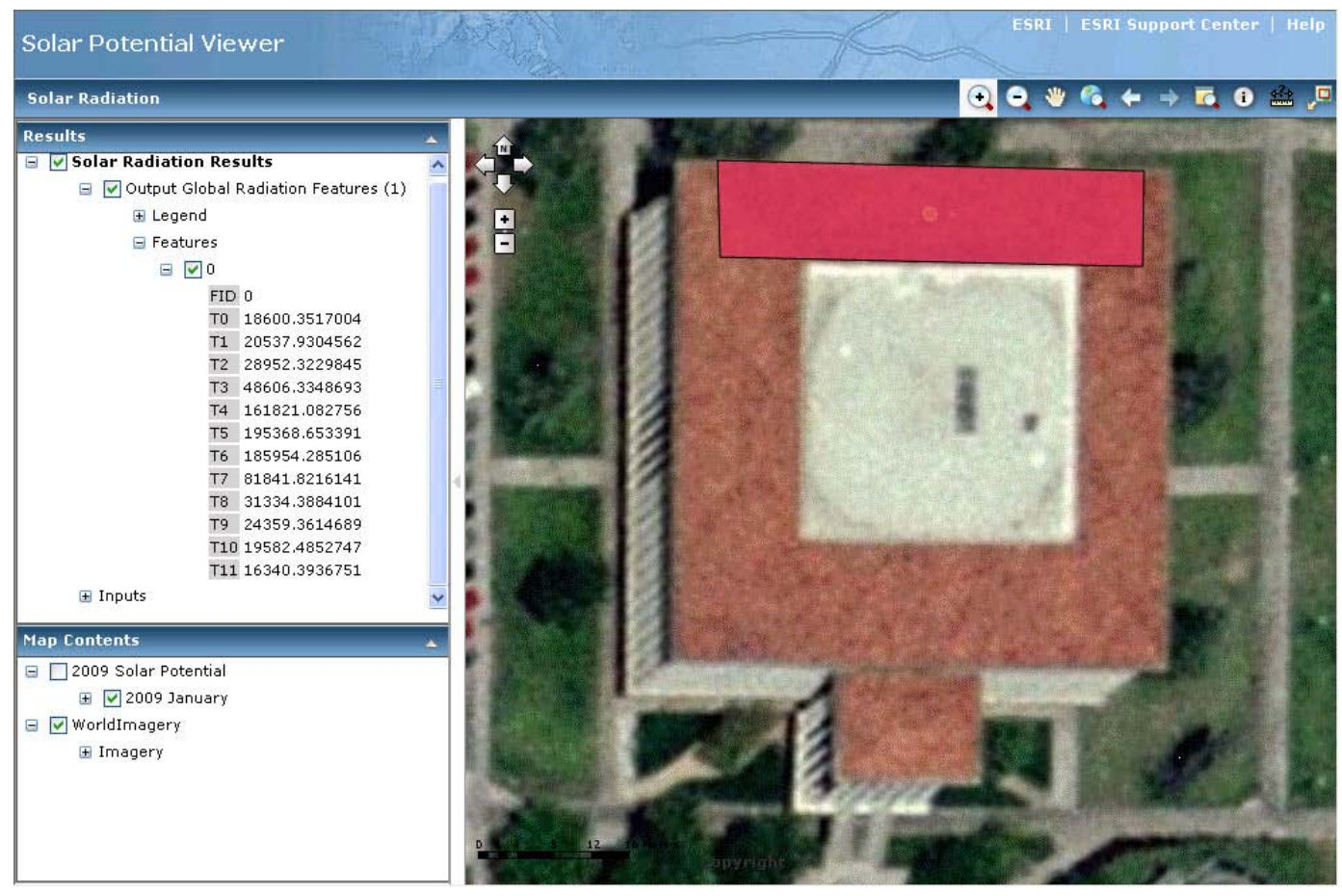

Figure 6-7: Calculate Solar Potential for Given Location On-the-Fly

Although this application includes the on-the-fly solar energy calculation, this geoprocessing service only accepts one sample point at a time. In addition, although it is technically attainable, presenting the final solar energy variations across different months in chart form was not successfully implemented in this attempt. Therefore, a second attempt was made using JavaScript API. This application was created to select multiple sample points and then calculate the mean solar potential values of the selected sample points. Further, this application was also developed to display the monthly mean solar potential values in both chart and table form.

\subsection{ArcGIS API for JavaScript Mapping Applications}

The Web mapping application using JavaScript API, illustrated in Figure 6-8, provides users with tools that can calculate the average solar potential values of neighboring sample points from a given location. 


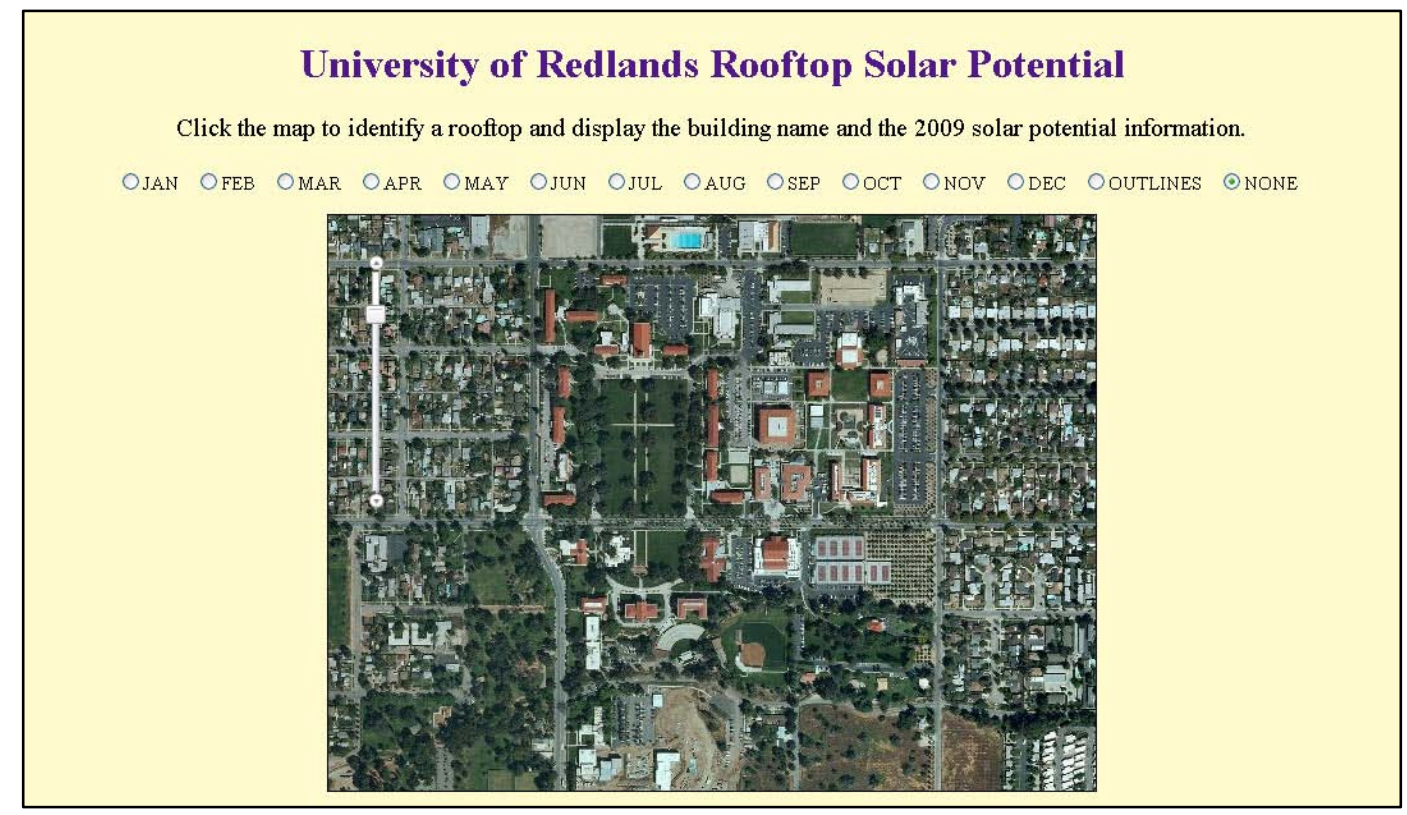

Figure 6-8: University of Redlands Rooftop Solar Potential Viewer Interface

First, this online solar map enables users to display monthly solar potential maps by clicking a radio button placed in the middle of the interface to show/hide a desired map or rooftop outline. An associated graduated symbol also appears on the right side of the interface to inform users how to read the map (Figure 6-9). Further, because the solar potential map layers are partially transparent, it is easier for users to view desired building features and solar potential information at the same time.

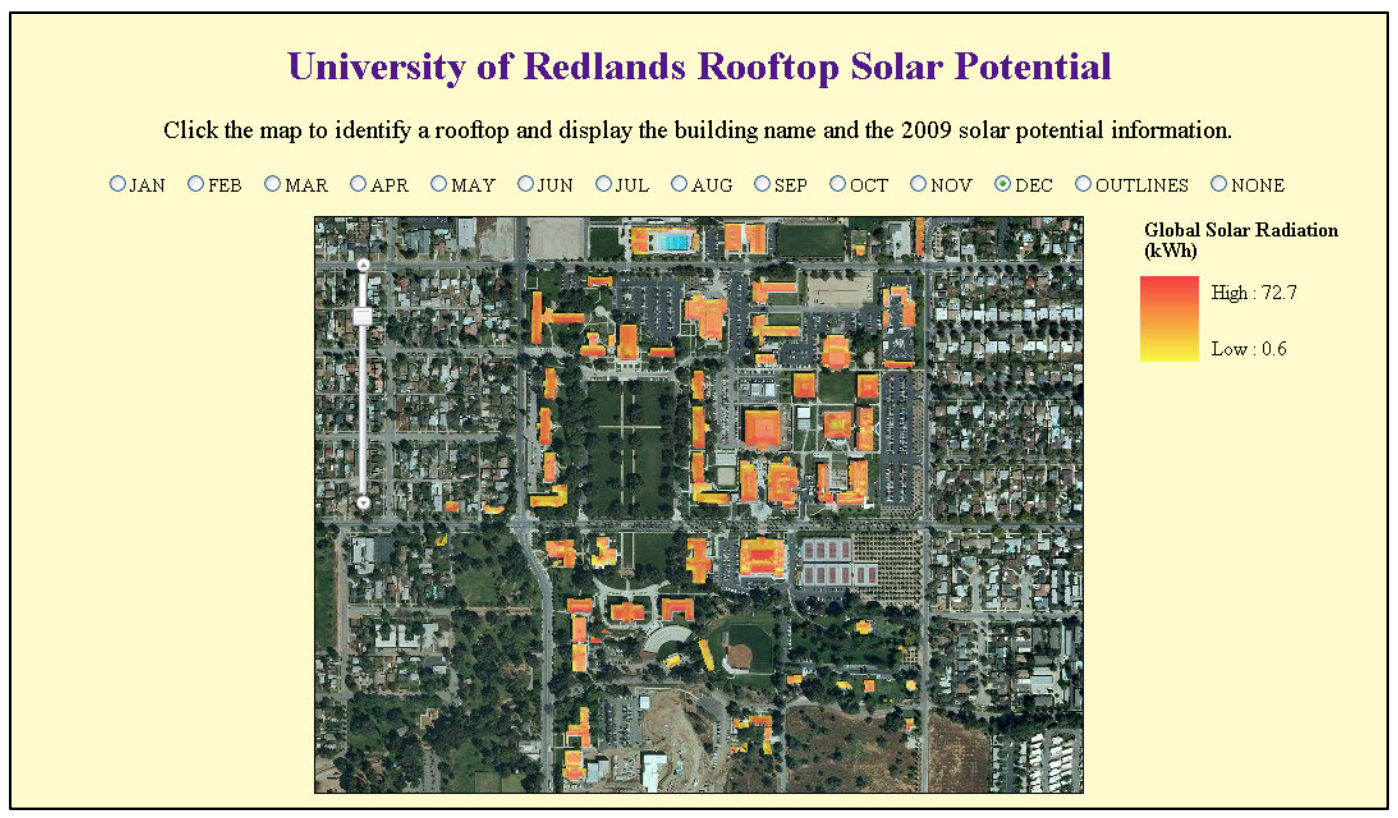

Figure 6-9: Graduated Symbol for Monthly Solar Potential of December 2009 
Second, this solar map allows users to click a map to calculate the solar potential at that particular location based on the neighboring sample locations. Clicking outside building boundaries returns no result. The search radius for the neighboring sample points is 3 screen pixels, and the returned value for the specified location is the average solar potential of all the neighboring sample locations. This output, the number of selected sample points, as well as a building name and its area, are summarized in a tabular format (Figure 6-10, 6-11, 6-12).

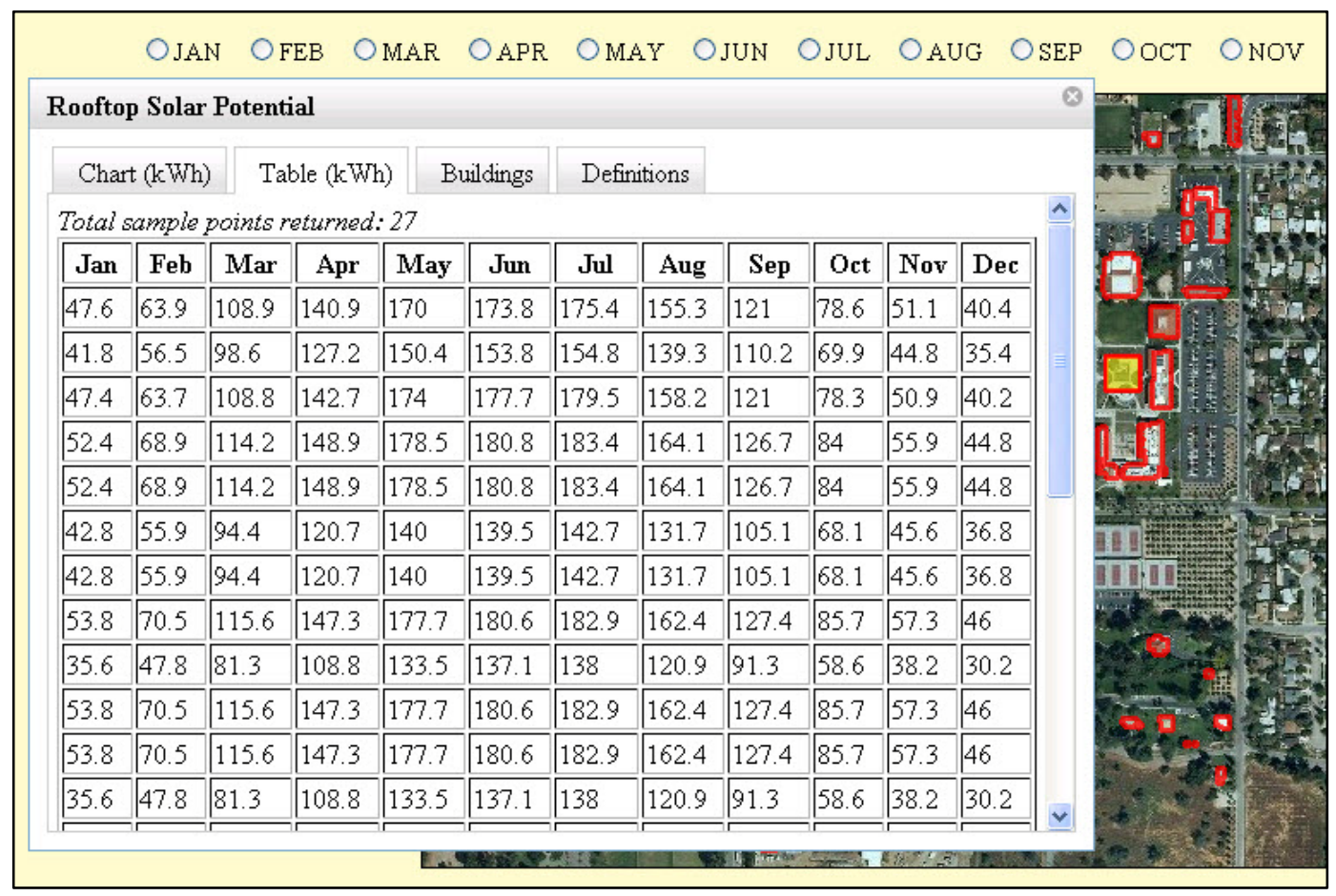

Figure 6-10: Solar Potential for Each Selected Samples in Table Tab 


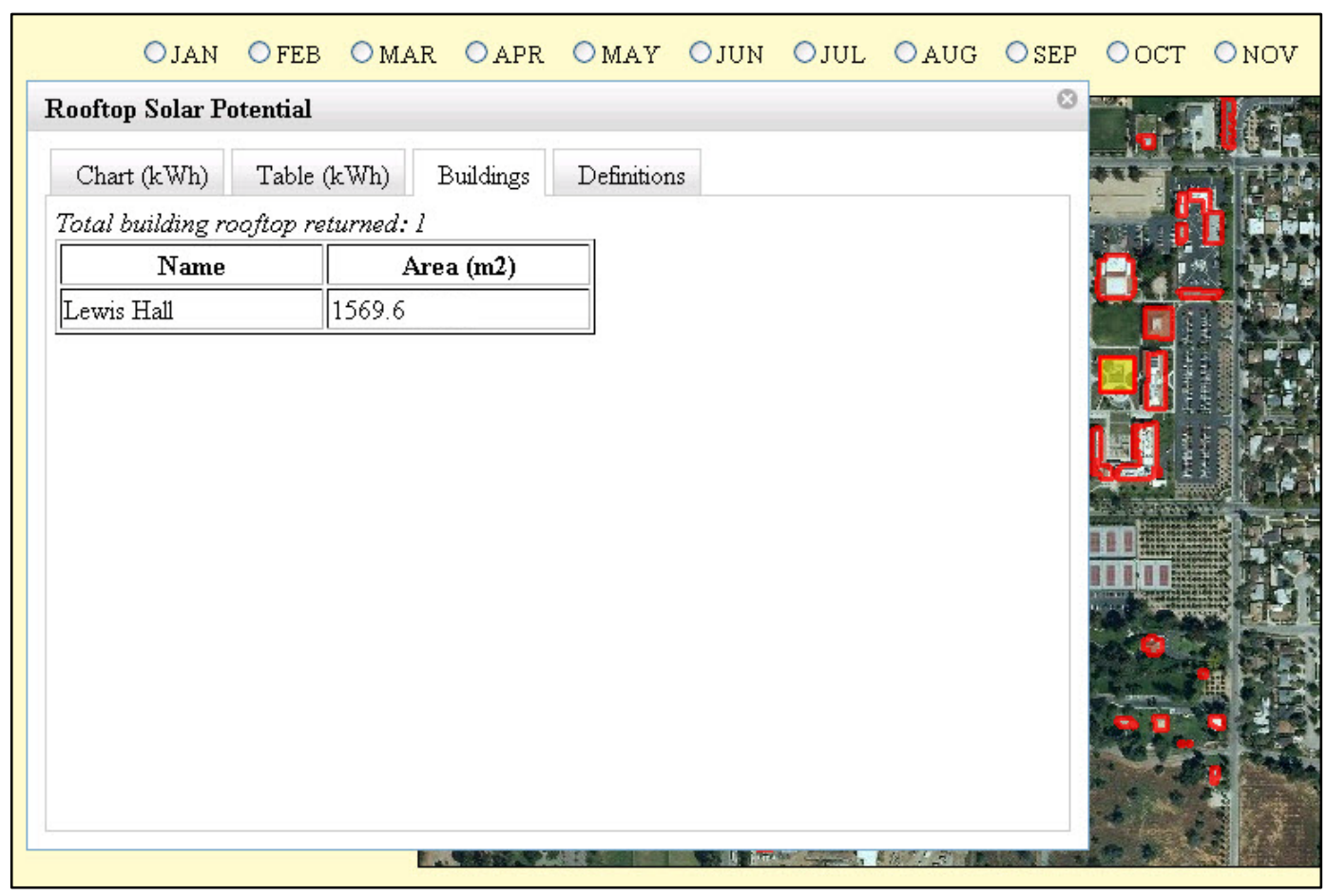

Figure 6-11: Building Attribute Data in Buildings Tab

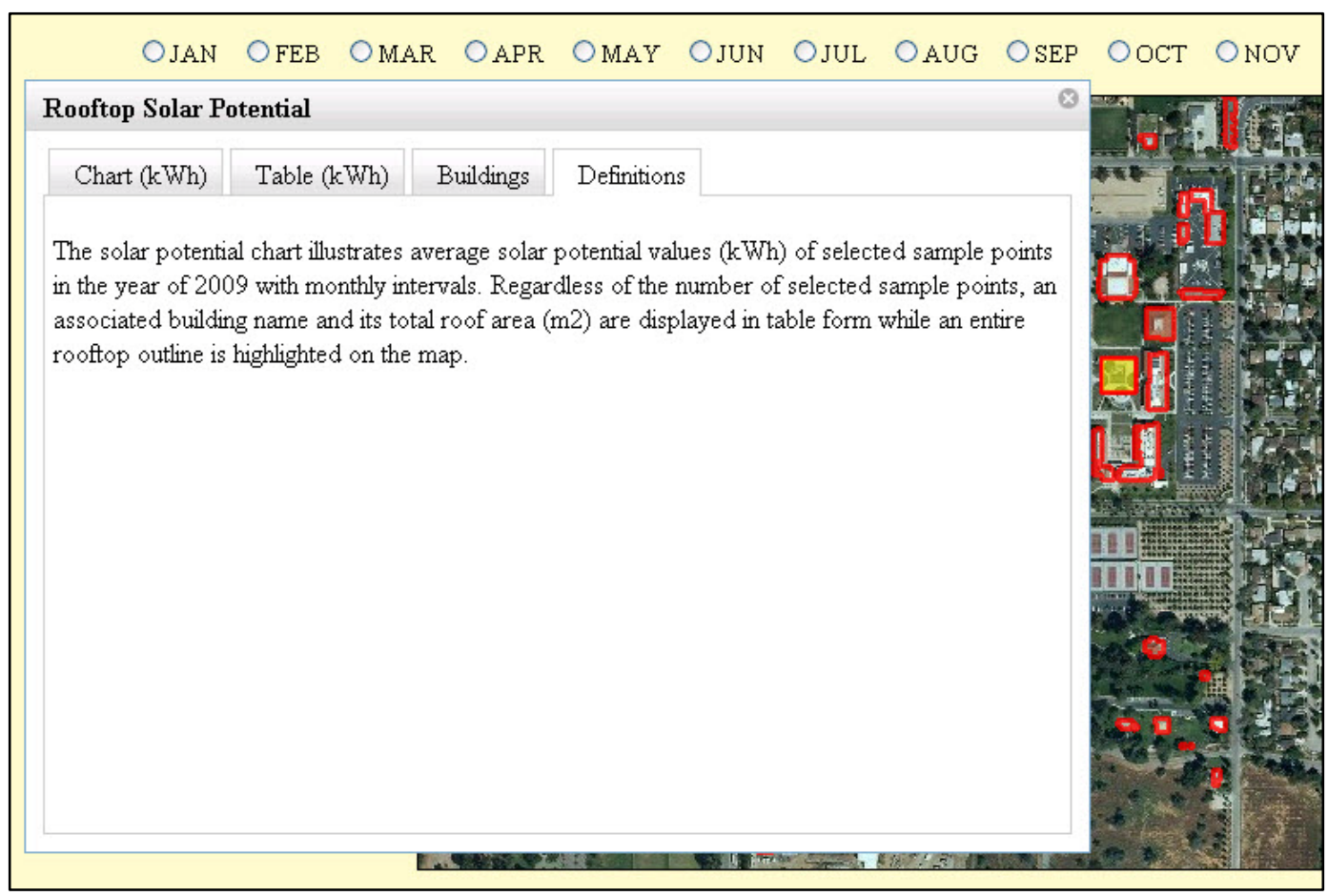

Figure 6-12: Definition of Average Solar Potential Calculation in Definition Tab 
Monthly solar potential values of the selected location are then calculated and displayed in chart form while highlighting the entire rooftop outlines (Figure 6-13). A combination of these functions allows users not only to better understand local patterns of global radiation and identify ideal locations in terms of the global radiation, but also to view the seasonal patterns of the global radiation.

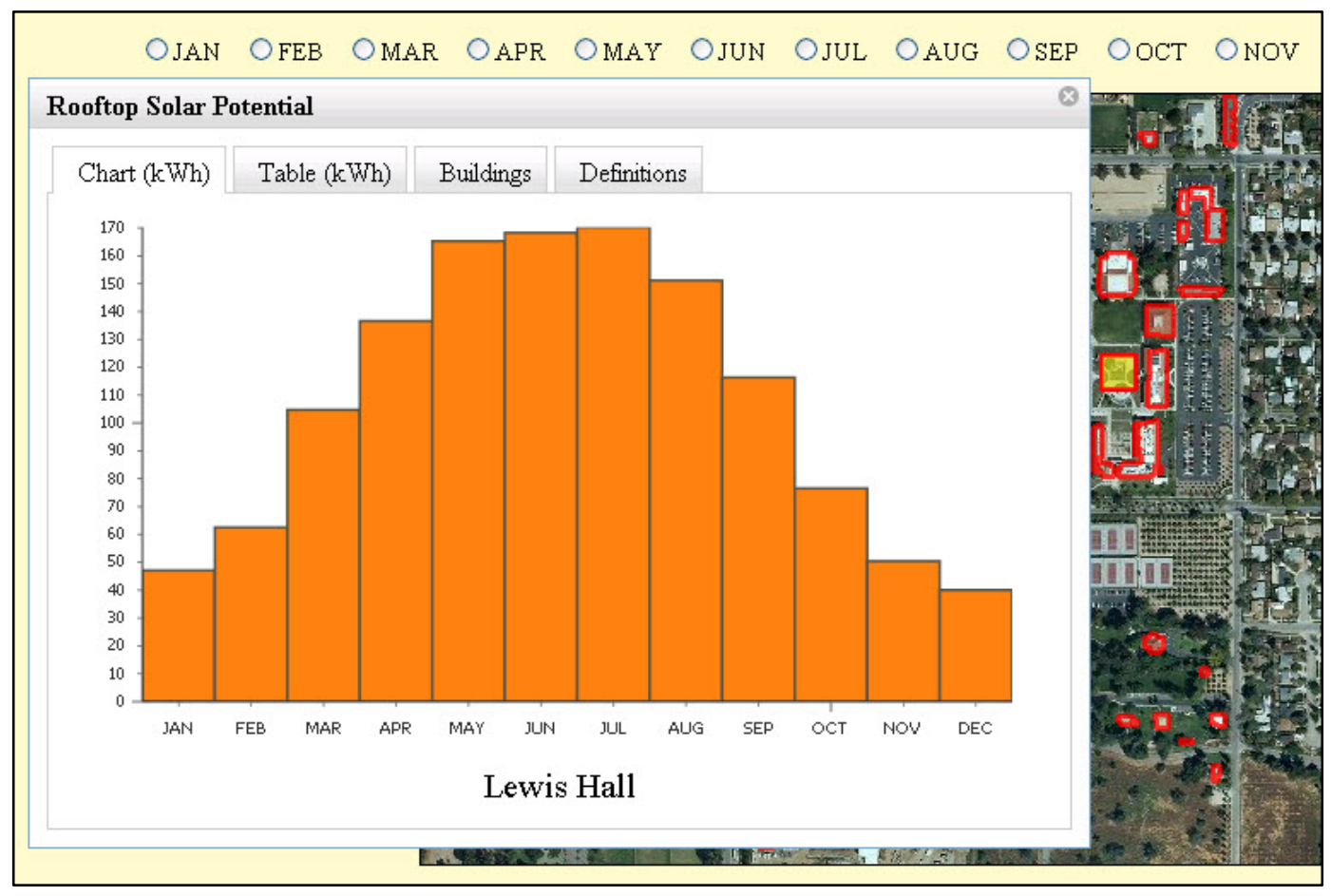

Figure 6-13: Mean Solar Potential of Selected Samples in Chart Tab

\subsection{Summary}

The DSM and sample locations point feature class that were generated from high resolution LiDAR data were used to detect surface features on building rooftops and to calculate solar potential values at sample locations on building rooftops. Using the IDW interpolation technique, continuous surfaces were further created by interpolating the solar potential values that were calculated at the sample locations. These results reveal local and seasonal patterns of solar potential values on the building rooftops. At the same time, Web mapping applications provide an effective means to distribute the information about solar potentials. The previously discussed ArcGIS ADF and JavaScript API mapping applications both assist in not only displaying local and seasonal patterns of solar potentials across building rooftops, but also in processing the calculated solar potential values to obtain and distribute desired information in the desired formats. These functions potentially enable users to identify ideal locations for solar panels for maximizing the amount of solar energy while maintaining building aesthetics. Due to the initial requirement of having a chart output, the online solar map developed with JavaScript API was included in the final deliverables. 


\section{Chapter 7 - Conclusions and Future Work}

In order to assist in the decision-making process of the City of Redlands regarding the installation of solar panels, this project developed a Web-based solar map using DEMs generated from high resolution LiDAR data. The University of Redlands campus was selected as the study area. This online solar map provides the means to inform users of the latest updates regarding solar radiation, and also to support their decision-making process by identifying ideal locations for solar panels for maximizing their benefits.

High resolution LiDAR data were successfully used to understand local and seasonal patterns of solar potential on building rooftops. The LiDAR point clouds were converted to a DSM with a smaller cell size to reveal detailed surface features on building rooftops. The LiDAR point clouds were also converted to point features to use as sample locations for solar potential calculation on building rooftops. Using the Points Solar Radiation tool in the ArcGIS Spatial Analyst toolbox, seasonal patterns of solar potential on building rooftops were clearly revealed. More significantly, considering unique surface features of each building rooftop, local patterns of solar potential were also identified by calculating solar potential values at various sample locations and estimating the areas between the sample locations using an interpolation technique.

The online solar map was developed as an interactive tool that can analyze and display the latest updates of information about solar energy. The ArcGIS Server Web ADF development framework was used first to display monthly solar potential values for the year 2009. Solar potential values for a central point of a given polygon were also calculated on-the-fly. Based on lessons learned from the first attempt with the ArcGIS Server Web ADF, not only were the monthly solar potential values displayed, but the average solar potential values of selected sample points were also calculated and displayed in both chart and table form. Although the initially proposed solution to develop a Web application using API for Flex was shifted to JavaScript API as the project evolved, the several attempts to develop a Web application with two different development frameworks met the functional requirements about Web mapping applications.

\subsection{Future Work}

Using the same primary inputs can make the Solar Radiation Analysis model and the Web application meet different needs. The original plan for this project was to calculate the solar potential for building rooftops for the entire City of Redlands. Due to time constraints, the project scope was scaled down to the university campus. However, because the city owns the same types of primary inputs, including LiDAR data and building footprint data, the model and the Web application for this study area can be applied to the entire city. At the same time, because the LiDAR data contains all points that have been classified by a private vendor into different class codes depending on surface features, the client can calculate the amount of solar potential not only on building rooftops but also on the surface of roads by changing desired class codes in the 
LAS to Multipoint tool and possibly adding new tools to the model. This will help the city develop models to further analyze solar radiation in regards to "green islands" and the effect that trees and other vegetation have on the ambient temperature.

Converting high resolution LiDAR data and calculating solar potential for a number of building rooftops generally require a large amount of time. In the context of this project, the Solar Radiation Analysis model required approximately 25 minutes to complete a sequence of algorithms. Because this study area is much smaller than the entire City of Redlands, converting LiDAR point clouds to a DSM with a cell size of 2 meters and a singlepart point feature class and calculating solar potential for a number of building rooftops in the city would need much more time. This project used only tools that are available in the ArcGIS Desktop environment, in conjunction with the 3D Analyst and Spatial Analyst extensions. Using a plug-in that has additional capabilities to process LiDAR data can potentially enhance processing efficiency and also improve analysis results. At the same time, this project used relatively high numbers for key parameters, such as skysize/resolution and calculation directions in the Points Solar Radiation tool. These parameters can considerably increase the amount of processing time. In order to calculate solar potential for the entire city, therefore, the model should be examined further and even modified to accept appropriate parameters that can minimize the amount of processing time while retaining desired accuracy.

Using the Area Solar Radiation tool could be another approach to address solar energy mapping. The Area Solar Radiation tool, which is located in the Spatial Analyst toolbox, can calculate the incoming solar radiation across each building rooftop by repeating the calculations for each location in the input DSM (ESRI, 2010a). The Points Solar Radiation tool was implemented in this project because this tool allows users to select a point of interest by clicking on the map, and then calculate solar potential values for the given location on-the-fly. It is possible that the Area Solar Radiation tool would replace the Points Solar Radiation tool in the model to address different demands and desires.

New functions could be added to the current Web application in the future. This project focused on a Web application that involves three functions, including displaying monthly solar potential maps, identifying and calculating the average solar potential values of selected features on-the-fly, and displaying the analysis result in both chart and table form. Like other mapping examples discussed in Chapter 2, future additions could include displaying a variety of information, such as locations of installation sites, the estimated daily solar potential, and the annual cost and carbon dioxide savings of each building rooftop. At the same time, because the online solar map for the entire city will cover a much larger area and involve many more building polygons than this project, the geocoding tool could be another future addition to identify a target rooftop using the address of the building and saving time for searching the rooftop manually. 


\section{Works Cited}

ASPRS. (2010). What is the LAS Format?. Retrieved May 15, 2010 from http://www.asprs.org/society/committees/standards/lidar_exchange_format.html

Bolstad, P. (2008a). An Introduction. GIS Fundamentals: A first text on geographic information systems $-3^{\text {rd }}$ ed. (pp. 1-24). White Bear Lake, MN: Eider Press.

Bolstad, P. (2008b). Aerial and Satellite Images. GIS Fundamentals: A first text on geographic information systems $-3^{\text {rd }}$ ed. (pp. 211-258). White Bear Lake, MN: Eider Press.

Campagna, M. (2006). GIS for Sustainable Development. In M. Campagna (Ed.), GIS for Sustainable Development. (pp. 3-20). Boca Raton, FL: CRC Press.

Chang, K. (2006). Raster Data Model. Introduction to Geographic Information Systems $3^{\text {rd }}$ ed. (pp. 75-95). New York, NY: McGraw-Hill Companies, Inc.

City of Boston. (2009). Solar Boston. Retrieved October 16, 2009 from http://www.cityofboston.gov/climate/solar.asp

DeMeritt, M. (2008, Fall). Boston Showcases Solar Power Potential with Web GIS. ESRI ArcNews Online. Retrieved October 13, 2009 from http://www.esri.com/news/arcnews/fall08articles/boston-showcases.html

Dragićević, S. (2004). The Potential of Web-based GIS. Journal of Geographical Systems, 6(2), 79-81. doi: 10.1007/s10109-004-0133-4

Dubayah, R. \& Rich P.M. (1996). GIS-Based Solar Radiation Modeling. In M.J. Goodchild, L.T. Steyaert, B.O. Parks, C. Johnson, D. Maidment, M. Crane, \& S. Glendinning (Eds.), GIS and Environmental Modeling: Progress and Research Issues (pp. 129-134).

Elaksher, A. F. \& Bethel, J. S. (2002). Reconstructing 3D Buildings from LiDAR Data. Proceedings of the International Society for Photogrammetry and Remote Sensing Conference.

ESRI (2007). Working with ArcGIS Spatial Analyst. Redlands, CA: ESRI.

ESRI (2009). Introduction to ArcGIS Server. Redlands, CA: ESRI.

ESRI (2010a). An Overview of the Solar Radiation Tools. Retrieved May 7, 2010 from http://webhelp.esri.com/arcgisdesktop/9.3/index.cfm?TopicName=An_overview of_the_Solar_Radiation_tools

ESRI (2010b). Solar Radiation Graphics. Retrieved May 7, 2010 from 
http://webhelp.esri.com/arcgisdesktop/9.3/index.cfm?TopicName=Solar_Radiatio $\underline{\text { n_Graphics }}$

ESRI (2010c). Managing LiDAR Data in ArcGIS [Virtual Campus Course]. Retrieved June 09, 2010.

ESRI (2010d). Calculating Solar Radiation. Retrieved May 5, 2010 from http://webhelp.esri.com/arcgisdesktop/9.3/index.cfm?TopicName=Calculating_so $\underline{\text { lar_radiation }}$

ESRI (2010e). Understanding Solar Radiation Analysis. Retrieved June 14, 2010 from http://webhelp.esri.com/arcgisdesktop/9.3/index.cfm?id=6052\&pid=6051\&topicn $\underline{\text { ame}}=$ Understanding_solar_radiation_analysis

ESRI (2010f). Points Solar Radiation. Retrieved April 20, 2010 from http://webhelp.esri.com/arcgisdesktop/9.3/index.cfm?TopicName=Points_Solar_ $\underline{\text { Radiation }}$

ESRI (2010g). ArcGIS JavaScript API Overview. Retrieved June 20, 2010 from http://resources.esri.com/help/9.3/arcgisserver/apis/javascript/arcgis/help/jshelp_s $\underline{\text { tart.htm }}$

ESRI (2010h). Identify Features on a Map. Retrieved June 21, 2010 from http://resources.esri.com/help/9.3/arcgisserver/apis/javascript/arcgis/help/jssample s_start.htm

Fielding, R. T. (2000). Architectural Styles and the Design of Network-based Software Architectures (Doctoral dissertation, University of California, Irvine). Retrieved from http://www.ics.uci.edu/ fielding/pubs/dissertation/top.htm

Fu, P. \& Rich, P. (1999). Design and Implementation of the Solar Analyst: an Arc View Extension for Modeling Solar Radiation at Landscape Scales. Proceedings of the Nineteenth Annual ESRI User Conference. Retrieved from http://proceedings.esri.com/library/userconf/proc99/proceed/papers/pap867/p867. $\underline{\mathrm{htm}}$

Fu, P. \& Rich, P. (2002). A Geometric Solar Radiation Model with Applications in Agriculture and Forestry. Computers and Electronics in Agriculture, 37(2002), 25-35.

Kandt, A., Dean, J., Burman, K., \& Helm, C. (2009). Solar 2009: Web-based Solar Photovoltaic Mapping Tools. Retrieved from ESRI ArcGIS Resource Centers website: http://resources.esri.com/arcgisserver/apis/flex/index.cfm?fa=codeGalleryDetails \&scriptId $=16110$ 
Lillesand, T. M., Kiefer, R. W., \& Chipman, J. W. (2008). Microwave and LiDAR Sensing. Remote Sensing and Image Interpretation $-6^{\text {th }}$ ed. (pp. 626-726). John Wiley \& Sons, Inc.

Lloyd, C. D. \& Atkinson, P. M. (2006). Deriving Ground Surface Digital Elevation Models from LiDAR Data with Geostatistics. International Journal of Geographical Information Science, 20(5), 535-563.

Los Angeles County. (2009). Los Angeles County Solar Map. Retrieved October 17, 2009 from http://lacounty.solarmap.org/

Ma, R. (2005). DEM Generation and Building Detection from Lidar Data. Photogrammetric Engineering \& Remote Sensing, 71(7), 847-854.

Peng, Z, \& Tsou, M. (2003). GIS, Internet GIS, and Distributed GIServices. Internet GIS - Distributed Geographic Information Services for the Internet and Wireless Networks. (pp. 1-35). Hoboken, NJ: John Wiley \& Sons, Inc.

Podobnikar, T., Oštir, K., \& Zakšek, L. (2006). Influence of Data Quality on Solar Radiation Modeling. In M. Campagna (Ed.), GIS for Sustainable Development. (pp. 417-430). Boca Raton, FL: CRC Press.

Rottensteiner, F. (2003). Automatic Generation of High-Quality Building Models from Lidar Data. IEEE Computer Graphics and Applications, 23(6), 42-50. doi: 10.1109/MCG.2003.1242381

Ruiz-Arias, J. A., Tovar-Pescador, J., Pozo-Vázquez, D., \& Alsamamra, H. (2009). A Comparative Analysis of DEM-based Models to Estimate the Solar Radiation in Mountainous Terrain. International Journal of Geographical Information Science, 23(7- 8), 1049-1076.

Taylor, G., Li, J., Kidner, D., Brunsdon, C., \& Ware, M. (2007). Modelling and Prediction of GPS Availability with Digital Photogrammetry and LiDAR. International Journal of Geographical Information Science, 21(1-2), 1-20.

Tomlinson, R. (2007). Choose a Logical Data Model. Thinking about GIS: Geographic Information System Planning for Manager $-3^{\text {rd }}$ ed. (pp. 93-94). Redlands, CA: ESRI Press.

United Nations. (2009). World Population Prospects: The 2008 Revision Population Database. Retrieved October 19, 2009 from http://esa.un.org/unpp/index.asp 



\section{Appendix A. Web GIS Application Code}

The following programming code was used to develop the University of Redlands Rooftop Solar Potential application using JavaScript API. All GIS functions and user interface were created by this code that is available in an htm file.

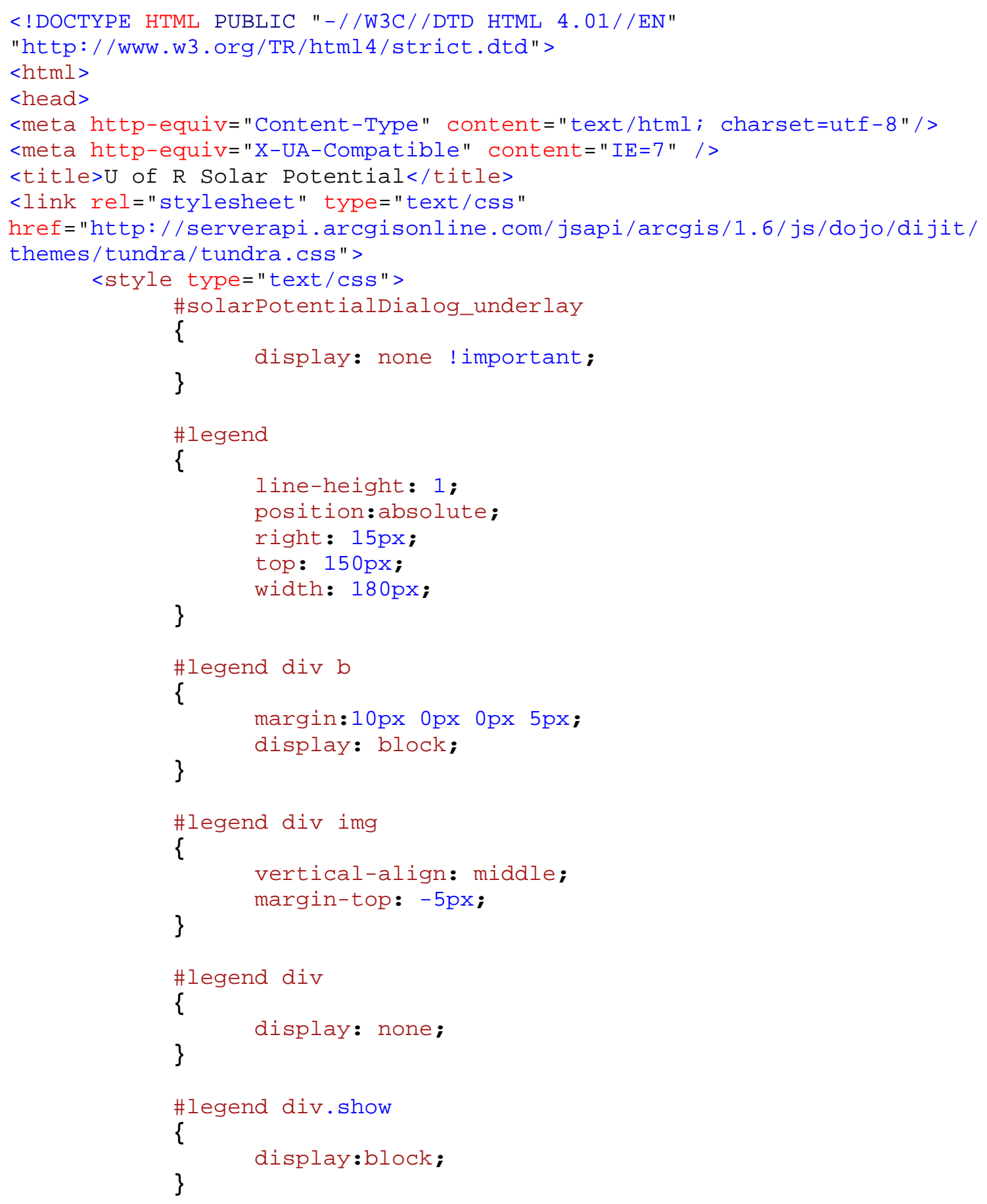




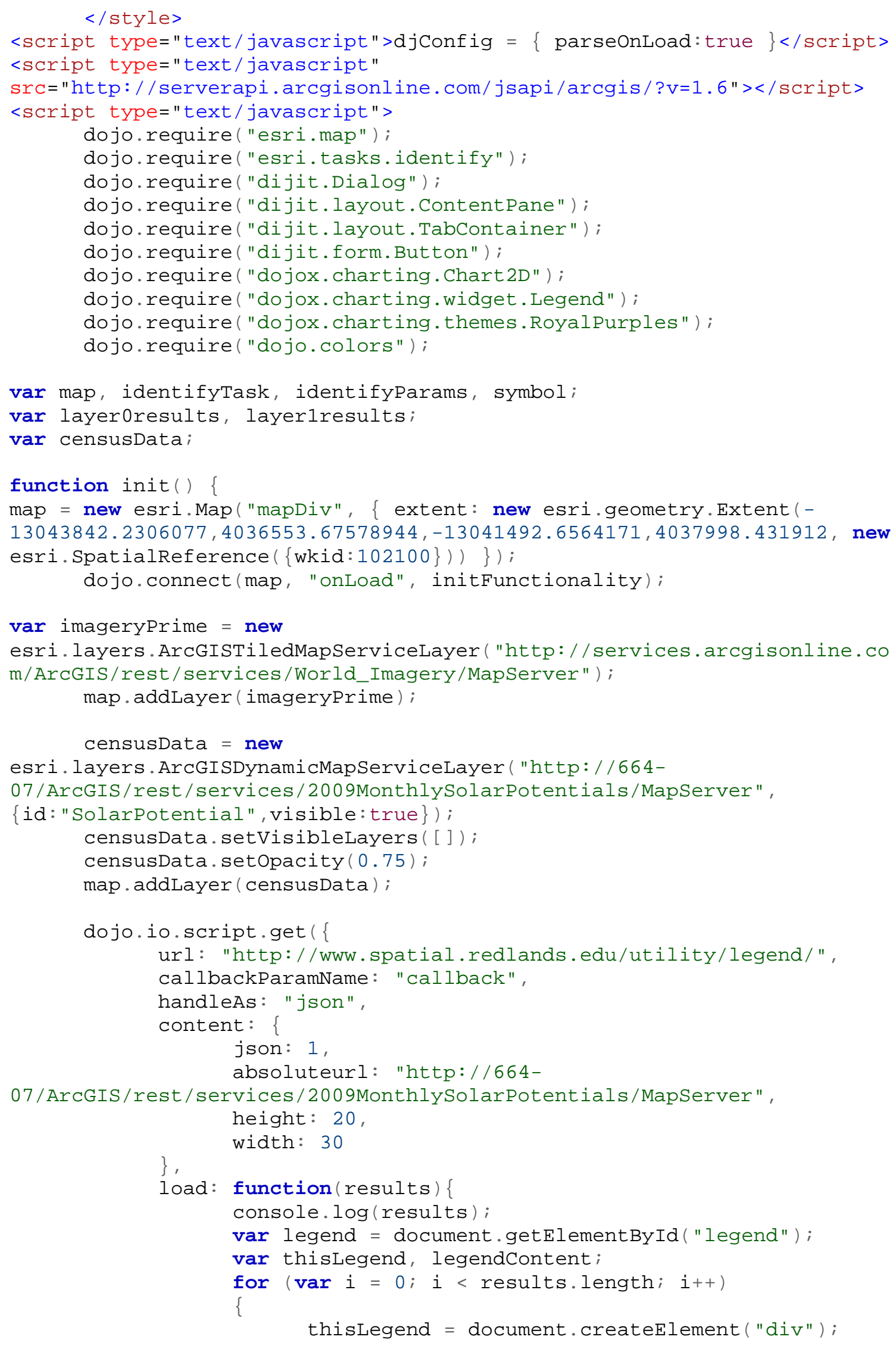




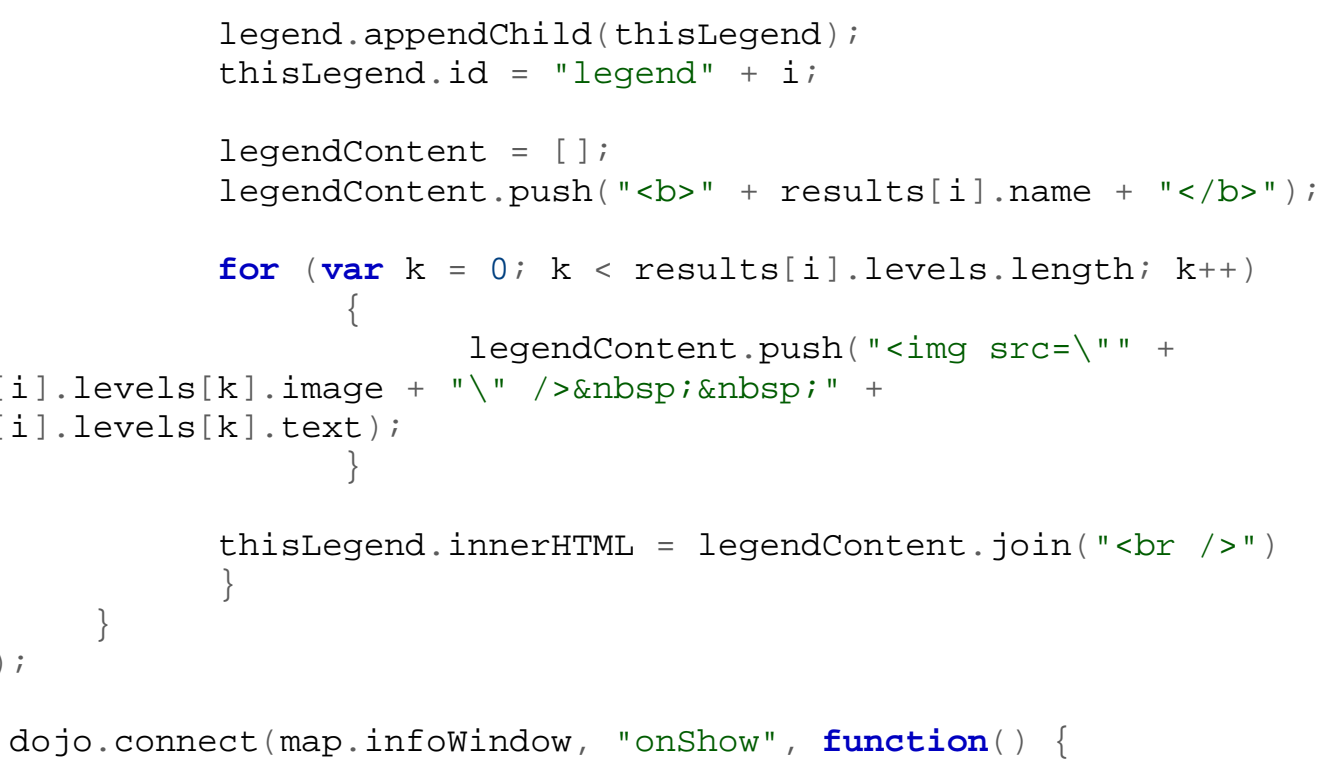


symbol = new

esri.symbol.SimpleFillsymbol(esri.symbol.SimpleFillsymbol.STYLE_SOLID, new

esri.symbol.SimpleLineSymbol(esri.symbol.SimpleLineSymbol.STYLE_SOLID, new dojo.Color $([255,0,0]), 2)$, new dojo.Color $([255,255,0,0.5]))$;

\}

function doIdentify(evt) \{

map.graphics.clear ( );

identifyParams.geometry = evt.mapPoint;

identifyParams. mapExtent = map.extent;

identifyTask. execute(identifyParams, function(idResults) \{

addToMap(idResults, evt); \});

\}

var solarchart;

function addToMap(idResults, evt) \{

if (idResults. length $<1$ )

return;

layeroresults $=\{$ displayFieldName: null, features: [] $\}$;

layer1results $=\{$ displayFieldName: null, features: []$\}$;

for (var $i=0, i l=i d R e s u l t s . l e n g t h ; i<i l ; i++)\{$

var idResult = idResults[i];

if (idResult. layerId $===0$ ) \{

if (! layeroresults displayFieldName)

\{layeroresults.displayFieldName = idResult.displayFieldName ;

layeroresults. features.push(idResult . feature);

\} else if (idResult. layerId $===1$ ) \{

if (!layer1results displayFieldName)

\{layer1results.displayFieldName = idResult.displayFieldName\}; \} layer1results. features.push(idResult . feature);

\}

console. $\log ($ layer1results);

showFeature (layer1results . features $[\odot]$ );

var dialog = dijit.byId("solarPotentialDialog");

if (!dialog.open)

dialog. show( );

dialog. oncancel $=$ function( )

\{

dialog.hide ( );

\}

map.graphics.clear ( );

dialog.domNode.style.left = "50px";

dijit.byId("tabs"). selectChild(dijit. byId("layer@Tab"));

document.getElementById("renderedChart"). innerHTML = " "; solarChart = new dojox. charting.Chart2D("renderedchart"); 


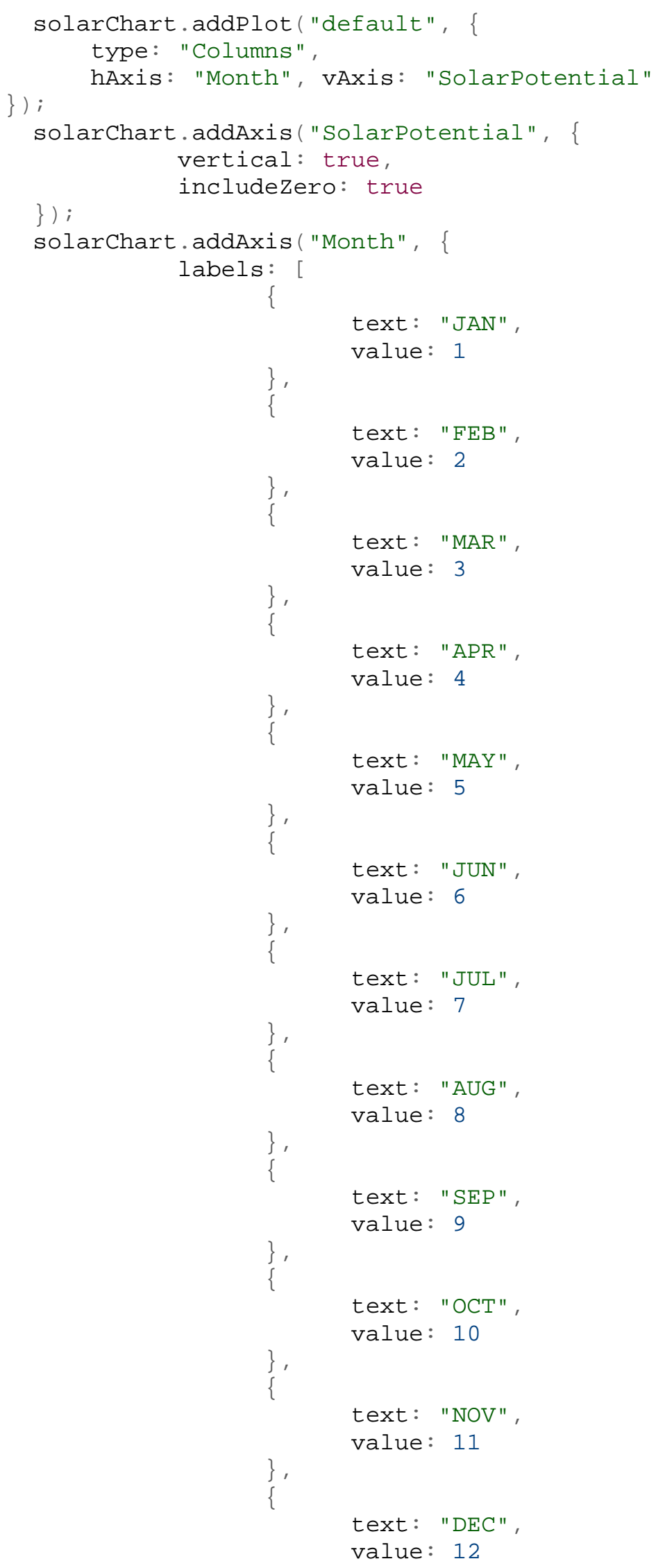



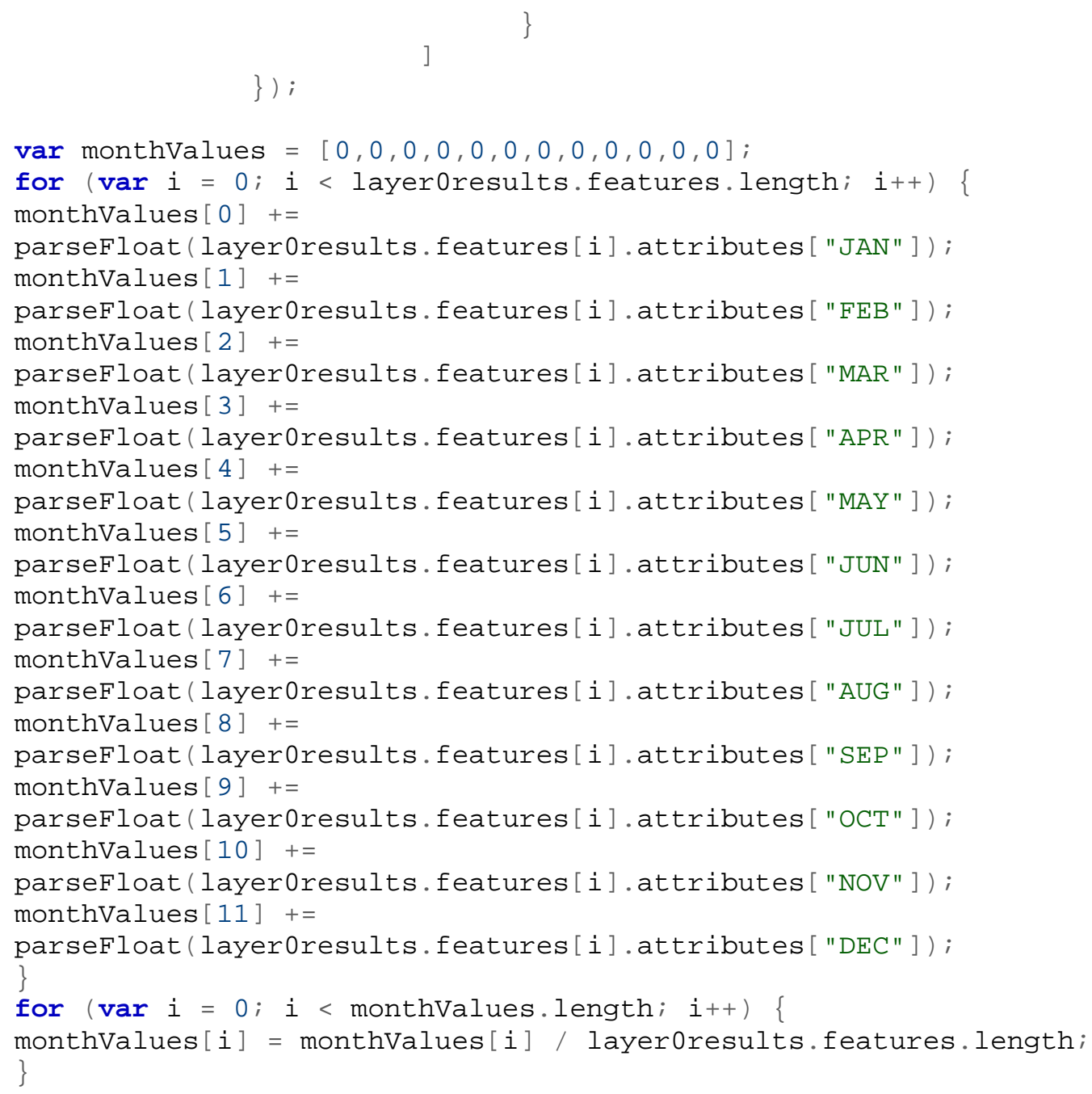

function layerTabcontent(layerResults, layerName) \{

var content = "";

switch (layerName) \{ 


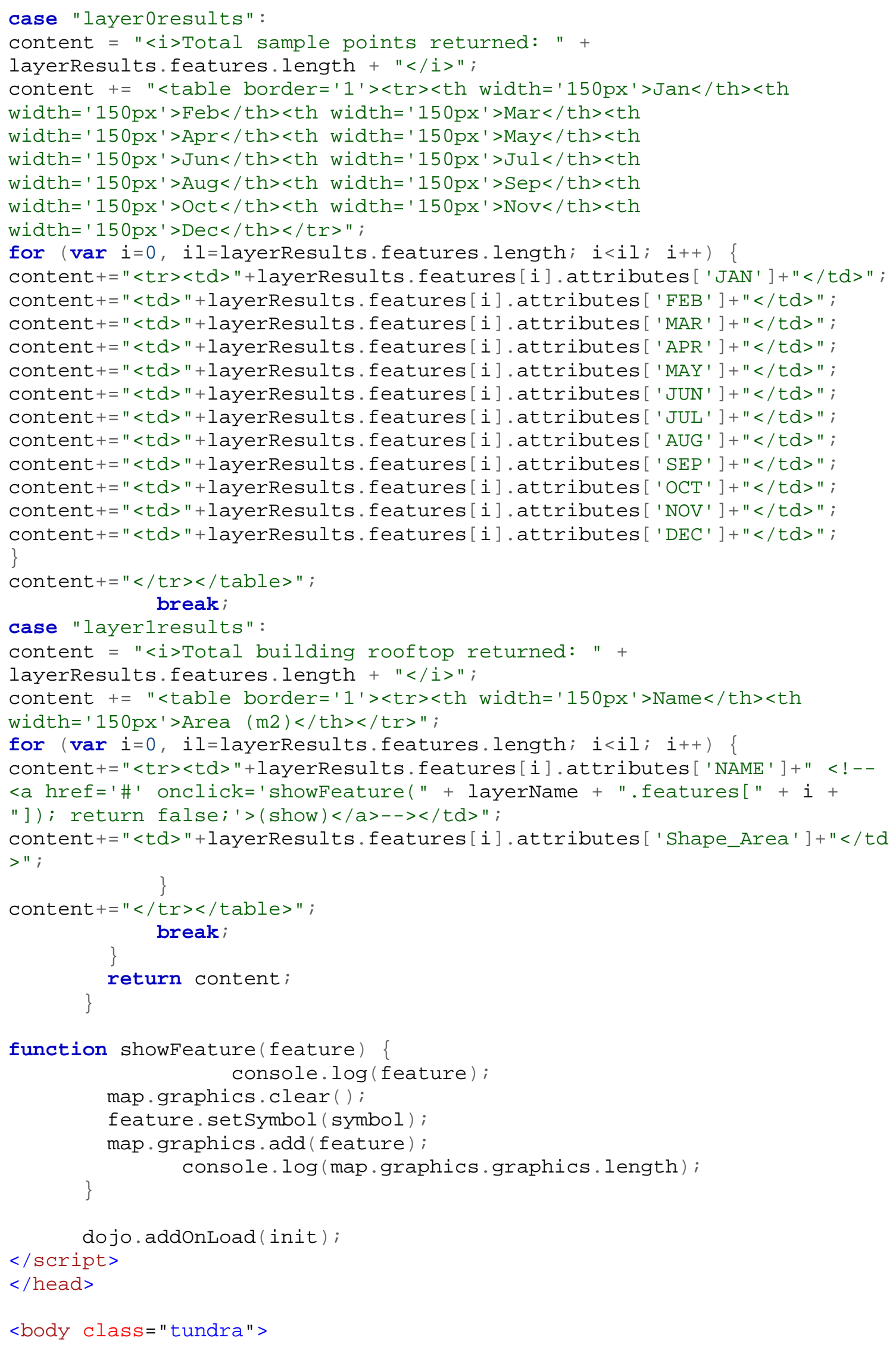


<body bgcolor="\#FAF8CC">

$<$ div id="header">

$<$ a id="title" target="_blank" title="University of Redlands Rooftop

Solar Potential Viewer" href="http://wWw.ci.redlands.ca.us/"><h1

align="center">University of Redlands Rooftop Solar Potential $</$ h 1$\rangle\langle/$ a $>$ $</$ div $>$

$<$ div align="center" $><p$ style="font-size:20px">click the map to identify a rooftop and display the building name and the 2009 solar potential information. $<\mathrm{br} /></ \mathrm{p}>$

$</$ div $>$

$<$ div align="center" $><p$ style="font-size:15px"><span id="layer_list"> <input type='radio' name="layer" class='list_item' id="layer@" value $=" \odot$ " onclick='updateLayerVisibility ()$; ' /><$ label

for $=$ "layer $\odot ">$ JAN $</$ label $>$ \&nbsp; \&nbsp;

<input type='radio' name="layer" class='list_item' id="layer1"

value="1" onclick=' updateLayerVisibility ( ); ' $/><$ label

for ="layer1">FEB </label>\&nbsp;\&nbsp;

<input type='radio' name="layer" class='list_item' id="layer2"

value="2" onclick='updateLayerVisibility ( ); '/><label

for="layer $2 ">$ MAR $</$ label $>\&$ nbsp; \&nbsp;

<input type='radio' name="layer" class='list_item' id="layer3"

value="3" onclick='updateLayerVisibility ( ); '/><label

for ="layer 3 ">APR</label>\&nbsp; \&nbsp;

<input type='radio' name="layer" class='list_item' id="layer4"

value="4" onclick='updateLayerVisibility ( ); '/><label

for $=$ "layer 4" $>$ MAY </label $>$ \&nbsp; \&nbsp;

<input type='radio' name="layer" class='list_item' id="layer5"

value $=$ "5" onclick='updateLayerVisibility ( ); '/ $><$ label

for="layer5">JUN</label>\&nbsp;\&nbsp;

<input type='radio' name="layer" class='list_item' id="layer6"

value="6" onclick='updateLayerVisibility ( ); '/><label

for ="layer6" > JUL </label>\&nbsp; \&nbsp;

$<$ input type='radio' name="layer" class='list_item' id="layer7"

value $=" 7$ " onclick='updateLayerVisibility ()$; ' /><$ label

for ="layer7">AUG</label>\&nbsp; \&nbsp;

<input type='radio' name="layer" class='list_item' id="layer8"

value="8" onclick='updateLayerVisibility ( ); '/><label

for $=$ "layer 8 " $>$ SEP $</$ label $>$ \&nbsp; \&nbsp;

<input type='radio' name="layer" class='list_item' id="layer9"

value="9" onclick='updateLayerVisibility ( ); '/><label

for ="layer9">0CT</label>\&nbsp; \&nbsp;

<input type='radio' name="layer" class='list_item' id="layer10"

value $=" 10$ " onclick='updateLayerVisibility ()$; ' /><$ label

for $=$ "layer10">NOV</label>\&nbsp; \&nbsp;

<input type='radio' name="layer" class='list_item' id="layer11"

value="11" onclick='updateLayerVisibility ()$; ' /><$ label

for="layer11">DEC</label>\&nbsp; \&nbsp;

<input type='radio' name="layer" class='list_item' id="layer12"

value="12" onclick='updateLayerVisibility ( ); '/><label

for="layer12">0UTLINES</label>\&nbsp; \&nbsp;

<input checked="checked" type='radio' name="layer" class='list_item' id="layer13" value="" onclick='updateLayerVisibility();'/><label

for $="$ layer 13 " $>$ NONE $</$ label $>$ \&nbsp; \&nbsp $;</ p></$ span $></$ div $>$

$<$ div $>$

<div id="mapDiv" style=" margin: $\odot$ auto; width:1000px; height:525px;

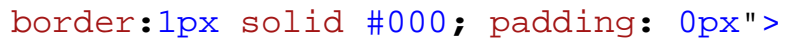




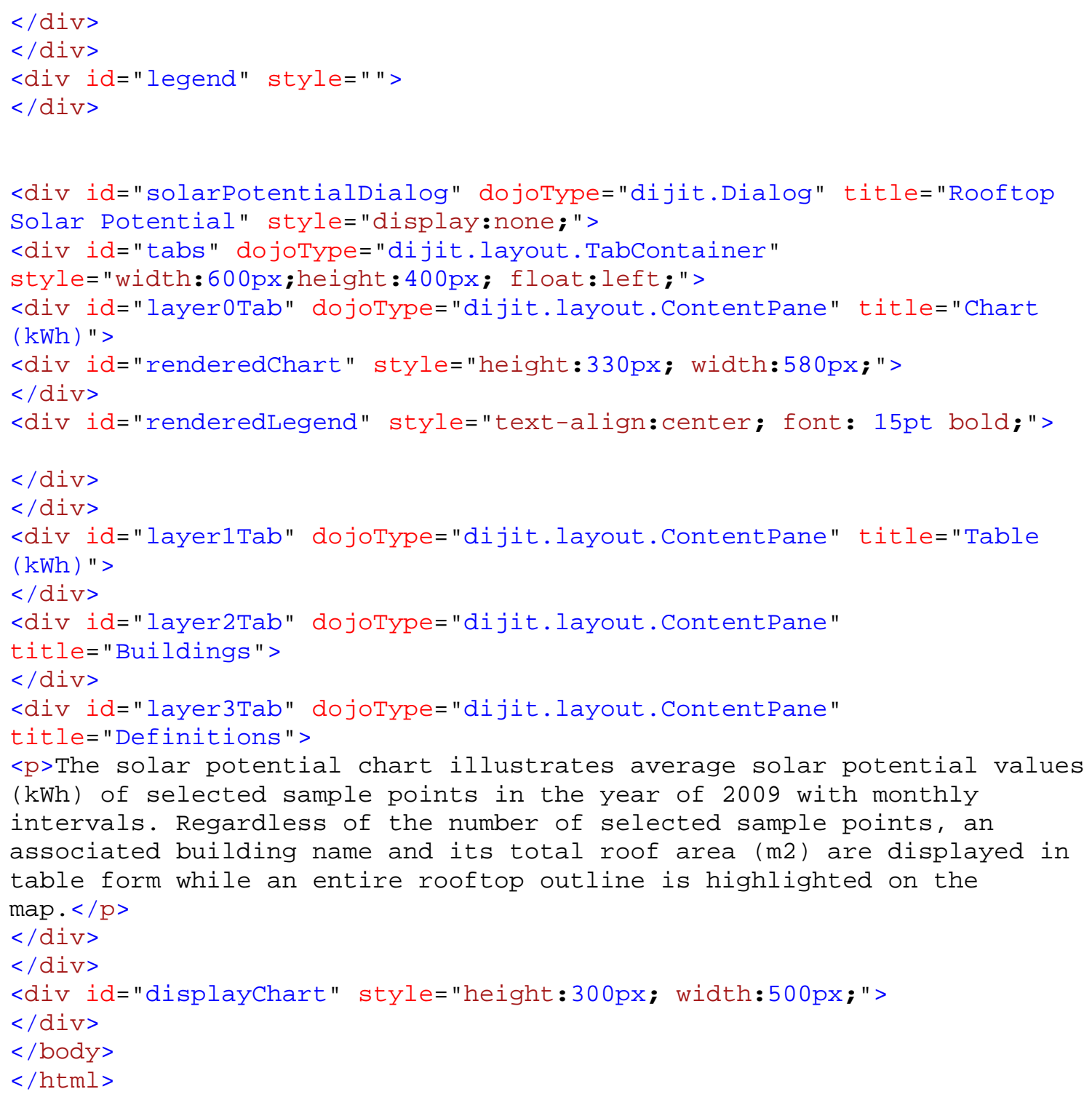

